\title{
On the development of a multi-gate pulsed Doppler system with serial data-processing
}

Citation for published version (APA):

Hoeks, A. P. G. (1982). On the development of a multi-gate pulsed Doppler system with serial dataprocessing. [Doctoral Thesis, Maastricht University]. Rijksuniversiteit Limburg. https://doi.org/10.26481/dis.19820305ah

Document status and date:

Published: 01/01/1982

DOI:

10.26481/dis.19820305ah

Document Version:

Publisher's PDF, also known as Version of record

\section{Please check the document version of this publication:}

- A submitted manuscript is the version of the article upon submission and before peer-review. There can be important differences between the submitted version and the official published version of record. People interested in the research are advised to contact the author for the final version of the publication, or visit the DOI to the publisher's website.

- The final author version and the galley proof are versions of the publication after peer review.

- The final published version features the final layout of the paper including the volume, issue and page numbers.

Link to publication

\footnotetext{
General rights rights.

- You may freely distribute the URL identifying the publication in the public portal. please follow below link for the End User Agreement:

www.umlib.nl/taverne-license

Take down policy

If you believe that this document breaches copyright please contact us at:

repository@maastrichtuniversity.nl

providing details and we will investigate your claim.
}

Copyright and moral rights for the publications made accessible in the public portal are retained by the authors and/or other copyright owners and it is a condition of accessing publications that users recognise and abide by the legal requirements associated with these

- Users may download and print one copy of any publication from the public portal for the purpose of private study or research.

- You may not further distribute the material or use it for any profit-making activity or commercial gain

If the publication is distributed under the terms of Article $25 \mathrm{fa}$ of the Dutch Copyright Act, indicated by the "Taverne" license above, 


\section{ON THE DEVELOPMENT OF A MULTI-GATE PULSED DOPPLER SYSTEM WITH SERIAL DATA-PROCESSING}

PROEFSCHRIFT

ter verkrijging van de graad van doctor in de geneeskunde aan de Rijksuniversiteit Limburg te Maastricht op gezag van de Rector Magnificus Prof. Dr. H.C. Hemker volgens besluit van het College van Dekanen in het openbaar te verc̄edigen in de aula va de universiteit op vrijdag 5 mart 1982 des namiddags om 4 uur

door

\section{ARNOLD P.G. HOEKS}

geboren te Bergeijk 
PROMOTORES: Prof. Dr. R.S. Reneman, University of Limburg, Maastricht, the Netherlands

Prof. Dr. N. Bom, Erasmus University, Rotterdam, the Netherlands

REFERENTEN: Prof. Dr. J. Mol, Univerisity of Limburg, Maastricht, the Netherlands Prof. Dr. B.A.J. Angelsen, Norwagian Institute of Technology, Trondheim, Noorwegen

This work was supported by:

- the Foundation for Medical Reseach Eungo which is subsidized by the Dutch organization for the advancement of pure research (ZwO)

- the Dutch Heart Foundation

Het verschijnen van dit proefschrift werl mede mogelijk gemaakt door steun van de Nederlandse Hartstichting.

$\begin{array}{ll}\text { Typewerk : } & \text { R.M. Borgman-Hanssen } \\ \text { Tekeningen: } & \text { H. Loete } \\ \text { Omslag : } & \text { E. Daniels, F. Smeets } \\ \text { Drukkerij: } & \text { Econooom, Beek (L) }\end{array}$


AAN MIJN OUDERS 
CONTENTS

CONTENTS

1. INTRODUCTION

2. ULTRASOUND DOPPLER SYSTEMS

2.I Basic principles of ultrasound II

2.2 The ultrasound beam 12

2.3 Doppler principle 15

$\begin{array}{lll}2.4 & \text { Doppler systems } & 17\end{array}$

3. CW-DOPPLER SYSTEMS

3.1 Introduction 19

3.2 Receiver, Demodulator 19

3.3 AuAio-evaluation $\quad 22$

3.4 Frequency analysis of the Doppler signal 23

3.5 Detection of average velocity 25

3 . 6 Discussion 31

4. PULSED DOPPLER SYSTEMS

4.1 Basic concepts 33

4.2 Transducer $\quad 35$

4.3 Receiver 36

4.4 Phase detector 38

4.5 Sample-volume 39

4.6 Doppler bandpass filter 41

4.7 Detection of average velocity 42

4.8 Discussion 44

5. MULTI-GATE PULSED DOPPLER SYSTEM

5.1 Introduction $\quad 45$

5.2 Sampled data filters 45

5.3 The aynamic range of the digital filter 51

5.4 Timing considerations for the digital filtar 54

5.5 Datection of average frequency 58 
6

5.6 Detection of signal amplitude 62

5.7 Detection of relative change in vessel diameter 65

5.8 Ultrasonographic blood flow imaging 70

5.9 System description 71

5.10 Real-time display of time-dependent velocity 77 profiles

5.11 Discussion 33

6. IMPROVEMENT OF THE FREQUENCY OETECTOR CHARACTERISTICS

$\begin{array}{lll}6.1 & \text { Introduction } & 37\end{array}$

6.2 Frequency aliasing 90

6.3 Doppler signal simulation $\quad 92$

6.4 Evaluation of the time-discrete directional 94 zero crossing detector

6.5 The instantaneous frequency 98

6.6 The probability density function of the 100 instantaneous Erequency

6.7 Relative error and standard deviation of the 107 time average of the instantaneous frequency

6.8 Tracking behaviour of the correction mechanism 112

6.9 Hardware realization of the instantaneous 116 Erequency detector

7. CLINICAL APPLICATIONS 125

8. Conclusion 137

SUMMARY

SAMENVA T'TING

REFERENCES

NAWOORD 


\section{INTRODUCTION}

The spatial and temporal behaviour of the blood flow through arteries is affected by the condition of these arteries. Local narrowing of an artery due to a stenosis will cause elevated blood flow velocities within and just distal to the stenosis (Blackshear, 1980; Khalifa, 1981). If dilatation of the vascular bed or of the smaller arteries (arterioles) is unable to compensate for the increased flow resistance in the stenosis the blood supply to the tissues distal to the stenosis will decrease. Plaque-formation introduces an additional hazara because plaques are believed to be a source of emboli causing for example transient ischemic attacks. Unlike stenoses plaques (small local irregularities of the vesse wall) will hardly affect volume flow but will mainly disturb the flow pattern locally (Sandmann, 1978; Wille, 1981) and, therefore, the velocity distribution along the cross-section of the artery (i.e. the velocity profile).

In the past several methods have been developed to detect the presence of stenoses or occlusions in arteries. Some of these methods involve penetration of the vessel wall to inject a contrast medium (angiography) or a catheter tip (e.g. anemometers). Angiography is widely used in the clinic and is up to now considered as the gold standard for the investigation of the arterial tree. However, this method does not provide information about the dynamic behaviour of blood flow i.e. the blood velocity as function of time and place. The anemometer gives this information but it is less suitable for clinical applications because of the time involved in exploring a segment of an artery. That is why it is only used to monitor blood flow velocity. It shares this application with the electromagnetic flowmeter which requires positioning of a probe around the artery. Because the probe is selected so that its diameter matches the diameter of the artery the electromagnetic flowmeter does not only provide accurate information about the 
blood flow velocity averaged over the cross-section of the artery but also about the volume flow, both as instantaneous function of time.

Because of their invasiveness the above mentioned methods are unsuitable for screening of diseased arteries. Moreover, they are too global or too cumbersome for the detection of plaque formation.

The well-known Doppler systems (Reneman, 1977; Reneman, 1981b) based on the emission and the subsequent analysis of the backscattered sound waves, have the potency to detect plaque formation and are already widely used to detect stenosed arteries. They can be employed transcutaneously and are a-traumatic, at least at the intensity levels at which they are normally operated. The trancutaneous assessment of blood flow velocities in major arteries with Doppler systems has the additional advantage that the method does not interfere with the processes under investigation. Unlike catheter-tip devices it does not cause a disturbance of the blood velocity distribution along the cross-section of the artery.

In the past decades Doppler systems have evolved from simple, general purpose systems to dedicated and highly sophisticated equipment. Part of this development can be contributed to the need to obtain more reliable information. The early systems of the continuous wave type were unable to detect the direction of the blood flow. Since the availability of directional systems it became apparent that the analyzed backscattered signals were contaminated by Doppler signals originating from outside the region of interest. The incorporation of some dedicated processing equipment, resulting in seperate processing of the Doppler signals induced by forward and backward flow, will reveal this unwanted situation at an early stage and will avoid misinterpretation of the recorded velocity signals. The introduction of spectral analysis enabled investigators to extract more detailed information from the Doppler signals. 
Despite these improvements $\mathrm{CW}$-Doppler systems cannot provide accurate and detailed information about the velocity distribution along the cross-section of an artery as function of time. pulsed Doppler systems, emitting repeatedly short bursts of ultrasound and subsequently analyzing the received signals, yield the possibility to focus on a small sample volume that can be located within the artery of interest. In single-gate pulsed Doppler systems the problem of positioning the sample volume properly is overcome either using large sample volumes (causing a loss in detail) or combining the system with an ultrasound echo system providing a two-dimentional image of the underlying tissues. Another approach is the application of a multi-gate pulsed Doppler system enabling the simultaneous investigation of blood flow velocities in a number of adjacent sample volumes. If a large number of sample volumes is employed the problem of locating a vessel is greatly reduced and small sample volumes can be taken. Since the assessment of the blood flow velocity in the adjacent sample volumes is performed simultaneously, the velocity distribution along the cross-section of the artery under investigation as function of time can be determined. Multi-gate pulsed Doppler systems will henceforth provide detailed information in realtime. Since the presence of stenoses or (calcified) plaques will affect the temporal and spatial behaviour of the velocity profile, multi-gate pulsed Doppler systems offer therefore some advantages over $\mathrm{CW}$-Doppler systems. However, the introduction of multi-gate systems in the clinic or even in research laboratories is impeded by the complicated design of such a system.

The conventional approach to construct a multi-gate system is to start with a single-gate pulsed Doppler system and to duplicate the processing circuitry for each additonal gate. This approach, however, makes the apparatus sensitive to noise because the processing circuitry for different sample volumes will interfere, although crosstalk can be reduced by proper shielding. Moreover, all the processing channels should have the same characteristics and hence require careful tuning. That 
is why the number of processing channels is generally limited to the lowest possible number which means that the sample volumes are just covering an artery with a given diameter. To circumvent this problem, some years ago we started with the development of a multi-gate pulsed Doppler system with serial rather than parallel data-processing. In the serial approach the signals originating from adjacent sample volumes are analyzed by a single processing circuit via time-multiplexing. In this way the required circuitry will be almost independent of the number of employed sample volumes and tuning is reduced to a minimum, i.e. the tuning required for a single gate system. Since serial processing eliminates some of the problems encountered in pulsed Doppler systems with parallel data processing, time can be dedicated to the development of some additional features. With pulsed Doppler systems one can not only investigate the blood flow velocity as function of time and place but also the movements of the arterial walls. The simultaneous assessment of the relative change in vessel diameter and the velocity profile during a cardiac cycle may provide additional evidence for the existence of vessel wall abnormalities.

This dissertation deals with the development of a multi-gate pulsed Doppler system with serial data processing for transcutaneous investigation of blood flow velocities and vessel wall movement of superficial arteries (down to a depth of about 40 $\mathrm{mm})$. To make the reader acquaintance with the underlying assumptions, the concepts of the employed signal analysis technique will be discussed in detail. That is why attention is paid to the design of a continuous wave Doppler system. The applicability of the system will be demonstrated with some case studies. 


\section{ULTRASOUND DOPPLER SYSTEMS}

\subsection{BASIC PRINCIPLES OF ULTRASOUND}

Whe relation between frequency $f$, wavelength $\lambda$ and speed of sound $c$ is given by the relation: $c=\lambda * f$. For a given speed of propagation there exists therefore a reciprocal relation between $\lambda$ and $f$ : increasing the frequency will reduce the wavelength. The speed of sound in water and in tissues is in the order of $1550 \mathrm{~m} / \mathrm{s}$. Unless otherwise indicated we will use in the calculations $\mathrm{c}=1500 \mathrm{~m} / \mathrm{s}$.

The acoustic impedance $z$ (Wells, 1969) gives the relation between pressure variations induced by the sound waves and the velocity of the particles moving due to these pressure variations. The impedance $z$ can be expressed in terms of the density $\rho$ of the medium and the speed of sound in that medium and equals $Z=\rho * c$. For water the acoustic impedance therefore will be in the order of $1.5 * 10^{6} \mathrm{~kg} \mathrm{~m}^{2} \mathrm{~s}^{-1}$. At the interface of mediums with a different acoustic impedance the sound waves will be partly reflected. The reflection coefficient, indicating the fraction of energy that is reflected, can be expressed as:

$$
\rho_{R}=\left(z_{2}-z_{1}\right)^{2} /\left(z_{2}+z_{l}\right)^{2}
$$

where $z_{1}$ and $z_{2}$ is the acoustic impedance in medium 1 and 2 , respectively. In this relation it is assumed that the interface is perpendicular to the direction of propagation of the sound waves. The non reflected sound energy will travel into the second medium. Sound waves will be reflected under the same angle as the angle of incidence. If the dimension of the interface becomes small compared to the wavelength of the impinging sound, as in the case of blood particles, the sound wave will not be reflected in a particular direction but will be radiated in all directions (scattering). The scattering behaviour of a conglomeration of particles suspended in a medium is $r$ ather complicated (Angelsen, 1980a). Increasing the sound frequency 
(and thereby reducing the wavelength) will increase the intensity of the sound scattered back by the particles. For the commonly applied sound frequencies the scattering power of blood particles is proportional to the fourth power of the sound frequency (Shung, 1976; Angelsen, 1980a). It would be therefore advisable to select a sound frequency as high as possible to get more power back from the particles. On the other hand as sound travels through a medium it will be absorbed by that medium. The absorption is expressed as the fraction of the sound energy that is absorbed as the sound waves cover a given distance. This absorption is for the commonly employed sound frequency in tissues proportional to the square of the sound frequency (Wells, 1969). The optimal frequency in terms of the acoustic power received from blood particles will be therefore a function of depth of investigation as well. Increasing the depth of investigation requires reduction of the sound frequency.

\subsection{THE ULTRASOUND BEAM}

The sound is emitted into the tissues by an electro-acoustical transducer converting electric into acoustical energy. The transducer is made of a piezo-electric material for Doppler applications shaped as a disc. The thickness of the disc determines the optimal frequency for the transducer. For this frequency the thickness of the disc equals half the wavelength of the sound in the material and the conversion of electric into acoustic energy is most efficient. This can be illustrated with the impedance characteristic of a commercially available CWtransducer (fig. 2.1). The transducer including the cable attains the lowest impedance at the resonance frequency of 3.8 $\mathrm{MHz}$. For other frequencies the transducer will be less efficient.

The diameter of the transducer disc affects the way in which the sound is radiated. A flat wave front can only be generated 


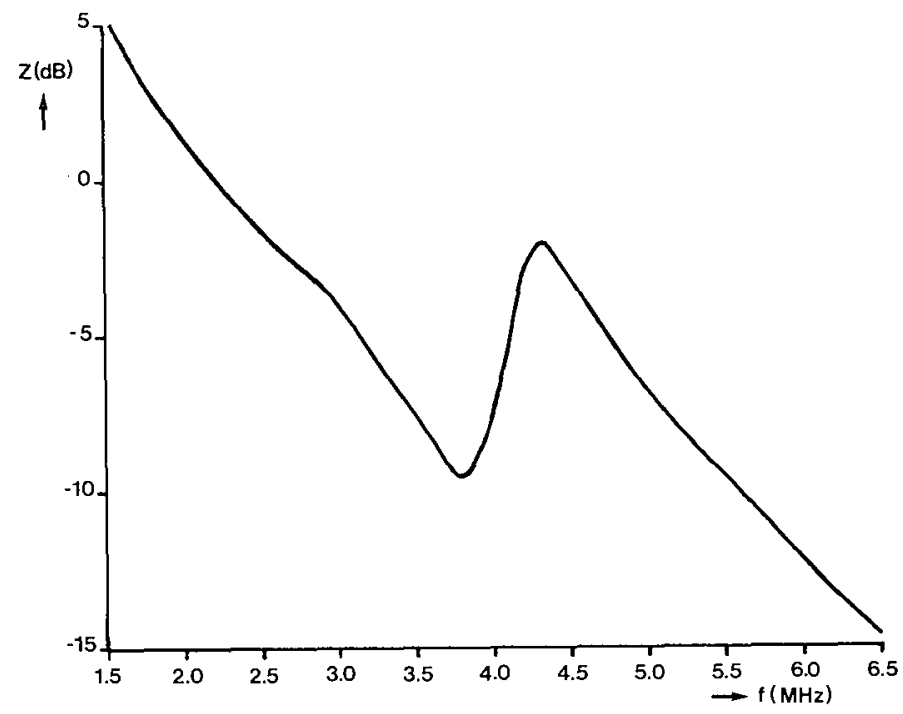

Fig. 2.1 : Impedance characteristic of a CW-transducer including the cable. The transducer attains its lowest impedance at $3.8 \mathrm{MHz}$ (resonance frequency).

if the diameter is large as compared to the wavelength, but this results in a wide sound beam. A generalized shape of the emitted sound beam is depicted in fig. 2.2. Far away from the transducer in terms of wavelengths the sound beam diverges (far field) while close to the transducer the diameter of the beam is in the order of the diameter $D$ of the transducer (near field). The transition from near field to far field is at a distance of $\mathrm{D}^{2} / 4 \lambda$. A narrow beam over a long range can only be

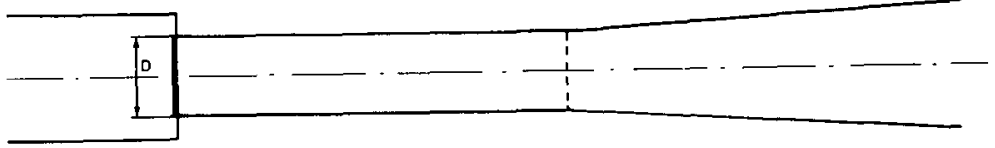

Fig. 2.2 : Generalized shape of the ultrasound beam. The beam starts to diverge at a range of $D^{2} / 4 \lambda$.

obtained by employing a high emission frequency and a small transducer diameter. This restriction is less critical if one limits the application to superficial investigations. To obtain 
a beam width of $6 \mathrm{~mm}$ down to a distance of $60 \mathrm{~mm}$ demands an emission frequency in the order of $10 \mathrm{MHz}$ for a $6 \mathrm{~mm}$ disc. If this frequency is not feasible because of signal level considerations, one can only increase the diameter of the transducer to maintain the same point of transition at the expense of a wider beam in the near field. A lower emission frequency will improve the signal level but will adversely affect the lateral resolution of the system. However, a local narrow beam can be obtained by focussing the transducer using either an acoustic lens or a curved transducer.

The boundaries of the sound beam are not as distinct as depicted in fig. 2.2. Most of the sound energy will be concentrated in the middle of the beam. The energy level will gradually fade out when moving away from the axis of the beam. This is illustrated in fig. 2.3 where the sound pressure distribution of a CW-transducer is presented. Since the transaucer is

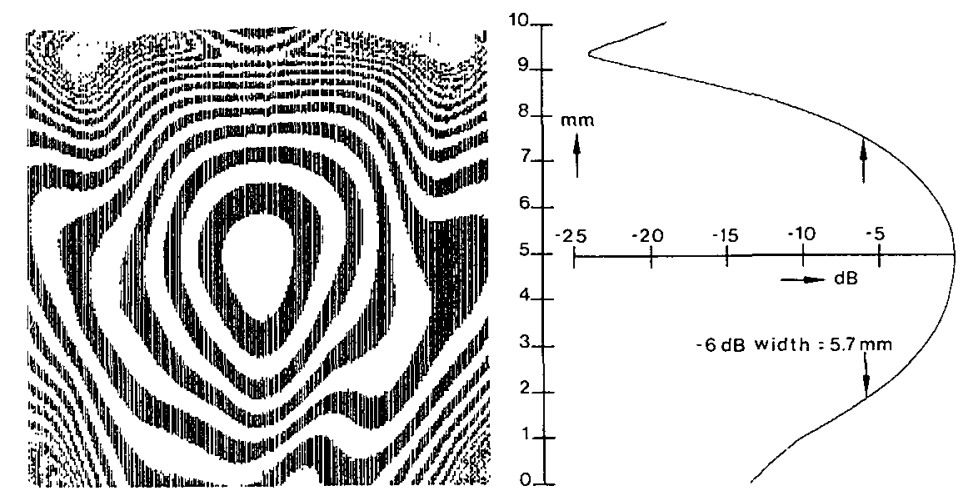

Fig. 2.3 : Two-dimensional pressure distribution of a $3.8 \mathrm{MHz}$ CW-transducer at a distance of $30 \mathrm{~mm}$. Each black-towhite transition indicates a change in signal level of $1.5 \mathrm{~dB}$. Along the beam-plot the signal level over a cross-section through the center of the ultrasound beam is depicted in analog form. At the distance of $30 \mathrm{~mm}$ the $-6 d B$ width of the beam is equal to $5.7 \mathrm{~mm}$.

only half a disc the distribution is not axi-symmetric. The transitions from white to black and from black to white indicate a change in amplitude level of $1.5 \mathrm{~dB}$ with respect to the 
peak amplitude near the axis of the sound beam. This picture is made by scanning automatically the sound field at a distance of $30 \mathrm{~mm}$ from the transducer with a small wide band microphone with an active surface of $1 \mathrm{~mm}^{2}$. Actually the transducer is moved in an angular way in both directions to maintain the same distance between transducer and microphone. The output signal of the microphone in analog form is depicted alongside the twodimensional intensity scan reflecting the intensity in one cross-section. It will be clear from this picture that it is difficult to define the width of the beam. Taking as reference the $-6 \mathrm{~dB}$ level with respect to the peak level, we can conclude (Eig. 2.3) to a beamwiath of $5.7 \mathrm{~mm}$.

\subsection{DOPPLER PRINCIPLE}

If sound waves with frequency $f_{e}$ are reflected by a moving acoustical interface then the moving interface will induce a shift $f_{D}$ in the sound frequency which is proportional to the velocity of the interface. To demonstrate this relation consider a single wave impinging perpendicular on an interface moving with velocity $v$. The wave will have a velocity relative to the transducer of $\mathrm{ctv}$ while after reflection the speed will be changed to $\mathrm{c}-\mathrm{v}$. The time necessary for total reflection of the wave is equal to $\lambda /(\mathrm{c}+\mathrm{v})$. In the meantime the front of the wave has covered the distance $\lambda$ ' where

$$
\lambda^{\prime}\left(f_{e}+f_{D}\right)=c
$$

Therefore

$$
\begin{aligned}
& \lambda /(c+v)=\lambda^{\prime} /(c-v) \\
& E_{D}=2 f e^{v /(c-v)}
\end{aligned}
$$

where use is made of the relations $\lambda=c / f_{e}$ and $\lambda^{\prime}=c /\left(f_{e}+f_{D}\right)$. since $c$ is in the order of $1500 \mathrm{~m} / \mathrm{sec}$ while blood velocities will generally not exceed $1 \mathrm{~m} / \mathrm{s} \quad(\mathrm{v}<\mathrm{c})$ the expression may be 
16

reduced to:

$$
f_{D}=2 f e^{v / c}
$$

If the interface and the sound waves are not moving along the same line we should take in consideration the angle between both velocity vectors. Let this angle be $\alpha$ then the Doppler expression will be

$$
f_{D}=2 f_{e^{v}} \cos \alpha / c
$$

From this expression it can be concluded that the change in frequency will be negative if the interface is moving in the same direction as the impinging sound and positive if the interface moves in the opposite direction. This means that the frequency of the sound backscattered by small moving blood particles is either higher or lower than the frequency of the emitted sound. Assuming a blood velocity $v^{\star} \cos \alpha$ of $50 \mathrm{~cm} / \mathrm{s}$, then the Dopplershift based on an emission frequency of $6 \mathrm{MHz}$ will be $4 \mathrm{kHz}$. Some signal processing is required to make this Doppler shift audible.

The Doppler principle is employed to assess transcutaneously velocities in major arteries. Ultrasound is emitted in a narrow beam aimed at the artery. The backscattered and in frequency shifted sound waves are sensed by the same or a second transducer. Since not all blood particles along the cross-section of an artery are moving with the same speed with respect to the transducer we will receive a spectrum of Doppler frequencies. Because the blood velocity in an artery changes during a cardiac cycle the spectral distribution of Doppler frequencies will be a function of time. The rate of change depends on the artery under investigation but, in general, the frequency content of the velocity waveform is 1 imited to $20 \mathrm{~Hz}$. (McDonald, 1974; Farrar, 1979; Skidmore, 1980). 


\subsection{DOPPLER SYSTEMS}

The Doppler principle as introduced in the previous section is applied in different types of Doppler systems. Presently the continuous wave system ( $\mathrm{CW}$-system), emitting ultrasound with constant amplitude and frequency, is most widely used. However, it is not necessary to emit ultrasound continuously to retain all the information. Sampling the Doppler signal (Blackman, 1959; Jerri, 1977) with a frequency of at least twice the anticipated maximum Doppler frequency is sufficient to restore the original signal completely (Nyquist Theorema). This principle is applied in pulsed Doppler systems where a short burst of ultrasound is emitted repeatedly. The pulse repetition frequency (PRF) is then the sampling frequency for the Doppler signal. Each emission results in a single sample point of the Doppler signal. Emitting a short burst offers the possibility of range discrimination, like in pulsed echo systems, by taking only into account the returned signals with a given delay in relation to the time of emission. Based on the known speed of sound in tissues the elapsed time delay can be converted into a distance. 



\section{CW-DOPPLER SYSTEMS}

\subsection{INTRODUCTION}

Although $C w$-Doppler systems are no part of this study we would like to discuss its basic technical principles because of its simplicity as compared to (multi-gate) pulsed Doppler systems. It will therefore be easier to demonstrate the different steps in the involved signal processing and to show their effect on the system output depending on the recording conditions, such as signal-to-noise ratio (SNR), beamwidth, sample volume and bandwidth of the Doppler signal.

\subsection{RECEIVER, DEMODULATOR}

In fig. 3.1 the basic block diagram of a CW-system is depicted. A local oscillator provides the emitter stage with a stable signal both in carrier frequency and in amplitude. The emitter drives the emitting crystal with its natural oscillating frequency. The acoustic signal reflected and backscattered

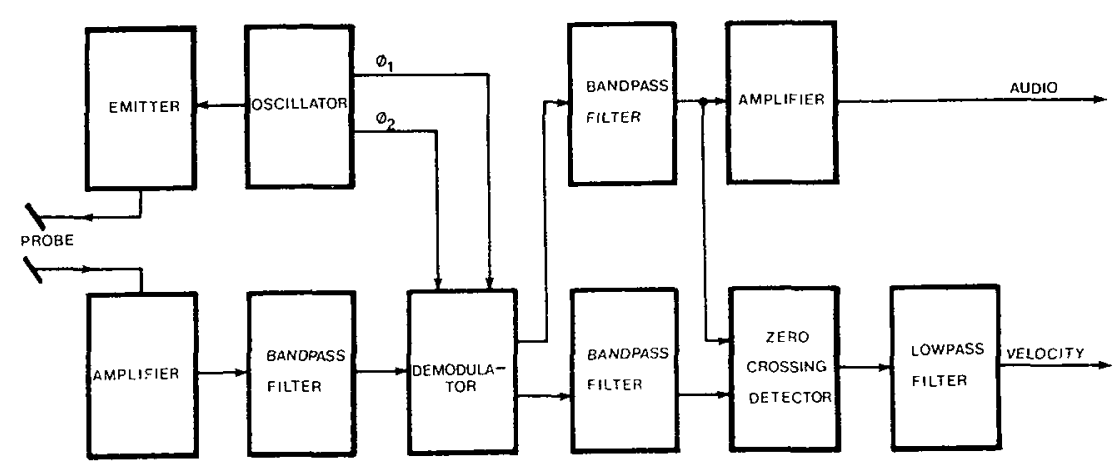

Fig. 3.1 : Block-diagram of a CW-Doppler system. To retain directionality the received signal is demodulated by two in quadrature reference signals. To detect the average Doppler frequency representing the average velocity with respect to the transducer a zero crossing detector is utilized. 
by acoustic interfaces is picked up by a second crystal and amplified by the receiver circuitry. The relevant frequencies in the returned signal will be within a narrow band around the emitting frequency (see expr. 2.5). Frequencies outside this range can be attributed to noise and should be suppressed to improve the signal-to-noise ratio. This suppression is partly executed by the employed electro-acoustic transducers, owing to its narrow band characteristics, and partly by the receiver stage (Atkinson, 1976). Considering only thermal noise then the equivalent noise voltage $\bar{v}_{n}$ at the input of the receiver can be expressed as:

$$
\overline{\mathrm{v}}_{\mathrm{n}}^{2}=4 \mathrm{KTRB}
$$

where $\quad K$ is the constant of Boltzmann $\left(1.38 * 10^{-23} \mathrm{~J} / \mathrm{K}\right.$ )

$T$ is the temperature (K)

$R$ is the equivalent noise resistance of the receiver stage $\left(J / A^{2} S\right)$

$B$ is the bandwidth $\left(\mathrm{s}^{-1}\right)$

Assuming that the input resistance of the receiver stage is properly matched to the impedance of the transducer the noise voltage can only be reduced by reducing the bandwidth $B$ without deteriorating the signal. An effective noise voltage in the order of $0.1 \mu \mathrm{V}$ is then attainable for a bandwidth of $20 \mathrm{kHz}$ and a source resistance of $25 \Omega$.

In order to retreive the Doppler information the bandfiltered and amplified received signal is demodulated using two in quadrature reference signals derived from the oscillator:

$$
s_{d}(t)=s_{r}(t) \exp \left(j 2 \eta f_{e} t\right)
$$

where $\mathrm{f}_{\mathrm{e}}=$ emission frequency

$s_{r}(t)=$ high frequency received signal

$s_{d}(t)=$ low Erequency demodulated signal.

The modulation with two in quadrature reference signals, i.e. two signals with the same amplitude and frequency but shifted 
$90^{\circ}$ in phase with respect to each other, enables the conservation of the directional information. This results in two demodulated signals while the phase relation indicates the direction of the detected flow with respect to the transducer (McLeod, 1967). To achieve a further reduction in the contribution of (thermal) noise and to suppress higher harmonics due to the demodulation process the demodulator is followed by a low-pass Eilter with a cut-off frequency exceeding the maximum anticipated Doppler frequency (in the order of $10 \mathrm{kHz}$ ).

The received signal does not only contain signals originating from blood particles (mainly the erythrocytes) but also signals reflected by stationary and slowly moving interfaces like tissue boundaries and vessel walls. The power of these reflected signals tends to obscure the low power Doppier signals backscattered by the blood particles. However, the Doppler frequency range of the reflected signals is generally limited to low frequencies and can therefore be rejected by a high-pass filter. The combination of low-pass filter for noise rejection and high-pass filter to suppress vessel wall induced Doppler signals results in a band-pass filter. The band-pass filter for both in quadrature demodulated Doppler signals should be identical to maintain the amplitude and phase relationship between both signals. The selection of the lower cut-off frequency of the band-pass filter depends on the employed emission frequency in combination with the structures under investigation. Since the cut-off frequency will limit the capability of the system to detect low velocities with respect to the transducer it has to be as low as possible. Unfortunately no general rule can be developed for the selection of the lower cut-off frequency of the band-pass filter. For a $5 \mathrm{MHz}$ Dopoler system for peripheral subcutaneous investigation the $-3 \mathrm{~dB}$ cut-off frequency will be in the order of $300 \mathrm{~Hz}$. This implies that the lowest jetectable velocity with respect to the probe is in the order of $3.5 \mathrm{~cm} / \mathrm{s}$. 


\subsection{AUDIO-EVALUATION}

The demodulated high-pass filtered signals contain Doppler frequencies within the audio frequency range. Aural evaluation of the Doppler signal is possible by connecting one of the in quadrature Doppler signals to an audio amplifier. Proper aural evaluation may be impaired if the detected signal contains Doppler information from both a vein and an artery. However, generally the vein and artery will have an opposite flow direc-

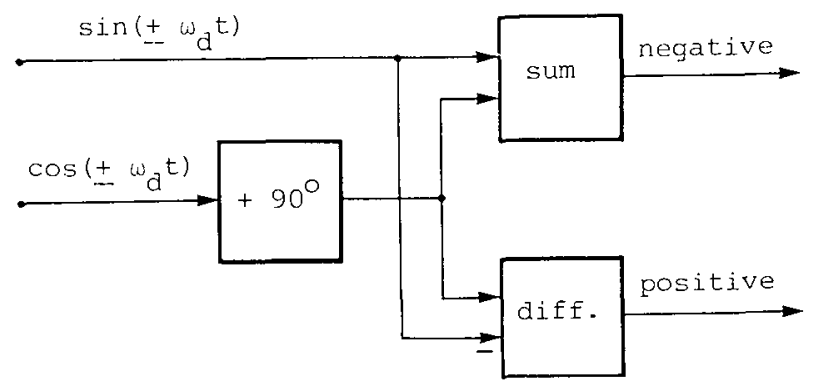

Fig. 3.2 : With a $90^{\circ}$ phase-shifter separate audio-outputs for positive and negative Doppler frequencies can be obtained from the in quadrature Doppler signal.

tion with respect to the ultrasonic transducer. Under these circumstances both signals can be separated by a proper combination of both in quadrature signals (fig. 3.2). For a single angular Doppler frequency " $D=2 \pi f_{D}$ an in quadrature Doppler signal with amplitude $A_{0}$ can be expressed as:

$$
\begin{aligned}
s_{d}(t) & =A_{0} \exp \left(j \omega_{D} t\right) \\
& =A_{O} \cos \left(\omega_{D} t\right)+A_{O} \sin \left(\omega_{D} t\right)
\end{aligned}
$$

Phase shifting the real part of $s_{d}(t)$ over $90^{\circ}$ is equal to:

$$
A_{O} \cos \left(\omega_{D} t+\pi / 2\right)=A_{O} \operatorname{sgn}\left(\omega_{D}\right) * \sin \left(\omega_{D} t\right)
$$

where

$$
\begin{array}{rllll}
\operatorname{sgn}\left(\omega_{D}\right) & =1 & \text { if } & \omega_{D}>0 \\
& =0 & \text { if } & \omega_{D}=0 \\
& =-1 & \text { if } & \omega_{D}<0
\end{array}
$$


The sum of the real part of the Doppler signal shifted over $90^{\circ}$ and the imaginaire signal will be:

$$
\begin{array}{rlrl}
A_{O} \operatorname{sgn}\left(\omega_{D}\right) * \sin \left(\omega_{D} t\right)+A_{O} \sin \left(\omega_{D} t\right) & =0 & \text { if } \omega_{D}<0 \\
& =2 A_{O} \sin \left(\omega_{D} t\right) \text { if } \omega_{D}>0
\end{array}
$$

while the difference of both signals results in:

$$
\begin{aligned}
A_{0} \operatorname{sgn}\left(\omega_{D}\right) * \sin \left(\omega_{D} t\right)-A_{O} \sin \left(\omega_{D} t\right) & =-2 A_{O} \sin \left(\omega_{D} t\right) & & \text { if } \omega_{D}<0 \\
& =0 & & \text { if } \omega_{D}>0
\end{aligned}
$$

Depending on the sign of the Dopplex frequency, i.e. on the direction of the velocity with respect to the transducer, the Doppler signal will appear at one of the outputs, thus enabling aural evaluation of velocities either advancing or receding with respect to the ultrasonic probe. The interpretation of the audio signals may cause some problems if advancing and receding velocities are observed simultaneously or if the direction of the blood flow is a function of the caraiac cycle.

\subsection{FREQUENCY ANALYSIS OF THE DOPPLER SIGNAL}

Although the ear is very sensitive for the spectral content of the Doppler signal, it is incapable of giving an objective evaluation of the signals because of its property to perceive differences in (frequency-dependent) amplitudes non-linearly. Therefore, a real-time spectral analyzer is generally connected to the output of the network as Jescribed in the preceding section (Baskett, 1977; Reneman, 1979a; Reneman, 1979b; Rittgers, 1980). To record the spectral content as function of time the output of the spectral analyzer is represented in shades of grey where the highest intensity represents the highest amplitude for a given frequency (fig. 3.3). In the past years several spectral analyzers have been developed especially dedicated to the analysis of Doppler signals. Some of them incor- 
24

porate the ability to separate forward and reverse velocities (frequency splitter) as presented in the previous section. The restraints on the performance of the spectral analyser depends on the characteristics of the employed Doppler system and the

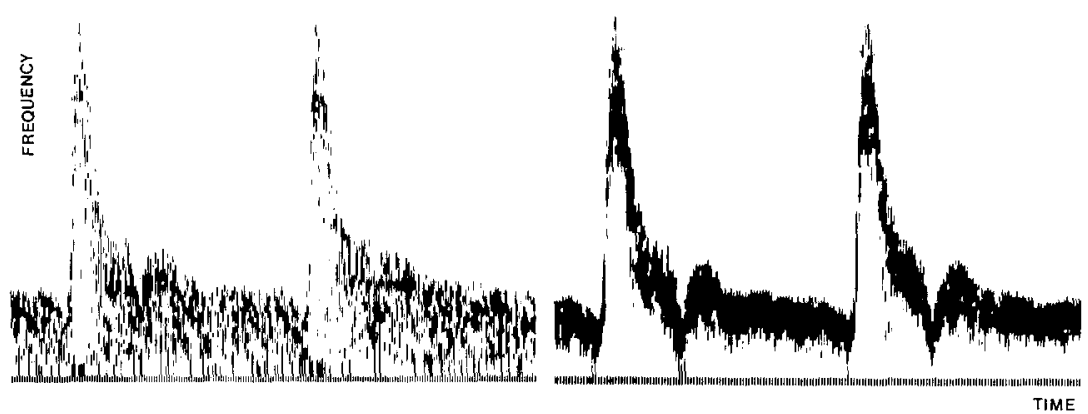

Fig. 3.3 : Sonogram (frequency versus time) as obtained with a CW-system (top) and pulsed Doppler system (bottom) from the common carotid artery. The intensity of the signal tends to concentrate towards the maximum frequency of the signal.

hemodynamic behaviour of the investigated vessels. To appreciate this properiy one should consider the resolution in time and frequency and the required frequency range. Generally, the time required to process the data is related to the number of analysis points (Bin's) in the frequency domain. Application of smart processing algorithms (East Fourier Transform analyser, time compression analyser) will bring down the processing time. However, data-processing can only be initiated after data acquisition has been completed. Employing a double input memory allows for simultaneous acquisition and processing. The time elapsing between two consecutive output sweeps (temporal resolution) will be the longest of acquisition and processing time. Assuming that processing time is not the limiting factor (an appropriate number of Bin's) then the data-acquisition time dictates both temporal and spectral (width of a Bin) resolution, while the maximum analysis range equals Bin-width times number of Bin's. Under this condition a 128 points FFr-analyser with a Bin-width of $60 \mathrm{~Hz}$ will have an analysis range in the order of $7.5 \mathrm{kHz}$ while 60 spectra per second are produced. 
The number of spectra per second should be in accordance with the anticipated rate of change of the observed velocity signal. Generally, in cardiac applications the rate of change will be higher than in peripheral blood flow measurements. This leads to the conclusion that the width of a Bin in cardiac flow analysis will be larger since resolution in the frequency domain should be sacrificed in favor of temporal resolution. Depending on the assessed velocities the maximum anticipated frequency range of the Doppler signals will be lower because of the lower employed emission frequency to compensate for the frequency dependent attenuation of ultrasound by tissues. Therefore, the relative resolution (width of a Bin with respect to the maximum frequency range) will be poorer in cardiac flow analysis as compared to peripheral flow analysis. Even in peripheral flow assessment the highest possible emission frequency should be preferred to allow for the best relative resolution. If the achieved temporal resolution is far better than the minimum requirement, then they may be brought in accordance with each other by averaging consecutive spectra to reduce spectral variance. A good relative spectral resolution combined to a low spectral variance is especially of importance if the analyzer output is used to qualify the assessed velocity distribution based on the spectral width of the Doppler signal (Forster, 1977; Hutchison, 1981).

\subsection{DETECTION OF AVERAGE VELOCITY}

Frequency analysis of the detected Doppler signal reveals the spectral content of the signal as function of time. This requires, however, a complicated and expensive system. If one is only interested in a specific feature of the Doppler signals, e.g. the average frequency representing the average velocity, relative simple dedicated circuitry may be employed. Although the procedure will discard information about the frequency distribution detection of average Doppler frequency is widely used because it requires simple recording facilities and 
the output, assumed to be proportional to the average velocity in the investigated vessel, reflects a familiar physiological parameter.

In order to calculate the average Doppler frequency $\overline{\mathrm{F}}_{\mathrm{D}}$ one has to evaluate the expression:

$$
\bar{f}_{D}=\frac{-\infty}{\int_{\infty}^{\infty} s_{d}(f) d f}
$$

where $S_{d}(f)$ stands for the spectral distribution of the Doppler signal $s_{d}(t)$. Under pulsatile flow conditions $s_{d}(f)$ and therefore $\bar{f}_{D}$ will be a function of time. Assuming that the frequency content of the velocity signal averaged over the cross-sectional area is limited to $20 \mathrm{~Hz}$ then it suffices to calculate $\mathrm{S}_{\mathrm{d}}(\mathrm{f})$ over an interval of $25 \mathrm{~ms}$. Shortening of this observation interval will result in an increase of the variance in $\bar{F}_{D}$ while a too long observation interval will discard the higher frequencies present in the velocity signal.

Usually, the calculation of $\overline{\mathrm{f}}_{\mathrm{D}}$ is executed in the time domain rather than in the frequency-domain (Arts, 1972; Roevros, 1974; Angelsen, 1975). Since it involves the division by the time-dependent Doppler power the output of the frequency detector circuit might become instable for low power of the Doppler signals. To circumvent this problem $\overline{\mathrm{F}}_{\mathrm{D}}$ is set to zero if the Doppler power drops below a certain threshold.

Evaluation of expression 3.8 results in a correct estimate for the average Doppler frequency in the absence of noise independent of the spectral distribution $S_{d}(f)$. However, a bias will show up if the Doppler signal is contaminated by noise (Gill, 1979 ; Gerzberg,1980). The estimated average signal frequency $\overline{\mathrm{f}}_{\mathrm{s}}$ will be equal to:

$$
\hat{\bar{f}}_{s}=\left(\hat{M}_{1 s}+\hat{M}_{1 n}\right) /\left(\hat{M}_{0 s}+\hat{M}_{0 n}\right)
$$


where $\begin{aligned} M_{1 s} & =\int_{-a}^{\infty} f s_{d}(f) d f \\ M_{1 n} & =\int_{-\infty}^{\infty} f s_{n}(f) d f \\ M_{0 s} & =\int_{-\infty}^{\infty} s_{d}(f) \text { af }=s \\ M_{0 n} & =\int_{-\infty}^{\infty} s_{n}(f) d f=N\end{aligned}$

In this expression $S_{n}(f)$ stands for the spectral distribution of the noise and $S$ and $N$ for the signal power and noise power, respectively. The symbol $\wedge$ indicates that estimated values based on a given observation interval rather than the true quantities are usea. Assuming a uniform spectral distribution of the noise over the frequency range $|E|\left\langle E_{M}\right.$, where $F_{M}$ stands for the upper cut-off frequency of the Doppler bankpass filter, the expected value for $\hat{A}_{1 n}$ will be zero and expression 3.9 transforms into (Gill, 1979; Gerzberg, 1980):

$$
\hat{\mathrm{E}}_{s}=M_{1 s} /(S+N)
$$

Therefore, the expected value of $\overline{\mathrm{f}}_{\mathrm{S}}$ will deviate from $\overline{\mathrm{f}}_{\mathrm{D}}$. The estimator error depends on the signal-to-noise ratio (SNR). For a $S N R=20$ the average Doppler frequency will be underestimated by approximately $5 \%$. The error in the estimator output will vanish for increasing signal-to-noise ratio.

The above viaw applies for the situation that a directional Erequency estimator is used. If the Doppler signals are separated in Eorward and reverse Doppler signals (section 3.3), then for both Doppler signals non-directional frequency estimaters can be used i.e. the integrals in expression 3.4 are only evaluated for positive frequencies. The expected value for $\hat{M}_{\text {In }}$ will transfer into $N . f_{M} / 2$ and the estimator output will be equal to (Gill, 1979; Gerzberg, 1980):

$$
\hat{\mathrm{f}}_{\mathrm{S}}=\left(\hat{\mathrm{M}}_{1 \mathrm{~S}}+\mathrm{N} \mathrm{f}_{\mathrm{M}} / 2\right) /(\mathrm{S}+\mathrm{N})
$$


The error in the estimator output will not only depend on the signal-to-noise ratio but also on the value of $\bar{f}_{D}$ with respect to $\mathrm{f}_{M} / 2$. For average Doppler frequencies less than $f_{M} / 2$ the average Doppler frequency will be overestimated while for average Doppler frequencies exceeding $\mathrm{f}_{\mathrm{M}} / 2$ the average Doppler frequency will be underestimated. Table 3.1 summarizes the effect

\begin{tabular}{|l|rrrrrrrrr|}
\cline { 2 - 9 } \multicolumn{1}{c|}{} & \multicolumn{10}{c|}{$\bar{f}_{D_{M} / f_{M}}$} \\
\hline SNR(dB) & 0.1 & 0.2 & 0.3 & 0.4 & 0.5 & 0.6 & 0.7 & 0.8 & 0.9 \\
\hline 10 & 36.4 & 13.6 & 6.1 & 2.3 & 0.0 & -1.5 & -2.6 & -3.4 & -4.0 \\
20 & 4.0 & 1.5 & 0.7 & 0.2 & 0.0 & -0.2 & -0.4 & -0.4 & -0.4 \\
30 & 0.4 & 0.1 & 0.1 & 0.0 & 0.0 & -0.0 & -0.0 & -0.0 & -0.0 \\
\hline
\end{tabular}

Table 3.1: Relative error (expressed in percents) in the output of a non-directional frequency detector based on the spectral distribution as function of the signal-tonoise ratio (SNR) and the average Doppler frequency $\bar{f}_{D}$ with respect to the maximum frequency $f_{M}$.

of the signal-to-noise ratio on the estimator output as function of $\bar{f}_{D} / f_{M}$. It can be concluded that for low average Doppler frequencies (as will occur in the diastolic phase of the cardiac cycle) the estimator error due to noise is substantial (Gerzberg, 1980). If a priori knowledge about the noise characteristics ( $M_{I n}$ and $M_{0 n}$ ) are available then the error introduced by noise can be corrected for (Gerzberg, 1980) by reducing the numerator and denominator in expression 3.9 by $\hat{M}_{1 n}$ and $\hat{M}_{0 n}$ ' respectively. $\hat{M}_{I n}$ and $\hat{M}_{O n}$ can easily be obtained if the probe is disconnected (assuming that the probe does not contribute significantly to the noise). This procedure will not reduce the variance in the estimator output.

The method as described above to derive an estimate for the average Doppler frequency, based on expression 3.9, is employed in a few systems only because of the technical problems encountered. Most systems employ an estimator based on the density of zero crossings (Lunt, 1975). For a single Doppler frequency the output of this estimator will be unbiased i.e. the time average of the estimated value equals the expected value. However, it 
can be shown that for a non-directional frequency estimator based on the zero crossing density the output equals (Rice, 1944):

$$
\hat{\bar{f}}_{s}=\left(\int_{-\infty}^{\infty} f^{2} s_{d}(f) d f / \int_{-\infty}^{\infty} S_{d}(f) d f\right)^{\frac{1}{2}}
$$

The bias in the estimator output, henceforth, depends on the shape of the Doppler frequency spectrum (Peronneau,1974a). The estimator error is small for a frequency spectrum which is narrow with respect to the average frequency but will be substantional (exceeding 10\%) for a Doppler spectrum uniform up to a given maximal frequency i.e. a wide spectrum. A frequency estimator based on the density of zero crossings can be made directional by taking into account the in quadrature filtered Doppler signals (Mcleod, 1967) or by evaluating separately the forward and reverse flow outputs of the frequency splitter (section 3.3). The effect of signal bandwidth on the estimator output suggests the application of the second method especially in the presence of noise. However, no general analytic solution is yet obtained to demonstrate the effect of (wide-band) noise on the expected value and variance of estimator output in both approaches (Angelsen, 1980b). Assuming a rectangular spectral distribution of the Doppler signal around a frequency af ${ }_{M}(a<1)$ with width $2 b f_{M}(b<a)$, where $f_{M}$ is again the upper cut-off frequency of the Doppler filter and assuming a uniform spectral distribution for the noise up to $f_{M}$, then the expected estimator output of the non-directional zero crossing detector, based on expression 3.12 , will be equal to:

$$
\frac{\hat{\mathrm{f}}}{S}_{S}=\mathrm{f}_{M}\left(\left(\left(\mathrm{a}^{2}+\mathrm{b}^{2} / 3\right) \mathrm{S}+\mathrm{N} / 3\right) /(\mathrm{S}+\mathrm{N})\right)^{\frac{1}{2}}
$$

For $N=0$ (no noise) the expected value for the average signal frequency will be equal to:

$$
\begin{aligned}
\hat{\mathrm{f}}_{S} & =a \mathrm{f}_{M}\left(1+b^{2} / 3 a^{2}\right)^{\frac{2}{2}} \\
& =\overline{\mathrm{f}}_{\mathrm{D}}\left(1+\mathrm{b}^{2} / 3 \mathrm{a}^{2}\right)^{\frac{1}{2}}
\end{aligned}
$$


confirming that the error in the estimator output is proportional to the relative width (b/a) of the signal spectrum. In the presence of noise the expected estimator output is equal to:

$$
\hat{\bar{f}}_{S}=\overline{\mathrm{f}}_{D}\left|\left(\left(1+\mathrm{b}^{2} / 3 \mathrm{a}^{2}\right) \mathrm{s}+\mathrm{N} / 3 \mathrm{a}^{2}\right) /(\mathrm{S}+\mathrm{N})\right|^{\frac{1}{2}}
$$

Defining the relative error $\mathrm{E}_{\mathrm{R}}$ in the estimator output as:

$$
E_{R}=100\left(\hat{\bar{f}}_{S}-\bar{f}_{D}\right) / \bar{E}_{D}
$$

then we may compute $\mathrm{E}_{\mathrm{R}}$ for a fixed relative signal bandwidth $(b / a=0.2)$ as function of $a$ and $s / N$ (table 3.2). It can be concluded that for low signal-to-noise ratios and for low values of a the relative error $\mathrm{E}_{\mathrm{R}}$ in the estimator output is substan-

\begin{tabular}{|r|rrrrrrrrr|}
\hline & \multicolumn{10}{|c|}{$\vec{f}_{D} / f_{M}$} \\
\hline SNR $(a B)$ & 0.1 & 0.2 & 0.3 & 0.4 & 0.5 & 0.6 & 0.7 & 0.8 & 0.9 \\
\hline 10 & 98.9 & 29.5 & 12.1 & 5.4 & 2.1 & 0.3 & -0.8 & -1.6 & -2.1 \\
20 & 15.5 & 4.2 & 2.0 & 1.2 & 0.8 & 0.6 & 0.5 & 0.4 & 0.4 \\
30 & 2.3 & 1.0 & 0.8 & 0.7 & 0.7 & 0.6 & 0.5 & 0.6 & 0.6 \\
\hline
\end{tabular}

Table 3.2: Relative error (expressed in percents) in the output of a non-directional zero crossing detector as function of the signal-to-noise ratio (SNR) and the average Doppler frequency $\vec{f}_{D}$ with respect to the maximum frequency $f_{M}$.

tial (more than 10\%). To avoid detection of zero crossings (and an erroneous output) under poor signal-to-noise ratio conditions, the signal is compared to a threshold unequal to zero. This threshold is adjusted such that in the absence of true Doppler signals no zero crossings are detected. The noise will regain its influence on the detector output if the Doppler signal plus noise are able to activate the zero crossing detector.

If non-directional. frequency estimators are employed in combination with a frequency splitting network (section 3.3), the outputs will represent the average negative and positive signal 
frequency respectively. If the difference between both outputs is taken in order to obtain a directional velocity waveform an additional error is introduced. This error will appear if simultaneously positive and negative average signal frequencies zero are detected. The error in the composite detector output due to subtraction can only be prevented if the signal power at the input of both detector circuits is taken into account properly.

\subsection{DISCUSSION}

Doppler systems based on the continuous wave mode of operation are widely used due to their relative simplicity and the ease with which an output signal can be obtained which is, at least, indicative for the velocity as function of time averaged over the cross-sectional area of the investigated blood vessel. However, this simplicity evokes several sources of error which make it difficult to evaluate properly the assessed signals. The signals are returned by all the tissues and vessels insonated by the ultrasonic beam. The ultrasonic beam however is not homogeneous (wells, 1969) and the ultrasound intensity will vanish for increasing depths due to absorption and reflection. Therefore, blood particles in shallow vessels will return more acoustic signal then blood particles in deeper vessels. Because of the inhomogenity of the ultrasound beam not all blood particles will receive the same acoustic signal. However, for the detection of the average velocity it is assumed that all blood particles return an acoustic signal with the same intensity towards the probe (Roevros, 1974). The situation becomes even more complicated if two vessels are located within the ultrasonic beam. with dedicated equipment (spectral analyzer, frequency splitter) the inherent complication can be circumvented if both vessels maintain an opposite flow cirection with respect to the ultrasonic transaucer throughout the cardiac cycle. The commonly employed zero crossing detector for the average frequency and, henceforth, the average velocity with re- 
32

spect to the probe is biased where the bias depends on the actual shape of the Doppler frequency spectrum. Moreover, the average frequency detectors are susceptible for noise. However, the noise level with respect to the signal level can be kept small by properly designing the receiver and demodulator stage. The upper cut-off frequency of the bandpass filter for the Doppler signals should be selected as low as possible to obtain a reliable estimator output. Some of the drawbacks mentioned above apply for $\mathrm{C}$-systems only (absence of depth information), other drawbacks appear in both $\mathrm{CW}$ and pulsed Doppler systems (inhomogenity of ultrasound beam). A major advantage of a pulsed Doppler system is its ability to provide information about the location and dimensions of the investigated blood vessels. However, a pulsed Doppler system requires a more complicated design approach. 


\section{PULSED DOPPLER SYSTEMS}

\subsection{BASIC CONCEPTS}

The basic configuration of a CW-system (oscillator, emitter, transducer, receiver, demodulator, high-pass filter, average Erequency detector) is simular to that of a pulsed Doppler system (fig. 4.1). However, the way the different sections are operated are quite different. Before we will discuss this we

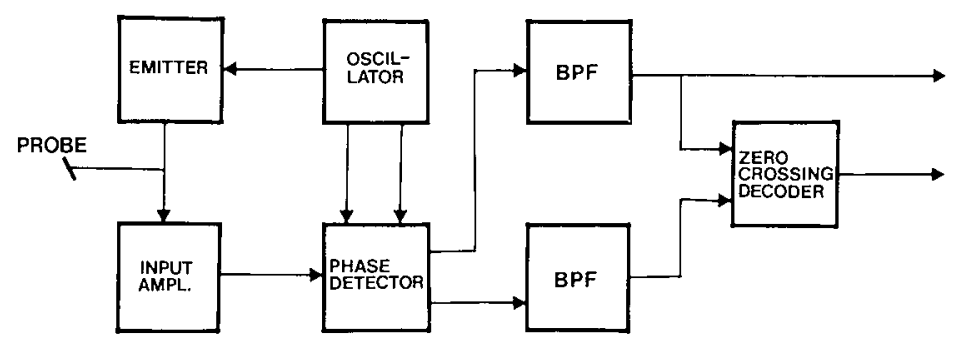

Fig. 4.1 : Block-diagram of a pulsed Doppler system. The phase-detector contains demodulator, , low-pass filter and sample and hold circuit.

would like to summarize some basic concepts in signal analysis. The Doppler signal as it appears at the output of the demodulator circuit of a continuous wave system is completely described by $2 f_{M}$ equidistant sample-points per second where $f_{M}$ is the maximum anticipated frequency in the detected Doppler signal (Nyquist-theorema). The original wave-form can then be restored by a low-pass filter with a cut-off frequency of $f_{M}$. Sampling of the demodulated signal can be performed with a sample-andhold amplifier with a short data-acquisition time. The value of the sampled signal is then retained in the sample-and-hold circuit until it is updated. It reflects the (co)sine of the instantaneous phase-difference between the received and reference signal, weighted by the amplitude of the received signal. To detect the phase-difference properly the demodulator output is averaged over a couple of periods of the reference signal employing a low-pass filter. 
If the effective duration of the impulse response of the low-pass filter (Gabor, 1946) is in the order of 1 us then the phase-difference between both signals will not vary significantly within this averaging interval. What happens with the received signal outside the averaging interval will not affect the sample value. It is therefore unnecessary that the signal received from particles within a blood vessel remains continuously available, but it should be present when the sample is taken. This condition is met when ultrasound is emitted in bursts rather than continuously. Then the duration of emission determines the time-interval that the signal returned by a given acoustic interface is available. Pulsed ultrasound systems, however, have the capability to resolve reflectors at different depths because of the required time of flight. By taking in consideration the propagation speed of ultrasound in tissues, the time elapsing between emission and sampling the phase difference sets the depth of investigation. The pulse repetition frequency (PRF) acts as sampling frequency for the Doppler signal and should be at least twice as high as the anticipated Doppler frequency shift. On the other hand the reflected and backscattered signals, received from the region of interest, should be received before the next burst can be emitted to

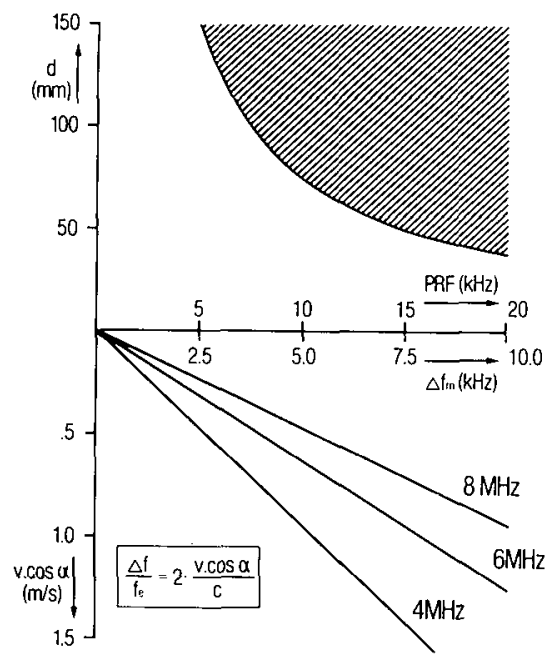

Fig. 4.2 : Ambiguity-diagram (depth vs PRF vs velocity). The depth of investigation limits the pulse repetition frequency, while the $P R F$ sets an upper bound to the velocity with respect to the probe. 
avoid range ambiguity. The selected depth of investigation sets therefore an upper bound to the PRF. The relation between maximum velocity and maximum depth at which this velocity can be detected unambiguously is depicted in fig. 4.2. From this figure it can be concluded that for a depth of $75 \mathrm{~mm}$ the maximum PRF (based on a propagation speed of sound in tissue of 1500 $\mathrm{m} / \mathrm{s}$ ) will be $10 \mathrm{kHz}$ and the maximum Doppler frequency range 5 $\mathrm{kHz}$. For a system with an emission frequency of $6 \mathrm{MHz}$ the maximum velocity with respect to the transducer $(v \cdot \cos \alpha)$ is limited to $.625 \mathrm{~m} / \mathrm{s}$. Extension of the velocity range of a pulsed Doppler system is possible by selecting a lower emission frequency (fig. 4.2). However, under specific restrictions the velocity range of pulsed Doppler systems can be enlarged by employing dedicated processing of the Doppler frequency information (see chapter 6).

\subsection{TRANSDUCER}

Since in a pulsed Doppler system short bursts of ultrasound are emitted and the time elapsing between two consecutive emissions is used to pick up the returned signal the same transducer can be alternately used as emitter and receiver. The transducer should be able to accommodate the short pulse i.e. its bandwidth should be reciprocal to the duration of emission. Transducers made of piezo-ceramic material have a too small bandwidth for a burst with a duration in the order of 1 us. The bandwidth can be enlarged by properly backing the transducer (Kossoff, 1966) and/or applying properly dimensioned frontlayers (Angelsen, 1975). A draw-back of enlarging the transducerbandwidth by backing is the decreasing efficiency of the transducer i.e. the capability to convert electric in acoustic energy and vice-versa (Angelsen, 1975). The received electric signal power will be reduced by a factor of 4 if the efficiency is reduced by a factor of 2 as compared to a $\mathrm{CW}$-transducer activated by the same power of the emission signal. The reduction in efficiency can be compensated for by increasing the amplitude of the emission signal. For a given amplitude of the electric 
signal applied to the transducer, pulsed operation will reduce the emitted power averaged over time. However, the level of the detected Doppler signal (under the same amplification conditions) will not diminish proportional with respect to the reduction in emitted power.

The duration of emission sets the axial resolution of the system provided that the transducer, input amplifier and demodulator are able to accommodate this short pulse iJorgesen, 1973). Based on a speed of sound in tissue of $1500 \mathrm{~m} / \mathrm{s}$ an emission duration of $4 / 3 \mu \mathrm{s}$ will result in an axial resolution of 1 $\mathrm{mm}$. The lateral resolution depends on the local width of the ultrasonic beam. Both resolutions should be in the same order to obtain the best results. The effect of both dimensions on the detected signals will be discussed in section 4.5 .

\subsection{RECEIVER}

The weak electrical signals as produced by the transducer are amplified in the receiver stage. The gain is made timedependent i.e. the amplification increases with time to correct for the attenuation of ultrasound, increasing with time of flight due to absorption by the tissues, which is in the order of $1 \mathrm{~dB} / \mathrm{cm} / \mathrm{MHz}$ (Wells, 1969).

The amplifier should have a bandwidth corresponding to the duration of the emitted pulse. Assuming a bandwidth-time relation of $\Delta f^{*} \Delta t=1$ (Gabor, 1946) the bandwidth should be in the order of $1 \mathrm{MHz}$ for an emission duration of $l \mu s$. This is considerably larger than the receiver bandwidth of a $\mathrm{CW}$-system and henceforth the noise produced by the receiver will increase significantly (see formula 3.1) causing deterioration of the signal-to-noise ratio for the same amplitude of the received signal as compared to a $\mathrm{CW}$-system. This can be only compensated for by increasing the amplitude of the emitted acoustic signal taking in consideration the reduced efficiency of the trans- 
ducer due to acoustic backing for both emission and reception. To compensate for the increase in bandwiath with a factor of 50 (from $20 \mathrm{kHz}$ to $\mathrm{I} \mathrm{MHz}$ ) the received signal amplitude should be 7 times larger. A reduction in transducer efficiency with a factor of 2 requires that the electrical signal for emission should be 2 times larger, taking into account that the reduction in efficiency will affect both emission and reception. Therefore, the amplitude of the electrical signal applied to the transducer should increase 14-fold and the energy of the ultrasound approximately 200 times. To generate a short burst with an amplitude that is 14 times higher as compared to a $\mathrm{CW}-$ system may cause some technical problems. The increase in ultrasound energy with a factor of 100 does not materialize since for a PRF of $20 \mathrm{kHz}$ and an emission duration of 1 us the emitter is only active for $1 / 50$ of the time. Hence the increase in ultrasound energy averaged over time will be only 2-fold due to the reduction in transducer efficiency. For a lower PRF this factor becomes even lower. Shortening the duration of emission does not affect the required ultrasound energy to maintain the same received electrical signal since this will demand again an increase in bandwidth. The above reasoning only applies if the same number of blood cells are investigated. Since the sample volume of pulsed Doppler systems (section 4.5) is considerable smaller than the sample volume of $\mathrm{CW}$-systems pulsed Doppler systems will have a lower signal-to-noise ratio for the same emission frequency.

Pulsed Doppler systems generally employ a narrow ultrasound beam (see section 4.5) implying that for a given ultrasound energy level local energy density may rise well beyond the upper bound set for diagnostic ultrasound equipment. Very narrow beams in terms of wavelengths demands therefore that the total emitted energy is lowered by reducing the PRF and/or by reducing the emitted energy per burst (showtening duration of emission and/or lowering the emission voltage). The last approach will again affect the attainable signal-to-noise ratio. 


\subsection{PHASE DETECTOR}

To detect the instantaneous phase difference between the received and amplified signal and the reference oscillator signal, the received signal is fed into a phase detector. Basically the phase detector consists of a demodulator followed by a low-pass filter to suppress the higher harmonics generated by the demodulator and to detect the phase-difference averaged over a given range interval. This range interval is set by the duration of emission. The effective duration of the impulse response of the low-pass filter (Gabor, 1946) should be therefore equal (Peronneau, 1974a) to the duration of emission. Assuming a bandwidth-time relation $\Delta f \star \Delta t=1$ the bandwidth should be equal to $1 \mathrm{MHz}$ for a pulse duration of $1 \mu \mathrm{s}$ and a cut-off frequency of the filter in the order of $0.5 \mathrm{MHz}$. Increasing the bandwidth and thereby shortening the observation interval will increase the noise contribution (Gill, 1973; Jorgesen, 1973) while extending the observation interval will result in averaging of signals originating from independent locations. Hence, the signal-to-noise level will not improve accordingly while the axial resolution will deteriorate.

The order of the low-pass filter is determined by the required suppression of phase-signals at the emission frequency. To attain a suppression in the order of $60 \mathrm{~dB}$ at $6 \mathrm{MHz}$ a filter of the third order with a cut-off frequency of $0.5 \mathrm{MHz}$ is required. To maintain this suppression the order of the filter should increase if the duration of emission is shortened and the cut-off frequency of the low-pass filter is increased accordingly. To maintain the phase relation between both in quadrature outputs of the phase-detector, both low-pass filters should have the same amplitude and phase characteristics.

At a pre-selected delay with respect to emission corresponding to the selected depth of investigation both phase-signals are stored in an analog memory (sample and hold circuit) to re- 
tain the value until the sample and hold memory is updated. The data-acquisition time of the analog memory should be shorter than the duration of emission to ensure that the output has reached the final value at the time the circuit is switched into the hold-mode. To avoid additional noise the stored data should be maintained at the same value. Both demands (data acquisition time and droop rate of the sample and hold circuit) require the employment of an excellent analog memory.

\subsection{SAMPLE VOLUME}

The demodulator output in combination with the low-pass filter provides the phase-signal as a continuous function of depth. At a pre-selected time-delay with respect to the time of emission this value is stored in the analog memory. The received signals, contributing significantly to the stored phasevalue, originate from a volume, called sampled volume. The size and shape of the sample volume depend on both the axial and lateral resolution of the system. As stated before the axial resolution is dictated by the duration of emission in combination with the filter characteristics of the transducer, the receiver stage and the low-pass filter of the phase detector. The effective duration of the impulse response of this combined filter should be equal to the duration of emission to achieve the best axial resolution with the highest signal-to-noise ratio. Since the cascade of filters with the same frequency characteristics results in a narrower overall characteristic the associated impulse response will be elongated. To obtain the demanded duration of the impulse response the bandwidth of the several stages should therefore be widened. Although the concept of effective duration of impulse response suggests the presence of distinct axial intervals (based on the speed of ultrasound in tissues), it should be interpreted as the range in which the returned signals contribute predominantly to the detected phase signal at a given time-delay with respect to emission while signals outside this range will contribute to a 
lesser extent. To demonstrate this effect the detected phase signal as induced by a string target (human hair) with a diameter of $45 \mu \mathrm{m}$ perpendicular to the ultrasonic beam (emission frequency $6.1 \mathrm{MHz}$, emission duration $2 / 3$ us equivalent to 0.5 $\mathrm{mm})$ is depicted in fig. 4.3. The phase signal remains present over a longer range than $0.5 \mathrm{~mm}$ due to the smearing of the emitted pulse by the filter constituted by the transducer, receiver and phase detector. The actual axial resolution can be computed from this waveform. To get a practical measure we take as references the $50 \%$ level of the peak value and associate the time-span between both $50 \%$ levels with the axial resolution.

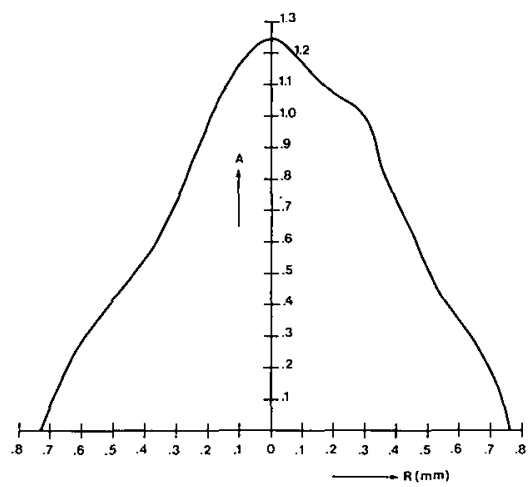

Fig. 4.3: The envelope $A$ of the in quadrature signal as function of depth for a string target (human hair, thickness $45 \mu \mathrm{m}$ ). Duration of emission $2 / 3$ 46. At the -6 dB level the dimension of the sample volume in axial direction is $0.78 \mathrm{~mm}$.

From fig. 4.3 it can be concluded that the axial resolution of this system will be therefore in the order of $0.78 \mathrm{~mm}$. Phase samples taken at these range intervals, as is performed in multi-gate pulsed Doppler systems, represent the phase-signal as function of depth. Increasing the phase sample rate will not contribute more information since then the phase signals will become more and more dependent on each other. On the other hand sampling at intervals greater than the above defined axial resolution will cause loss of spatial information.

In the example as presented above a single string target was used perpendicular to the ultrasonic beam. Targets in a plane perpendicular to the ultrasonic beam can only be distinguished if they are spaced at a distance greater than the local lateral resolution i.e. the local width of the ultrasonic beam. As a 
practical measure for the width we will again take the distance between the $50 \%$ amplitude level with respect to the peak amplitude (center of the ultrasonic beam) assuming rotation symmetry.

The disc shaped volume (Baker, 1973) defined by the axial and lateral resolution is called the sample volume. Improvement of the resolution can be obtained by reducing the dimensions of the sample volume. Since in Doppler systems the sample volume is generally angled with respect to flow direction both dimensions should be in the same order. A further reduction of the smallest dimension of the sample volume (normally the axial resolution) will mainly affect the signal-to-noise ratio while the resolution in the direction of the flow hardly improves.

\subsection{DOPPLER BANDPASS FILTER}

The sampled phase signals contain the Doppler frequencies plus higher frequencies induced by the sampling frequency i.e. the pulse repetition frequency. Moreover, depending on the position of the sample volume low frequency Doppler signals with high amplitude may be present. To suppress the spurious high frequencies and the low frequencies a bandpass filter is employed. The upper cut-off frequency is dictated by the PRF and should be somewhat less than PRF/2. The higher the roll-off for higher frequencies the closer the cut-off frequency can approach $P R F / 2$. The lower cut-off frequency depends on the design and application of the system. If a small sample volume (with respect to the dimensions of the investigated arteries) is employed, vessel wall induced Doppler Erequencies can be avoided by proper positioning of the sample volume. The lower cut-off frequency can then be selected very low. This may, however, cause problems when manouvering the sample volume or when the artery moves with respect to the transducer. Normally, the lower cut-off frequency is therefore comparable with the cutoff frequency of the Doppler filter in CW-systems. The demands 
42

for the roll-off are less stringent since rejection of undesired signals is partly achieved by the position of the sample volume within the artery.

\subsection{DETECTION OF AVERAGE VELOCITY}

The output of the bandpass filters provides the in quadrature Doppler signals as a continuous function of time, originating from a sample volume positioned along the ultrasonic beam. If the dimensions of the sample volume are small with respect to the diameter of the investigated artery it may be anticipated that the blood velocities are in the same order. The Doppler frequency spectrum will be therefore narrow and a zero crossing detector can be used as estimator for the average frequency without introducing a significant bias in the estimator output (section 3.5), provided a good signal-to-noise ratio. For the same reason a frequency splitter to separate positive and negative Doppler frequencies can be omitted. If the sample volume envelopes the artery more or less then the performance of the pulsed Doppler system is comparable with a CW-system. However, the pulsed Doppler system will still show a reduced risk of contamination of the Doppler signals by signals originating from adjacent blood vessels. A frequency splitter and a zero crossing counter for each of the directional audio outputs should then be preferred.

The width of the Doppler frequency spectrum remains finite, even for a flat velocity distribution within the sample volume, due to the transit time effect (Angelsen, 1975; Newhouse, 1976) i.e. the time it takes for a blood particle to pass the sample volume. The transit time effect causes an amplitude modulation of the Doppler signal and, therefore, dispersion of the Doppler frequency spectrum. The bandwidth of the amplitude modulated signal is reciprocal to the transit time. Since the transit time is reciprocal to the velocity the bandwidth will be proportional to the velocity, and, hence, to the average Doppler 
frequency. Therefore, the frequency dispersion due to the transit time affect can be expressed by the fractional bandwidth of the Doppler signal i.e. the effective bandwidth divided by the average Doppler frequency. The amplitude modulation of the Doppler signal depends on the way the blood particles pass through the sample volume (in axial, lateral or oblique direction). Therefore, the envelope of the Doppler signal is a complicated function of the shape of the ultrasound beam in lateral direction and of the envelope of the phase signal as depicted in fig. 4.3. For a blood particle moving with velocity $v$, passing through a sample volume with dimension $L$ (transit length), along the path followed by the blood particle the transit time will be equal to $\mathrm{v} / \mathrm{L}$. Assuming a bandwidth-time product for the observation window equal to 1 , then the effective bandwidth is equal to:

$$
\Delta \mathrm{f}=\mathrm{v} / \mathrm{L}
$$

Hence, the fractional bandwidth can be expressed by:

$$
\Delta \mathrm{f} / \overline{\mathrm{f}}_{\mathrm{D}}=\lambda /(2 \mathrm{~L} \cos \alpha)
$$

where use is made of expression 2.5 and the relation between the emission frequency $\mathrm{E}_{\mathrm{e}}$, the wavelength $\lambda$ and the speed of sound $c$. The spectral broadening depends on the dimension of the sample volume expressed in wavelengths $(L / \lambda)$ and the angle of observation. Changing the angle of observation from $30^{\circ}$ to $60^{\circ}$ will affect the spectral broadening by as much as $70 \%$ (for the same transit length $\mathrm{L})$. For $\lambda=250 \mu \mathrm{m}, \alpha=45^{\circ}$ and $\mathrm{L}=1 \mathrm{~mm}$ the fractional bandwidth will be equal to 0.176. Only frequency dispersion causing a larger fractional bandwidth can be attributed to the observed velocity distribution (Forster, 1977; Angelsen, 1980a). 


\subsection{DISCUSSION}

With a pulsed Doppler system velocity information can be assessed from a small sample volume which can be located anywhere along the ultrasonic beam. The use of small sample volumes avoids contamination of the velocity information by unwanted Doppler signals originating from vessel walls and adjacent blood vessels. To realize a small sample volume the width of the ultrasonic beam should be narrow combined with a large bandwidth of the transducer and receiver stage. If the large transducer bandwidth is achieved by acoustic backing of the transducer then the efficiency of the transducer will be degraded causing a reduced signal-to-noise ratio as compared to CW-systems. Table 4.1 summarizes the relation between various system parameters. The bandwidth of the Doppler signals depends on the velocity dispersion within the sample volume and on the

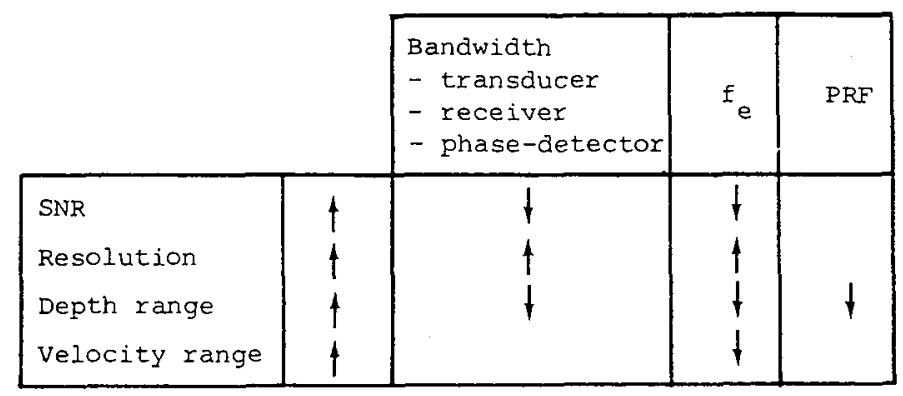

Table 4.1: Relation between various parameters of a pulsed Doppler system.

transit-time effect. This bandwidth will be less than that in $C W$-systems. Because of the narrow frequency distribution detection of the average velocity with a zero crossing detector will introduce a minor bias in the estimated velocity. A drawback of a small sample volume is not only the low signal-tonoise ratio but also the problems encountered in positioning the sample volume at the right location and to maintain it there even if the artery is moving with respect to the transducer. The last mentioned problems can be avoided by employing a multi-gate pulsed Doppler system. 


\section{MULTI-GATE PULSED DOPPLER SYSTEMS}

\subsection{INTRODUCTION}

A single gate pulsed Doppler system can be converted into a multi-gate pulsed Doppler system by duplicating the circuitry needed for the intermediate storage of the phase signals, the filtering of the Doppler signals and the detection of the average velocity for each of the additional channels. Assessment of the velocity distribution in large arteries with relatively small sample volumes requires a large number of processing channels which should be identical to ensure that the outputs of the different channels can be compared with each other. The approach of processing the phase signals by parallel channels will become more and more problematic with an increasing number of channels. Hence, processing of data in serial form by a single circuit should be preferred. This includes storage of the phase samples, filtering and detection of the average velocity for each of the sample volumes.

\subsection{SAMPLED DATA FILTERS}

Filtering of signals, sampled in the time domain but as a continuous function of range, by a single circuit has been introduced in medical ultrasonics a long time ago. It finds its application, for instance, in moving target indication (MTI)

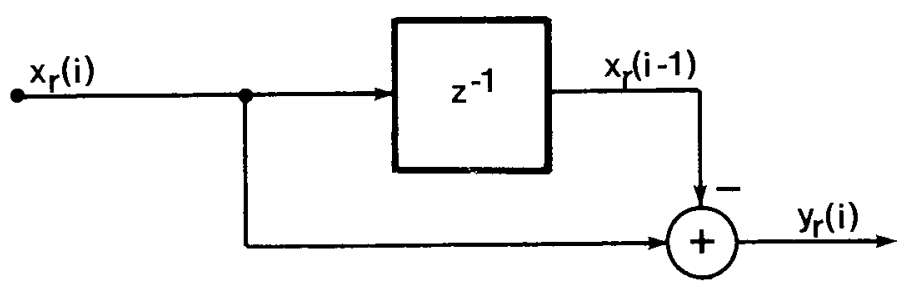

Fig. 5.1 : Blockdiagram for a moving target indication system. The signals $x$ and $y$ are a continuous function of range $r$ and sampled in time by the pulse repetition frequency $(P R F)$. The symbol $z^{-1}$ indicates a delay of $T=1 / P R F$. 
46

systems (Skolnik, 1962) where the received signal is delayed equal to the time interval $T(T=1 / P R F)$ elapsing between two subsequent emissions (Barnes, 1977). As output is taken (fig. 5.1) the difference between the delayed and received signal:

$$
y_{Y}(i)=x_{r}(i)-x_{r}(i-1)
$$

where the index $i$ indicates the $i^{\text {th }}$ sample at $r a n g e r$. Denoting the frequency-spectrum of the sampled signals $x_{r}(i)$ and $y_{r}(i)$ by $x_{r}(\omega T)$ and $Y_{r}(\omega T)$, respectively, then :

$$
Y_{Y}(\omega T)=x_{r}(\omega T)-X_{r}(\omega T) \exp (-j \omega T)
$$

where use is made of the property of the Fourier transformation that a multiplication by $\exp (-j \omega T)$ in the frequency-domain is equivalent to a delay $T$.in the time-domain. Assuming that the signals are properly sampled i.e. that the signal-frequency is less than $\mathrm{PRF} / 2$ then $|\omega \mathrm{T}| \leq \pi$. The relation between input and output is expressed in the frequency-domain by the transfer function $H_{r}(\omega T)$ :

$$
H_{r}(\omega T)=Y_{r}(\omega T) / X_{r}(\omega T)=1-z^{-1}
$$

where $z^{-1}$ stands for $\exp (-j \omega T)$.

The effect of the above signal processing is demonstrated by the substitution for $w \mathrm{~T}$ either $\mathrm{O}$ or $\pi$. Because ${ }_{i} \mathrm{H}_{\mathrm{r}}(0):=0$ and $\mathrm{H}_{\mathrm{r}}(\pi) \mathrm{i}=2$ the circuit acts as a high-pass filter. The cut-off frequency $f_{C}\left(\omega_{C}=2 \pi f_{C}\right)$ can be calculated by equating:

$$
\begin{aligned}
& \left\{H_{r}\left(\omega_{C} T\right):=H_{r}(\pi): / \sqrt{2}\right. \\
& i-\exp \left(-j \omega_{C} T\right):=2 / \sqrt{2} \\
& \omega_{C} T=\pi / 2 \\
& { }_{C}=1 / 4 T=P R F / 4
\end{aligned}
$$

The cut-off frequency is therefore fixed at PRF/4. The bandpass is rather small since $\left|f_{M}\right|<P R F / 2$ (where $E_{M}$ is the maximum 


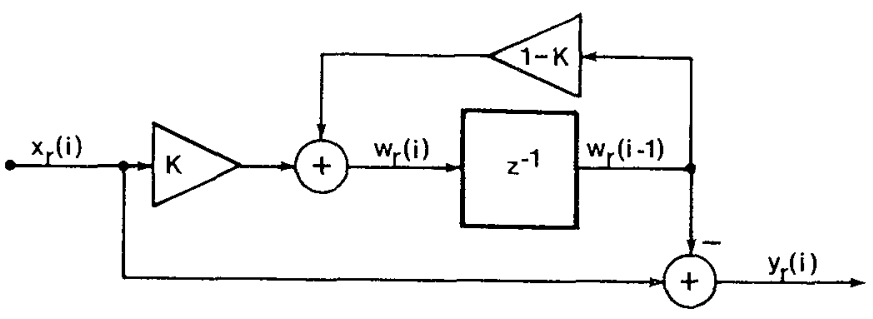

Fig. 5.2 : Blockdiagram for a sampled data high-pass filter of the first order. The filter constant $K$ sets the cut-off frequency with respect to the PRF. The output $w_{p}(i-1)$ and $y_{p}(i)$ is the low-pass and high-pass output of the filter,

frequency) because of the sampling procedure. By introducing a filter constant $K$ (fig. 5.2) cut-off frequencies other than $\mathrm{PRF} / 4$ can be obtained. Denoting the input of the delay-line by ${ }_{r}$ (i) then:

$$
\begin{aligned}
& y_{r}(i)=x_{r}(i)-w_{r}(i-1) \\
& w_{r}(i)=K x_{r}(i)+(1-K) w_{r}(i-1)
\end{aligned}
$$

Converting both expressions into the frequency domain then the transfer function can be expressed as:

$$
\mathrm{H}_{\mathrm{r}}(\omega T)=\mathrm{Y}_{\mathrm{r}}(\omega \mathrm{T}) / \mathrm{X}_{\mathrm{r}}(\omega T)=\left(1-\mathrm{z}^{-1}\right) /\left(1-(1-K) \mathrm{z}^{-1}\right)
$$

Again in this way a high-pass filter results since $\left\{\mathrm{H}_{r}(0) i=0\right.$ and $i_{r}(\pi) i=2 /(2-K)$. The cut-off frequency $f_{C}(K)$ for a first order high-pass filter as function of $K$ can be approximated by (see fig. 5.3a):

$$
\mathrm{E}_{\mathrm{C}}(\mathrm{K})=\mathrm{K} * \mathrm{PRF} / 6
$$

In the same way it can be shown that the transfer function from input to the delayed output $w_{r}(i-1)$ is equal to:

$$
\mathrm{H}_{\mathrm{X}}(\omega \mathrm{T})=\mathrm{K} /\left(1-(1-K) \mathrm{z}^{-1}\right)
$$

This represents a low-pass filter with the same cut-off Ere- 

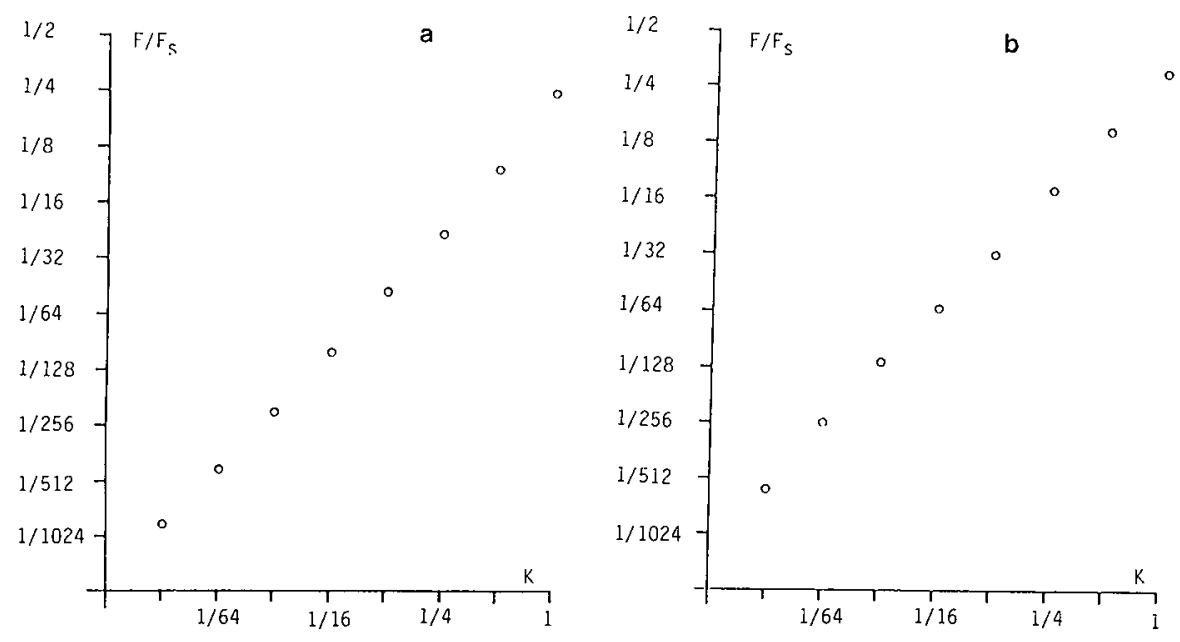

Fig. $5.3:$ Relation between the fractional cut-off frequency $F / F$ (where $F$ is the sampling frequency) and the filter contstant $K$ for a first order sampled data filter highpass filter (left). The cut-off frequency can be approximated by $K^{*} P R F / 6$. Cascading two first order high-pass filters with the same filter constant changes the relation between cut-off frequency and $K$ (right) into $K{ }^{*} P R F / 4$.

quency as the high-pass filter. The system can therefore be used as both a high- pass and low-pass filter of the first order. The response time of the low-pass filter (defined as the reciprocal value of the initial derivative of the output with respect to time as reponse to a unit step at the input) normalized with respect to the sample frequency can be approximated by $1 / K$ (see expression 5.5 ).

The signal-processing in fig. 5.2 involves two multiplications. The number of multiplications can be reduced by reorganizing the system configuration as shown in fig. $5.4 a$.

In most real-time applications the MTI-system as discussed above is used in analog form, i.e. all the signals remain in analog form, requiring an analog delay line to preserve the intermediate information as function of depth. Then the delaytime should be exactly equal to $1 / P R F$ which can be achieved by 

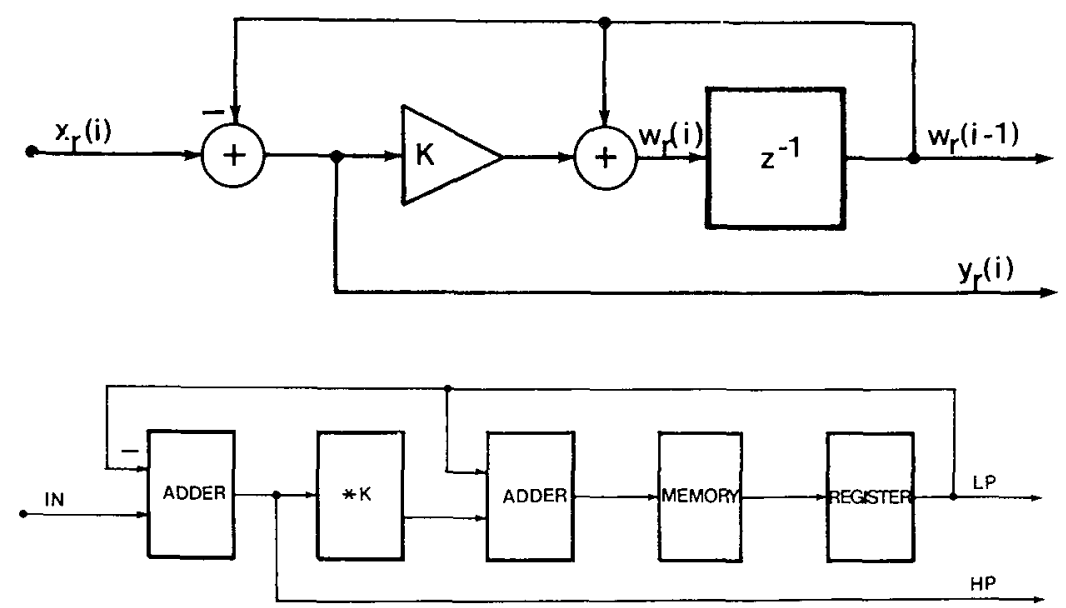

Fig. 5.4 : Reorganized block-diagram for a first order sampled data fizter featuring both a high-pass and low-pass output. Replacing the delay-line by a digital memory and converting the input into digital form results in a digital filter (fig. 5.4b). The number of memory-locations limits the number of signals that can be processed in serial form.

employing a sampled analog delay-line. The signal $w_{r}(i)$ is then sampled in both range and time. More problematic is the dynamic range of the delay-line. Suppose that one would like to maintain a dynamic range of $60 \mathrm{~dB}$, then the dynamic range of the delay-line should be higher due to the filter constant $K$. For example, for $\mathrm{K}=1 / 16$ an extension of at least $24 \mathrm{~dB}$ is required to avoid non-linearities (Oppenheim, 1972). Sampled analog delay-lines with a dynamic range of better than $80 \mathrm{~dB}$ and a feed-through of less than $-80 \mathrm{~dB}$ are hard to find. This problem is not encountered if a digital filter is employed (Ackroyd, 1973). The analog delay-line is replaced by a digital memory and both input and output are represented in digital form (fig. $5.4 \mathrm{~b})$. This requires analog to digital conversion of the phase detector output at range intervals not exceeding the axial resolution to retain all the range information. The sample and hold circuit in the phase detector should keep the detected phase signal stored until analog to digital conversion is completed and then it is ready for acquisition of new data. The 


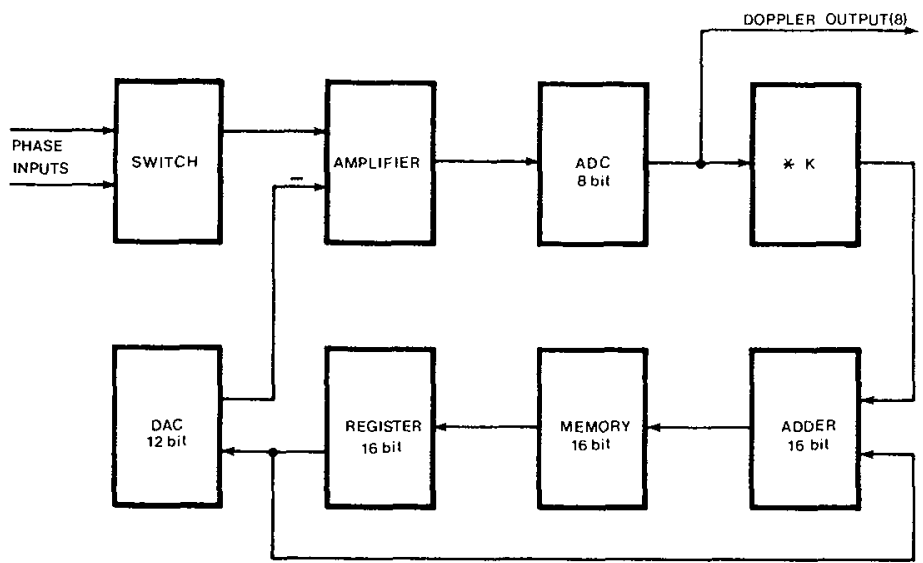

Fig. 5.5 : To reduce the requirements for the dynamic range of the analog-to-digital converter ( $A D C)$ the converter should be incorporated in the digital filter, requiring an additional suming amplifier and digital-to-analog converter $(D A C)$. To process the in quadrature Doppler signals originating from 128 sample volumes the memory should contain 256 memory-locations.

processing rate of the digital filter should be higher than its input rate, i.e. the phase sample rate as function of depth. This requires fast analog to digital conversion for the in quadrature phase outputs. Because not only speed but also the dynamic range of conversion is important, analog to digital conversion will become more and more problematic for increasing phase sample rates. Both problems can be circumvented by converting the high-pass output of the filter (Brandestini, 1976; Brandestini, 1978a) rather than the phase-output itself (see fig. 5.5). Because at this stage the low Doppler Erequencies will be attenuated the required dynamic range for the analog to digital converter (ADC) will be less. Very fast (flash) ADC's can then be used. In the feed-back loop a digital to analog converter (DAC) should be incorporated to enable analog subtraction of both signals. A flash ADC does not require a sample and hold since conversion is performed instantaneously. Because both in quadrature phase outputs should be converted, timemultiplexing is facilitated by maintaining a sample and hold 
circuit in one of the outputs. After the first conversion phase is completed a switch allows the other phase detector output to be processed (fig. 5.5). This multiplexing doubles the required conversion and processing rate but ensures that both phase outputs are processed in the same way. How the implementation of the flash ADC affects the dynamic range of the Doppler signal and how a high processing rate can still be achieved will be discussed in the sections 5.3 and 5.4 .

The number of phase samples in quadrature that can be taken as function of depth is limited by the number of memory locations in the digital filter. One should keep in mind that a long observation range (i.e. number of phase samples times the sample distance) will limit the PRF and therefore the maximal Doppler frequency range. On the other hand the ability to focus on a short range interval would be very helpful to visualize detail in the assessed velocity-distribution. This is achieved most easily by making the time-delay with respect to emission at which the first phase-sample is taken flexible in combination with a variable number of phase-samples.

\subsection{THE DYNAMIC RANGE OF THE DIGITAL FILTER}

As stated before the cut-off frequency of the digital filter is for a given filter constant $K$ proportional to the PRF. Taking $\mathrm{K}=1 / 16$ and $\mathrm{a} P R F$ of $20 \mathrm{kHz}$ will result in a cut-off frequency (expression 5.7 ) of $K^{*} P R F / 6=208 \mathrm{~Hz}$. Selecting filter constants being integral powers of 2 i.e. $k=2^{-n}$ is favourable because multiplication by $2^{-n}$ can be easily realized by hardwired shifting which does not require processing time. Due to the shifting the word length at the input of the memory should be extended to reauce the effect of truncation. It can be shown (Oppenheim, 1972) that the noise induced by truncation will be the same as the digitizer noise if the word length is extended with $\mathrm{n}$ bits for $\mathrm{K}=2^{-\mathrm{n}}$. The digitizer accuracy is $1 / 2 \mathrm{LSB}$ (least significant bit) and independent of the truncation noise. The 
52

combined noise power will be therefore $1 / 6$ LSB $^{2}$ assuming a rectangular amplitude distribution for both noise sources.

The limited dynamic range of the low-pass output of the digital filter will reduce the ability to cancel the low-frequency Doppler signals and, therefore the overall dynamic range. Since the signal-to-noise ratio largely affects the velocity estimator output (see section 3.5) the signal-to-noise level should not be restricted by the dynamic range of the filter. we will allow for the filtered Doppler signals a range of at least $30 \mathrm{~dB}$. The Doppler signals originating from blood particles can be obscured by low frequency Doppler signals induced by tissue interfaces, like vessel walls, by some $30 \mathrm{~dB}$. Allowing an additional $12 \mathrm{~dB}$ for the from person to person varying level of the returned signal and of the filter noise (due to conversion accuracy and truncation) a dynamic range of $72 \mathrm{~dB}$, equivalent to a 12-bits resolution, is required for the low-pass output of the digital filter (Hoeks, 1981). Then a 12-bits DAC should be used in the analog feed-back loop. The dynamic range as function of frequency at the high-pass output (the input of the $A D C$ ) depends on the selected cut-off frequen-

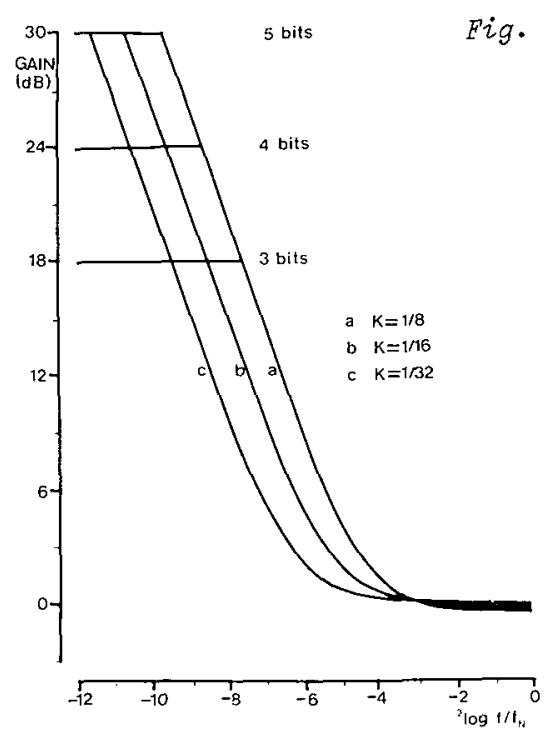

5.6: Frequency transfer characteristic for the hybrid filter as depicted in fig. 5.5 for 3 values of the filterconstant $K$ and for 3 dynamic ranges of the employed $A D C$, in the figure indicated by the difference in the number of bits of the DAC and the $A D C$. Is this number Low (e.g. 3 bits) then the dynamic range for Low frequency signals will be extended by only $18 \mathrm{~dB}$. 
cy in combination with the overall frequency content of the Doppler signal. The dynamic range of the ADC should exceed the anticipated dynamic range of the Doppler signal at these frequencies. A $36 \mathrm{~dB}$ range and hence a 6 bits $A D C$ will be sufficient but the situation becomes more complicated for Doppler frequencies just below the cut-off frequency of the high-pass filter. In this frequency range we should also take into consideration the anticipated frequency content of the unfiltered Doppler signals. In fig. 5.6 the frequency spectrum that can be processed by this hybrid digital filter is depicted for $k=2^{-n}$ where $n=3,4$, or 5 and for the selected difference in the dynamic range of the $A D C$ and the $\operatorname{DAC}(3,4$, or 5 bits). As can be expected the dynamic range of the signal as function of the frequency falls from the maximum range set by the 12-bits DAC to the range dictated by the accuracy of the ADC. To decide about the resolution of the ADC and the cut-off frequency of the filter we do need information about the frequency content of Doppler signals induced by vessel wall motion. Let us consider an artery with a diameter of $6 \mathrm{~mm}$ and a relative change in diameter in the order of 10 o during a cardiac cycle (Arndt, 1968; wetterer, 1968). This change in diameter is reached within $100 \mathrm{~ms}$ in the early systolic phase, indicating a peak velocity of the wall in the order of $3 \mathrm{~mm} / \mathrm{s}$, assuming that both the anterior and posterior wall are contributing equally to the increase in vessel diameter. If the ultrasound beam is aimed perpendicular to the vessel wall, then the largest signal amplitude is returned towards the transducer. The Doppler frequency induced by the vessel wall motion will be $25 \mathrm{~Hz}$ for a 6 $\mathrm{MHz}$ Doppler system. Reducing the angle between ultrasound beam and vessel (as in normal operation) will give a significant reduction in the level of the acoustic signal returned towards the transducer while the blood velocity in the direction of the transducer and, therefore, the Doppler frequency will increase. Considering the situation where the angle between ultrasound beam and vessel wall is $30^{\circ}$ (Doppler frequencies of up to 50 $\mathrm{Hz}$ ) and assuming a reduction in signal level of $12 \mathrm{~dB}$ we can use fig. 5.6 to select the dynamic range of the $A D C$ and the 
54

filter constant $K$ for a pre-selected PRF of $20 \mathrm{kHz}$. A Doppler frequency range of $50 \mathrm{~Hz}$ is then equivalent to a frequency of $1 / 200$ of the maximum Doppler frequency range. As expected a 9 bits ADC allows maximum flexibility in the selection of the cut-off frequency while an 8-bits ADC will limit the choice of the filterconstant to minimal $k=1 / 16$ (cut-off frequency 208 $\mathrm{Hz}$ ). If the $\mathrm{PRE}$ is reduced to $10 \mathrm{kHz}$ then either $\mathrm{K}=1 / 4$ or $\mathrm{K}=1 / 8$ is allowed to achieve a cut-off frequency beyond $208 \mathrm{~Hz}$.

The above reasoning is based upon assumptions about signal level and frequencies induced by moving vessel walls. In practice the conditions might be more favourable because of the angle of observation and the amplitude of the low-frequency Doppler signals. Since an 8-bits ADC flash converter is commercially available we used this type of converter in combination with a selectable. filter constant of either $K=1 / 8$ or $K=1 / 16$. This allows for a flexible adjustment to changes in recording conditions (a lower PRF for larger penetration depths) or spectral composition of the Doppler signal.

\subsection{TIMING CONSIDERATIONS FOR THE DIGITAL FILTER}

With a duration of emission of $2 / 3$ us the minimal axial resolution is in the order of $0.5 \mathrm{~mm}$ (see section 4.5 ). Therefore, the axial sampling distance should be maximally $0.5 \mathrm{~mm}$ to preserve all the spatial information, requiring an axial phase sample rate of $1.5 \mathrm{MHz}$. Time-multiplexing both phase-outputs at the input of the hybrid filter doubles the minimal conversion rate of the $A D C$ and the processing rate of the filter to $3 \mathrm{MHz}$. This leaves $1 / 3 \mathrm{\mu s}$ for the DAC, the analog switch and settling time of the summing amplifier. This requires a high-speed ana$\log$ switch and a high-speed amplifier with a high slew-rate. To ensure that phase samples are taken at the same moment one phase-output is delayed with $1 / 3$ us requiring a sample and hold. Simultaneous with the conversion command new data is fed into the input of the 12-bits DAC and the filter input switches 
to the second phase output. The ADC used was an 8-bits converter featuring a maximal conversion rate of $30 \mathrm{MHz}$. This converter has a pipe-lined operation, i.e. conversion is performed in 2 steps. In the first step the status of the 255 internal comparators is strobed into an internal register while the second step involves the coding to an 8-bits output word. Both actions are performed simultaneously and are sequenced with the conversion command, i.e. at a conversion command the present converter input is strobed while the coded result of the previous input appears at the output. Therefore, one additional (dummy) conversion is required command in a series of conversions. The digital processing includes digital multiplication with the filter constant $K$, digital addition and storage of the intermediate data in the memory (random access memory). Thereafter the data for the next sample point should be retrieved from the memory. To retain data during the write/read cycle of the memory, the memory output is temporary stored in a register. Scaling, addition and write procedure take less than 200 nsec, allowing 80 nsec for the access time of the memory and the set-up time of the registers. Scaling of data can be performed hardwired if only one cut-off frequency relative to the PRF is needed, but we included a digital switch to allow a change in filter constant $(K=1 / 8$ and $K=1 / 16)$ under front-panel control.

The hybrid filter consists of an analog and digital feedback loop. Care should be taken that intermediate data belonging to a specific sample point remain synchronous. The analog feed-back loop requires that data to the DAC is presented one conversion cycle in advance. The ADC introduces a delay of another conversion cycle because of its pipe-lined structure. To compensate for this, a delay of 2 conversion cycles should be inserted in the digital feed-back loop. This is achieved by a cascade of two 16-bits registers (fig. 5.7). Since data is read from the memory 2 conversion cycles before the intermediate result can be stored, the addressing of the random access memory will become complicated and too time consuming if one wishes to maintain a one-to-one relationship between address and 


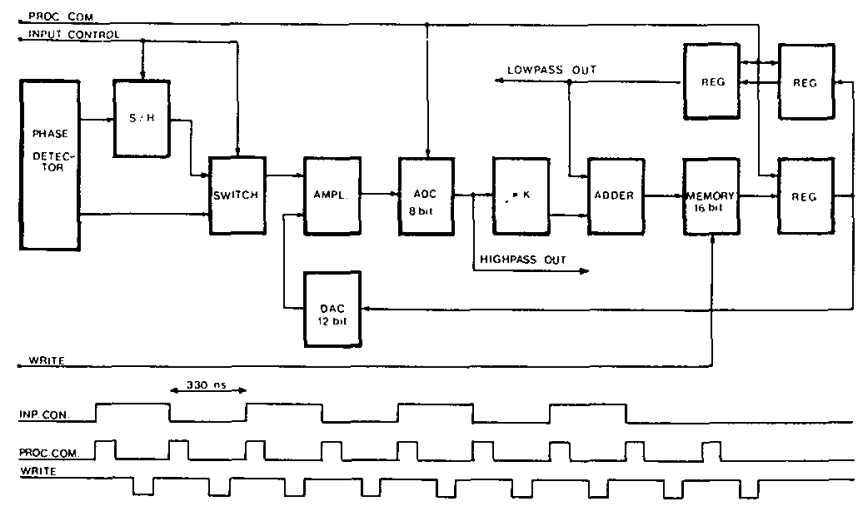

Fig. 5.7 : Complete block-diagram including timing diagram for a hybride sampled data filter of the first order. The cascade of data-registers compensates for the delay in the analog feedback loop. The processing rate for the system is $3 \mathrm{MHz}$.

number of sample points to be processed. Because of timing considerations we preferred the addressing of the memory in a scroll mode. This requires that the number of memory locations is fixed to twice (because of in quadrature data processing) the total number of active sample points minus two (because the additional registers, to compensate for the delay in the analog feed-back loop, acts as extension of the memory) plus one for the dummy conversion. A synchronous divide by $2 \mathrm{~N}-1$ counter, where $\mathrm{N}$ equals the total number of sample points, is used to generate the address for the random access memory. The writecommand for the memory is used to clock the address counter. Using a RAM with 256 locations allows for the processing of maximal 128 sample points. The approach as given above makes it easy to adjust $N$ to the desired range of observation. Any $N$ which is an integral multiple of 8 up to a maximum of 128 can be selected under front panel control.

To increase the roll-off of the high-pass filter to $12 \mathrm{~dB}$ per octave a second high-pass filter is cascaded. Cascading two filters of the first order will affect the cut-off frequency of 


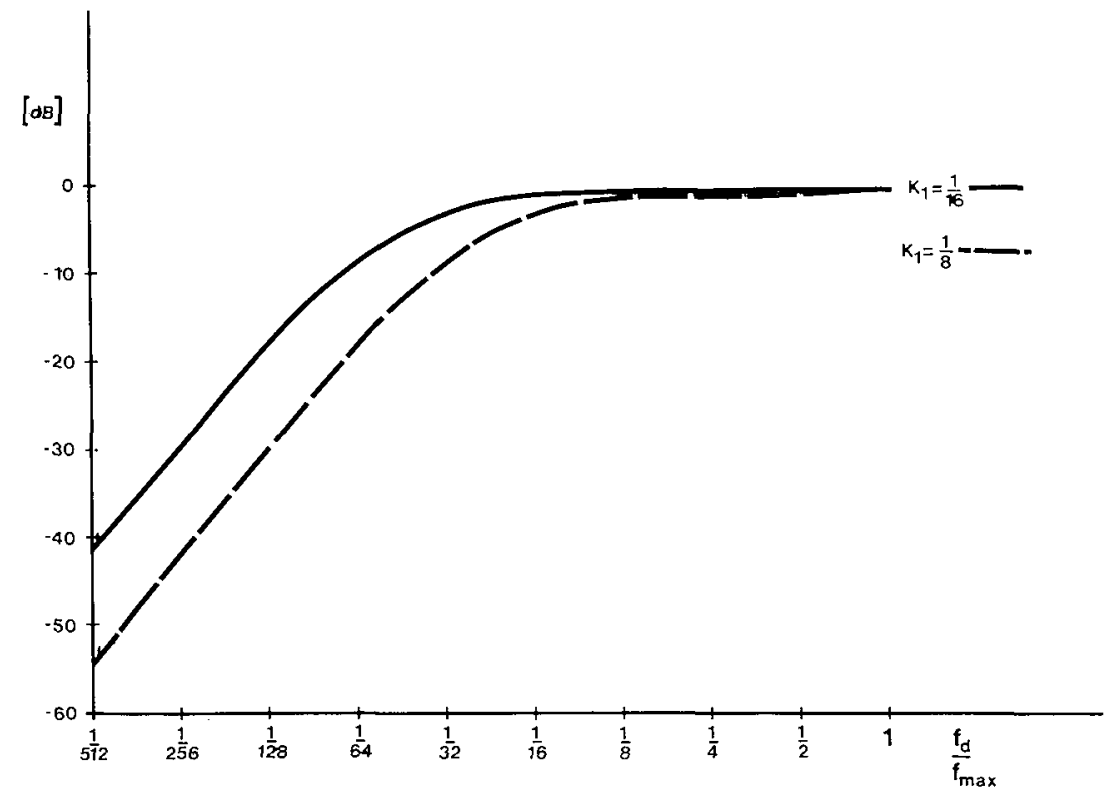

Fig. 5.8 : Frequency transfer characteristic for a cascade of two first order digital filters for two values of the filter-constant $K$.

the filter. If both filters employ the same filter constant $K$, then the cut-off frequency as function of $K$ can be approximated by (see fig. $5.3 b$ ) :

$$
\mathrm{f}_{\mathrm{C}}(\mathrm{K})=\mathrm{K} * \mathrm{PRF} / 4
$$

This filter completely consists of digital circuitry. Since for this filter no additional delays are required, a one-to-one relation between memory address and sample point can be maintained. The address counter is reset to zero at the beginning of the processing cycle. The cut-off frequency (and therefore K) of the cascaded high-pass filter is the same as that of the first filter. The overall transfer characteristic for $K=1 / 8$ and $1 / 16$ is depicted in fig. 5.8 where the frequency axis is scaled with respect to the maximal frequency i.e. PRF/2. The cut-off frequencies in $\mathrm{Hz}$ are listed in table 5.1 for both $K=I / 8$ and $\mathrm{K}=1 / 16$ and for $\mathrm{PRF}^{\prime} \mathrm{S}$ of $19 \mathrm{kHz}$ and $9.5 \mathrm{kHz}$. 
Table 5.1: Cut-off frequency (Hz) for a cascade of two high-pass filters of the first order as function of the filter constant $K$ and pulse repetition frequency (PRE).

\begin{tabular}{|l|c|c|}
\cline { 2 - 3 } \multicolumn{1}{c|}{} & \multicolumn{2}{c|}{ PRF } \\
\cline { 2 - 3 } \multicolumn{1}{c|}{} & $19 \mathrm{kHz}$ & $9.5 \mathrm{kHz}$ \\
\hline $\mathrm{K}=1 / 8$ & 625 & 303 \\
$\mathrm{~K}=1 / 16$ & 312 & 152 \\
\hline
\end{tabular}

At the high-pass output of the second filter the processed value appears in serial form for the selected number of sample points. For audio evaluation one of the in quadrature high-pass filtered Doppler signals of a pre-selected sample point is gated to an 8-bits DAC followed by a second order analog lowpass filter to suppress frequencies above PRF/2. Since there are two options for the PRF two analog filters with a cut-off frequency of $P R E / 2$ are necessary.

\subsection{DETECTION OF AVERAGE FREQUENCY}

A zero crossing detector provides a simple method to estimate the average Doppler frequency (see section 3.5 and section 4.7). For a signal with a non-zero bandwidth the output of this frequency estimator is biased. The error in the output increases with the fractional bandwidth of the Doppler signal. Since the anticipated velocity dispersion within a small sample volume is minimal the fractional bandwidth of the assessed Doppler signal will be small. Under this condition the error in the output due to the method of detection can be neglected.

To obtain the average frequency of the filtered Doppler signal originating from a sample volume (representing the average velocity in that sample volume with respect to the transaucer) with a zero crossing detector (see section 3.5) two actions should be taken. The first action involves the detection of a zero crossing including the sign, while in the second action frequency components are removed which are of non-physiological origin and can be attributed to the estimation procedure. 
To detect a zero crossing in a time-sampled system we need the level (positive or negative) of the signal with respect to a pre-set threshold in two successive processing cycles. This requires a memory to retain the level (Brandestini, 1976; Brandestini, 1978a; Hoeks, 1981). A zero crossing is detected if the present level with respect to the threshold deviates from the previous one. The threshold should be pre-adjusted such that in the absence of true Doppler signals no crossings, induced by system noise, are detected. A hysteresis is introduced by keeping the polarity of the threshold opposite to the polarity of the signal with respect to the threshold. The sign of the threshold can be derived by taking into account the polarity of the second in quadrature Doppler signal at the occurrence of a threshold crossing in the first one. This procedure will be only unambiguous if the polarities of both in quadrature Doppler signals do not change coincidently. However, for Doppler signals with frequencies beyond PRF/4! a simultaneous change in the polarity of both in quadrature signals can be observed. If that happens another source of information is needed to decide about the direction of the velocity in the sample volume and to circumvent the polarity ambiguity. Assuming that the PRF is sufficiently high (no frequency ambiguity), then it is unlikely that the velocity will change direction within two processing cycles. Then the output of the low-pass filter following the zero crossing detector will provide the necessary information about the direction of the velocity. If polarity ambiguity is detected (the polarity of both in quadrature Doppler signals have changed coincidently), then the assigned direction will be given by the sign of the output of the low-pass filter used to compute a running time average of the density of zero crossings.

If a digital filter is employed to obtain the low-pass output this filter should be activated in each processing cycle. Its input would be either plus or minus one if a zero crossing has been detected otherwise it will be zero. The cut-off frequency is then dependent on the PRE for a given filter con- 


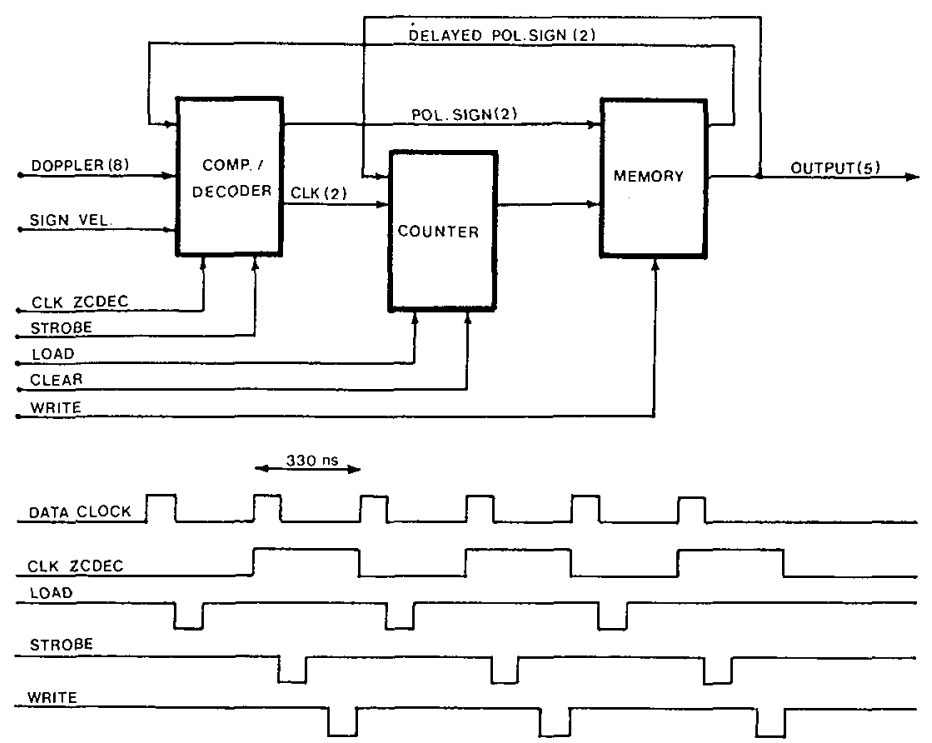

Fig. 5.9 : Block-diagram of the zero crossing decoder. Based on the delayed polarity information of the in quadrature Doppler signals, the present values of the Doppler signal and the sign of the time-average of the zero crossings the zero crossing counter is activated accordingly. The memory enables the serial processing of Doppler signals originating from adjacent sample volumes.

stant. To circumvent this problem the detection and processing of zero crossings is executed in two steps. The zero crossing detector contains an up/down counter, followed by a memory to retain intermediate results. The output of the memory is transferred to the low-pass filter at a fixed rate of $1200 \mathrm{~Hz}$. For a PRF of $19 \mathrm{kHz}$ then a maximum of 16 zero crossings can be detected. In the memory of the zero crossing counter 2 bits are reserved to retain the polarity of the in quadrature Dopoler signals. In fig. 5.9 the basic configuration of the zero crossing counter is depicted. Since the in quadrature Doppler signals arrive at the threshold comparator in a sequential form a serial to parallel conversion of the detected polarities is necessary, requiring a Elip-flop activated by CLK ZCDEC (fig. 5.9). If both comparisons are completed the decoder is strobed, resulting in either a clock up or clock down command for the 
zero crossing detector if a crossing has been detected. The counter is preloaded with the intermediate result of each activated sample volume, temporarily stored in the memory (RAM). At a fixed processing rate of $1200 \mathrm{~Hz}$ the memory output is transferred to the low-pass filter while the counter is cleared initiating a new counting cycle. The number of memory locations is 128 .

In peripheral vessels the frequency content of the velocity waveform is generally limited to $10-20 \mathrm{~Hz}$ (McDonald, 1974; Farrar, 1979; Skidmore, 1980). The cut-off frequency of the lowpass filter should be, therefore, in the same order. Employing a digital filter of the first order (fig. 5.10) the filter-constant $K$ should be equal to $1 / 16$ to obtain a cut-off frequency
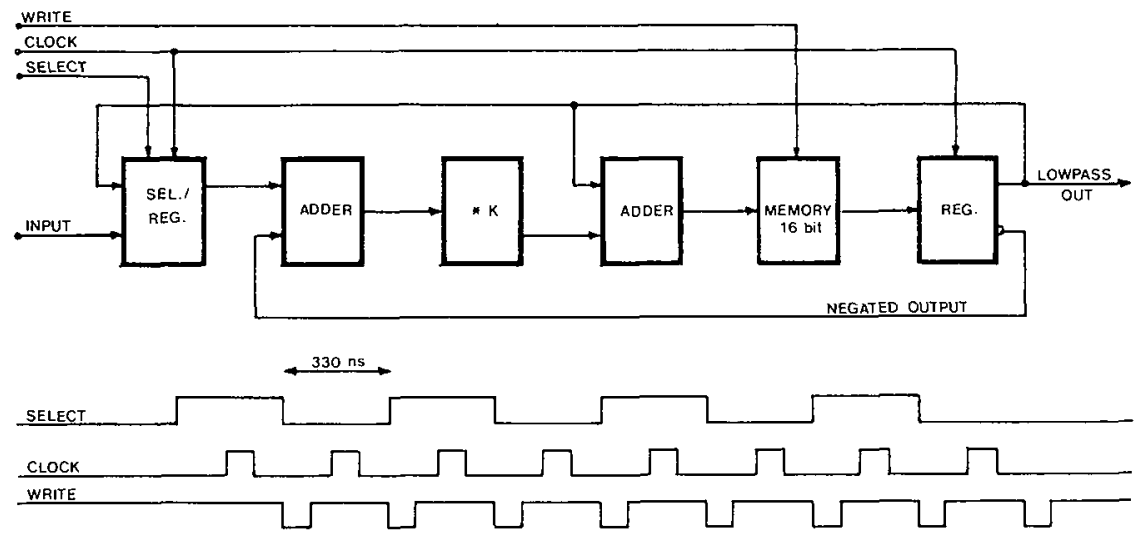

Fig. 5.10: The output of the zero crossing counter as depicted in fig. 5.9 is filtered by a cascade of two low-pass filters of the first order. Cascading is achieved by the incorporation of an additional register/selector.

in the order of $12.5 \mathrm{~Hz}$ for a processing rate of $1200 \mathrm{~Hz}$ (expr. 5.7). To allow for a higher cut-off frequency a switch is included enabling, under front panel control, the selection of either $K=1 / 16$ (cut-off frequency $12.5 \mathrm{~Hz}$ ) or $\mathrm{K}=1 / 8$ (cut-off frequency $25 \mathrm{~Hz}$ ). To increase the roll-off of the low-pass filter to $12 \mathrm{aB} /$ octave two digital filters of the first order are cascaded. This can be achieved without much additional circuitry by a feed-back of the first output to the input and 
62

doubling the available memory locations to 256. At a rate of $1200 \mathrm{~Hz}$ the velocity distribution over the observation range appears in digital format at the output of the filter. Employing an 8-bits DAC this signal is converted into analog form and displayed on a CRT. The time-base of the CRT is automatically adjusted for the number of activated sample volumes, i.e. the screen length is fully utilized independent of the selected number of sample volumes. The sample volumes can be distinguished by blanking the velocity display at the transition of sample volumes for a duration of 160 nsec. This blanking procedure overrides also the transients induced by the DAC.

From the same sample volume as selected for audio evaluation the velocity as a continuous function of time is made available by employing a second DAC with internal register which is only updated if the velocity signal of the selected sample volume appears at its input. On the CRT the selected sample volume is indicated by intensifying the CRT-beam for the duration its velocity signal is displayed. This marker facilitates easy selection of the sample volume for audio output and provides information about the site of investigation (e.g. midstream or near the vessel wall).

\subsection{DETECTION OF SIGNAL AMPLITUDE}

As stated before the detected in quadrature Doppler signals may originate from blood particles as well as from stationary or slowly moving tissue interfaces. Doppler signals induced by erythrocytes are low in amplitude but, generally, higher in frequency than Doppler signals received from tissue interfaces. This distinction in amplitude and frequency can readily be visualized by calculating the envelope of the Doppler signal for several frequency ranges. In the system as presented so far two frequency ranges can be distinguished: the low-pass signal used in the analog feedback loop and the high-pass output of the Doppler filter. The low-pass signal contains information 
about both stationary and slowly moving tissue interfaces. We may assume that the low-pass output does not contain frequencies above the cut-off frequency because of the filtering characteristic and because the higher frequency components have a significantly lower amplitude. Moving structures can be distinguished from stationary interfaces by adding a high-pass filter with a low cut-off frequency (Olsen, 1977). The selection of this cut-off frequency is rather arbitrary since movements relative to the transducer are detected. Therefore, one is never certain whether the tissue interface or the transaucer, due to a change in applied pressure, is moving. We, therefore, made the cut-off frequency of the digital highpass filter selectable at either 5 or $10 \mathrm{~Hz}$. The input rate of the filter to separate slowly moving (vessel walls) from stationary tissue interfaces is fixed at $1200 \mathrm{~Hz}$ to avoid small filter constants which would require a large number of additional bits. Presently the filter is only of the first order. With the addition of the filter for vessel wall movements the amplitude of Doppler signals in 3 Erequency ranges can be distinguished (fig. 5.11):

Fig. 5.11: Anticipated amplitude of the received signal as function of depth for three frequency-intervals, below $300 \mathrm{~Hz} \mathrm{(a),} \mathrm{between} 10-$ $300 \mathrm{~Hz}$ (b) and beyond the cut-off frequency of the Doppler high-pass filter (c) after appropriate amplification. Fig. $5.11 b$ indicates the position of moving $(\mathrm{e} . \mathrm{g}$. vessel wailts) structures while fig. 5.11c gives the position of the vessel Iumen.
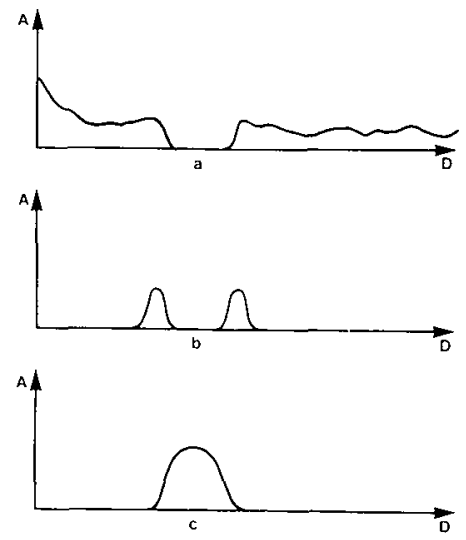

a. in the frequency range from zero to the cut-off frequency of the Doppler filter (echo)

b. in the frequency range from either 5 or $10 \mathrm{~Hz}$ to the cut-off frequency of the Doppler filter (vessel wall) 
64

c. in the frequency range from the cut-off frequency of the Doppler filter up to PRF/2 (Doppler).

Denoting the in quadrature Dopplersignals by $A(t) \cos \left({ }_{D} t\right.$ and $A(t) \sin \omega_{D} t$ the amplitude $A(t)$ can be computed following the algorithm:

$$
A(t)=\left(A^{2}(t) * \cos ^{2} \omega_{D} t+A^{2}(t) * \sin ^{2} \omega_{D} t\right)^{\frac{1}{2}}
$$

Fig. 5.12 gives the basic configuration of the circuit employed for the detection of the instantaneous amplitude of the Doppler signals in the various frequency ranges. Since both in quadrature Doppler signals arrive at the input of the circuit in serial form, a register is inserted to accomplish serial to parallel conversion. The instantaneous amplitude is for each of the frequency ranges computed at a fixed processing rate of $1200 \mathrm{~Hz}$. Therefore, three subsequent processing cycles (the data processing after emission of a ultrasound pulse) are required to detect the amplitude in the activated sample volumes. For a PRF of $19 \mathrm{kHz}$ the amplitude detector remains idle for the remaining 13 processing cycles. The amplitude distribution is displayed at the bottom of the CRT used for the display of the velocity distribution along the ultrasonic beam, employing the same digital to analog converter. This requires a common data bus for both the velocity and amplitude information.

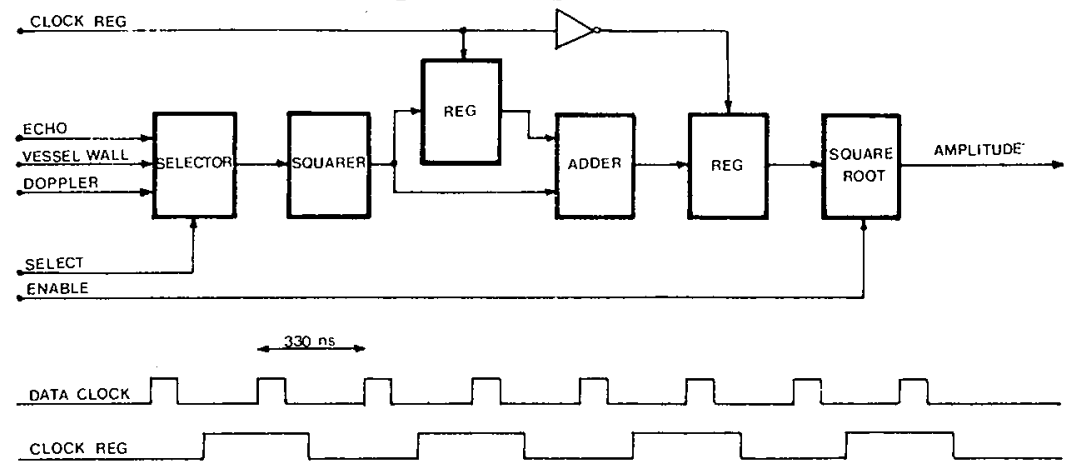

Fig. 5.12: Block-diagram of the envelope-detector based on the square root of the sum of squares of the in quadrature Doppler signals. 


\subsection{DETECTION OF RELATIVE CHANGE IN VESSEL DIAMETER}

The reflected and backscattered acoustic signal contains information about movements of the arterial walls. If this information can be retrieved it would be possible to track transcutaneously changes in vessel diameter caused by pressure cnanges during the cardiac cycle. It has been demonstrated (Hokanson, 1972; Uematsu, 1981) that an echo system equipped with a phase locked loop is capable to provide this information. However, the phase locked loop may fail if the echoes originating from the anterior and posterior walls are obscured by stationary echoes. This problem is circumvented if a Doppler system designed to process low-frequency signals is employed. A CW-system (Olsen, 1977) can be converted into a detector of vessel wall movements by reducing considerably the cut-off frequency of the Doppler filters. However, no information is obtained about the origin of the signals and assumptions have to be made about the contribution of both vessel walls to the detected signals. Application of pulsed Doppler system circumvent this limitation. Wildi (wildi, 1980) proposed a vessel wall tracking system based on the envelope of the high frequency Doppler signals originating from 4 sample volumes. Because of its nature the envelope of the Doppler signal will fluctuate rapialy. Moreover, the cut-off frequency of the Doppler filter will atfect the ratio of the envelope of the Doppler signals originating from both sample volumes coinciding with the vessel walls with respect to the envelope of the Doppler signals originating from both sample volumes positioned within the vessel. Automatic positioning of the sample volumes coinciding with the anterior and posterior wall on the condition that the above defined ratio is maintained (wildi, 1980) creates some tecnnical problems. In our multi-gate pulsed Doppler system the Doppler signal in three different frequency ranges is available (see section 5.6). On-line detection of changes in arterial diameter is possible by processing the low frequency signals originating Erom sample volumes coinciding with the anterior and posterior vessel walls. Identification of the vessel walls is enablea by 
66

the display of the instantaneous amplitude of the Doppler signals with frequencies in the range from 5 to $300 \mathrm{~Hz}$ as function of $r$ ange (see section 5.6). This indentification is supported by the displayed velocity distribution along the ultrasound beam. Because of the finite size of the sample volume and the angle of observation (fig. 5.13) the vessel wall echoes will

Fig. 5.13: Schematic representation for the position of the sample volumes with respect to the vessel. Based on the presence of lowfrequency signals with high amplitude the diameter $D_{b}$ of the artery as measured along the ultrasound beam can be estimated. A change in diameter from $D_{d}$ (diastole) to $D$ (systole) can be detected based on the low frequency Doppler signals originating from the sample volumes coinciding with the vessel walls.

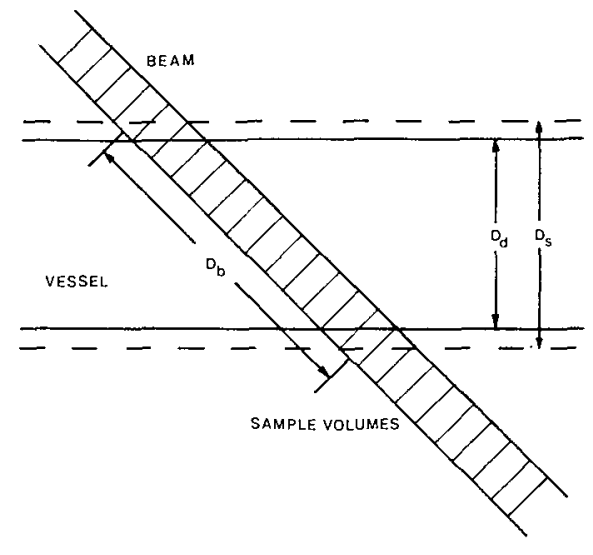

show up in several adjacent sample volumes. Visual evaluation of the amplitude distribution enables the selection of the two sample volumes coinciding with the main position of the anterior and posterior vessel walls. The vessel diameter as measured along the ultrasound beam deviates from the true aiameter because of the observation angle and can be estimated with an accuracy of only $0.5 \mathrm{~mm}$. This is insufficient to detect aiameter changes during a cardiac cycle. But within a sample volume a change in position of a reflector can be sensed more accurately because of the change in phase between received signal and the in quadrature reference signals. For one period of tne Doppler signal equivalent to an accumulated change in phase of $2 \pi$ the reflector has to move within the sample volume $\lambda / 2$. with a zero crossing detector changes in phase of $\pi / 2$ including sign can be detected equivalent to a displacement of $\lambda / 8$ representing an accuracy of $\lambda / 16$. For a $6 \mathrm{MHz}$ pulsed Doppler system tne wavelength in tissue equals $250 \mu \mathrm{m}$ giving rise to high relia- 
bility of the detected displacement $(16 \mathrm{~m})$. Accumulating the phase changes, after apropriate scaling, will result in the displacement of the vessel wall as function of time. Performing this operation on both the anterior and posterior wall and taking the difference will give the change in diameter as function of time as measured along the ultrasound beam. This procedure automatically corrects for displacements of the artery as a whole provided that the movement is along the ultrasouna beam. The detected change will be dependent on the angle of observation but the diameter of the vessel as measured along the ultrasound beam will change likewise. Taking the ratio of both corrects for the angle of insonation and results in a relative change in diameter which is expressed in a percentage of the diameter of the investigated artery.

For this purpose the in quadrature low frequency Doppler signals originating from both sample volumes coinciaing with the anterior and posterior wall of the blood vessel are gated to a zero crossing detector. The selected sample volumes are indicated on the CRT with a marker while selection of the anterior wall and the distance between anterior and posterior wall can be made under cursor control. Because the processing rate of the zero crossing detector is $1200 \mathrm{~Hz}$ (the same as usea for the high-pass filter for the detection of the position of tne vessel walls) neither polarity ambiguity nor frequency ambiguity is anticipated. The polarity of both in quadrature Doppier signals of both sample volumes are stored in an 8 bit snift register (fig. 5.14). At the end of a processing cycle the shift register contains the present polarity bits as well the delayed polarity bits. The zero crossing decoder is then strobed and the up/down counter activated accoraing to the detected change in phase between the Doppler signals of both selected sample volumes. The digital divider ensures proper scaling of the accumulated change in diameter with respect to the observed anterior/posterior wall distance (APD). Because the detector for the relative change in diameter will drift when positioning the ultrasound beam, the counter is reset synchronously with 


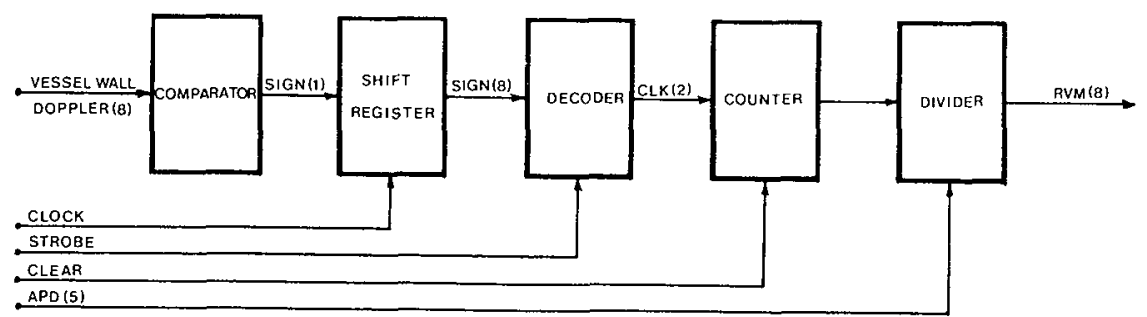

Fig. 5.14: Block-diagram for the detector of the relative change in vessel diameter. The polarities of the in quadrature Doppler signals originating from the selected sample volumes are temporarily stored in a shift register. Via a decoder the zero crossing counter is activated accordingly. The counter is reset synchronously with the ECG. The relative vessel movements (RVM) are obtained by taking the ratio of the counter output and the distance between anterior and posterior vessel wall $(A P D)$.

the R-top of the ECG. Drift may be also induced if the artery as a whole is moving during the cardiac cycle in a lateral direction with respect to the ultrasound beam. The detection of vessel diameter changes simultaneously with the detection of the velocity distribution along the ultrasonic beam, therefore, requires some skill of the operator to aim the ultrasound beam. The operating errors will become more prominent if the vessel diameter gets smaller (in the order of 4 times the diameter of the employed ultrasound beam).

In the theoretical evaluation of the performance of the system to detect relative changes in artery diameter during the cardiac cycle we have to consider the accuracy with which the diameter and the change in phase can be detectea. As stated before arterial wall motions can be detected within a range or $\lambda / 8$. Since the error made in this detection is due to quantization we may assume a rectangular probability density iunction for this error. Taking the movements of the anterior wall witn respect to the posterior wall will, hencefortn, result in a trangular probability density function with $\lambda / 4$ as base. ine associated RMS-error then equals: 


$$
\begin{aligned}
\sigma_{M} & =\lambda / 4 \sqrt{24} \\
& =12.75 \mu \mathrm{m} \text { for } \lambda=250 \mu \mathrm{m}
\end{aligned}
$$

Similarly, if the position of the vessel wall can be estimated within a range of $1 \mathrm{~mm}$ with a rectangular probability density function, then the RMS-error in the measurement of the vessel diameter along the ultrasound beam will be:

$$
{ }^{\sigma} D=2 / \sqrt{24}=0.41 \mathrm{~mm}
$$

Denoting the diameter of the vessel with $D_{b}$ and the relative change in diameter with $\Delta D_{b}$ (as measured along the ultrasound beam) then the relative change in diameter $D_{R}$ is:

$$
D_{R}=100 \Delta D_{b} / D_{b}
$$

Employing a Taylor series expansion of the first order for

\begin{tabular}{|c|c|c|c|c|c|c|c|c|}
\hline & & \multicolumn{7}{|c|}{$D_{b}(m n)$} \\
\hline & & 4 & 6 & 8 & 10 & 12 & 14 & 16 \\
\hline \multirow{6}{*}{$\Delta D_{b}(8)$} & 4 & 0.52 & 0.35 & 0.26 & 0.21 & 0.17 & 0.15 & 0.13 \\
\hline & 6 & 0.69 & 0.46 & 0.35 & 0.28 & 0.23 & 0.20 & 0.17 \\
\hline & 8 & 0.80 & 0.59 & 0.44 & 0.35 & 0.29 & 0.25 & 0.22 \\
\hline & 10 & 1.07 & 0.71 & 0.54 & 0.43 & 0.36 & 0.31 & 0.27 \\
\hline & 12 & 1.27 & 0.85 & 0.63 & 0.51 & 0.42 & 0.36 & 0.32 \\
\hline & 14 & 1.47 & 0.98 & 0.73 & 0.59 & 0.49 & 0.42 & 0.37 \\
\hline
\end{tabular}
$D_{R}$ the RMS-error in $D_{R}$, denoted by $\left(D_{R}\right)$, can be expressed as:

$$
\sigma\left(D_{R}\right)=\left(10^{4} \sigma_{M}^{2}+\sigma_{D}^{2} D_{R}^{2}\right)^{\frac{1}{2}} / D_{b}
$$

Table 5.2: Theoretical RMS-error in the detected relative change in vessel diameter as function of the vessel diameter measured along the ultrasound beam $\left(D_{b}\right)$ and true relative change in diameter $\left(\Delta D_{b}\right)$.

Table 5.2 summarizes the RMS-error in $D_{R^{\prime}}$ ' expressed in percents, as function of $D_{b}$ and $\Delta D_{b}$. It can be concluded from this table that for $D_{b}=10 \mathrm{~mm}$ and $\Delta D_{b}=10 \%$ the RMS-error $\sigma\left(D_{R}\right)=0.43 \%$. The position of the vessel wall can only be estimated within a range of $l \mathrm{~mm}$ if the vessel wall induces a distinct low fre- 
quency Doppler signal as function of depth. Clearly, this condition is not satisfied for rough surfaces and the error in the estimated vessel diameter will increase accordingly. But even for $\sigma_{D}=0.82 \mathrm{~mm}$ the RMS-error $\sigma\left(D_{R}\right)$ will be less than 18 for $\mathrm{D}_{\mathrm{b}}=10 \mathrm{~mm}$ and $\Delta \mathrm{D}_{\mathrm{b}}=10 \%$. In the system the relative change in $d i-$ ameter is computed in a digital way and expressed by a 7 bit number with one bit behind the binary point (i.e. expressed as multiples of $0.5 \%$ ). This is in accordance with the anticipated RMS-error in $D_{R}$.

\subsection{ULTRASONOGRAPHIC BLOOD FLOW IMAGING}

Two-dimensional B-mode pulsed echo systems enable transcutaneous real-time visualization of tissue interfaces like vessel walls and can be used to detect irregularities in the vessel wall structure. However, non-calcified plaques do not return signals of sufficient amplitude to be sensed by the echo system. Therefore, the system will fail to show these abnormalities although the plaques cause local narrowing of the blood stream. To show this narrowing a Doppler system can be used (Mozersky, 1971; Haskway, 1980). The ultrasound probe is placed in a position sensing scanning arm. The position information is applied to the $x$ - and $y$-input of a memory display while the $z$-input is activated by the recorded velocity information. In the most simple case the display is only activated if the velocity exceeds a pre-selected threshold. Manual scanning of a surface area will result in a projection of the arterial tree in that area. This approach is widely used in CW-Doppler systems (Lewis, 1978; Berry, 1980) but also finds its application in (multi-gate) pulsed Doppler systems (Fish, 1973; Fish, 1978; Day, 1976; Bournat, 1977; Lusby, 1981).

The possibility of velocity imaging is also incorporated in this system. Unlike a CW-system a pulsed Doppler system has three degrees of freedom: 2 coordinates at the surface and one depth coordinate. Since only 2 degrees of freedom are available 
on a display, a selection of the projection plane, i.e. the combination of 2 coordinates has to be made. This implies 3 options for the projection plane: lateral, cross-sectional and longitudinal view. With a front-panel switch the projection view can be selected while the z-input of the memory display is provided by a comparator, comparing the velocity signal with a threshold under front-panel control. The threshold with respect to the recorded velocity distribution along the ultrasound beam can be visualized on the CRT for the instantaneous velocityprofile. One may select a positive and/or a negative threshold depending on the application (direction of the blood stream with respect to the ultrasound transducer). Two of the processing cycles which are not yet used for the display of other sources of information (velocity distribution, amplitude of echo, vessel wall, and Doppler) are utilized for the display of these thresholds. Blood flow imaging with multi-gate pulsed Doppler systems requires some skill of the investigator because axial movements of the transducer may easily result in motion artefacts causing a detection of blood flow exceeding the threshold at places where it is not present. These motion artefacts are induced by tissue interfaces causing higher Doppler frequencies than anticipated due to the axial movements of the transducer. The first high-pass Doppler filter with the ADC gets then saturated and an unreliable signal is used for further processing.

\subsection{SYSTEM DESCRIPTION}

In the previous sections the basic features of the developed multi-gate pulsed Doppler system with serial data processing has been discussed in detail. Table 5.3 summarizes the characteristics of the system. Since the duration of emission can be set at either $0.5 \mu$ or 1 lus the signal-to-noise ratio can be enhanced by selecting the longest emission duration at the expense of resolution (the dimension of the sample volume in the axial direction will increase). The selection of PRF=19 
Emission frequency

Pulse repetition frequency

Duration of emission

Gate-width

Number of gates

Accuracy detection vessel wall movements
$6 \mathrm{MHz}$

19 or $9.5 \mathrm{kHz}$

0.5 or 1 us

$2 / 3$ HS

$128(8)$

$13 \mu \mathrm{m}$

Table 5.3: Basic technical characteristics multi-gate pulsed Doppler system.

$\mathrm{kHz}$ will limit the maximal depth of investigation to approximately $36 \mathrm{~mm}$. To assess velocities at a greater range a PRF of $9.5 \mathrm{kHz}$ should be selected, doubling the maximal range of investigation. However, this range can not be fully utilized because the signal-to-noise ratio will deteriorate with depth of exploration. In practical conditions the useful range is limited to 40-50 mm. The emitted acoustical power for a PRF of $9.5 \mathrm{kHz}$ and a duration of emission of $0.5 \mathrm{\mu s}$ is equal to $14 \mathrm{~mW}$.

The interval at which phase samples can be selected is either $0.5 \mathrm{~mm}$ or $1 \mathrm{~mm}$. Because of the large number of sample volumes that can be selected (maximal 128) a sampling interval of $0.5 \mathrm{~mm}$ in combination with a duration of emission of $0.5 \mathrm{\mu s}$ should be preferred to preserve detail in the assessed velocity distribution along the ultrasound beam.

The emitter/receiver section is depicted in fig. 5.15. A wideband emitter is employed to energize the probe. Since the high-voltage emitted burst will cause saturation and even damage the input amplifier, the receiver section input is protected by a limiter, restricting the signal input amplitude to 0.6 volt. Despite this protection saturation can still occur, requiring some recovery time for the input amplifier (and demodulator). That is why sampling is only activated after 2 is with respect to the time of emission. 


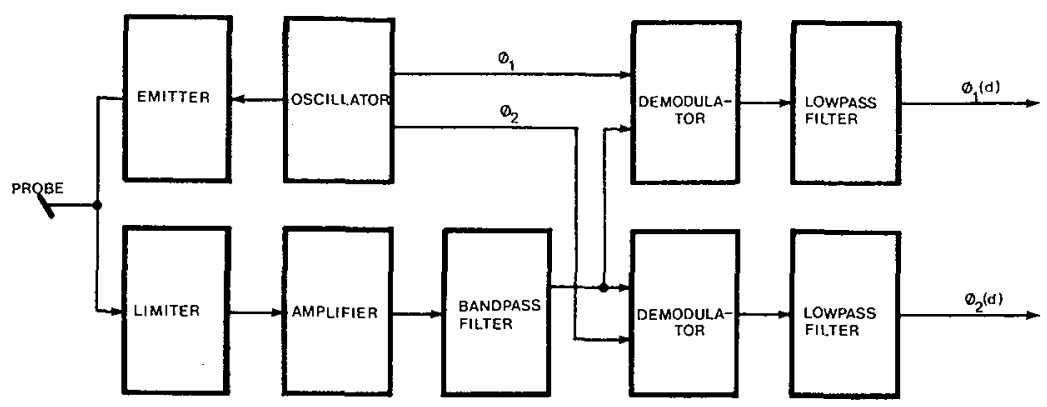

Fig. 5.15: Diagram of the pre-processing circuit. The oscitlator provides two in quadrature reference signals $\left(\phi_{1}\right.$ and $\phi_{2}$ ) for the phase detector resulting in two in quadrature phase signals as a continuous function of depth.

The band-pass filter limits the signal bandwidth to improve the signal-to-noise ratio. On the other hand (see section 4.5) a specific bandwidth is required to maintain the axial resolution. Band-pass filtering is achieved by a double tuned LCcircuit. The $3 \mathrm{~dB}$ bandwidth is set at $1.2 \mathrm{MHz}$, suitable to accommodate bursts with a duration of $0.5 \mu \mathrm{s}$. The output of the band-pass filter is fed into the in quadrature demodulator. The in quadrature reference signals $\left(\phi_{1}\right.$ and $\left.\phi_{2}\right)$ are provided by the oscillator. A low-pass filter of the third order with $3 \mathrm{~dB}$ cutoff frequency of $650 \mathrm{kHz}$ removes spurious frequencies produced by the demodulator and provides a running average of the phaseoutput.

The overall performance of emitter, transducer, receiver and phase-detector section can be evaluated by aiming the transducer at a small target (human hair, thickness $40 \mu \mathrm{m}$ ). The envelope as function of range of the in quadrature demodulated signals provides the necessary information about the axial performance of the system. The envelope can be readily obtained since the system is capable to assess the amplitude of the received signal as function of range (see section 5.6). Manipulating the range at which the target is located by axial translation of the probe in steps of $50 \mu \mathrm{m}$ and measuring the echo amplitude at a given range with respect to the transducer, results in the 
74

axial shape of the sample volume as depicted in fig. 4.2 (the amplitude values are on an arbitrary scale). Taking the -6 dB level with respect to the peak amplitude as boundaries of the sample volume in the axial direction, it can be concluded that the overall axial resolution of the system is less than $0.8 \mathrm{~mm}$. This is worse than the anticipated $0.5 \mathrm{~mm}$ but one should keep in mind that this figure represents the overall performance including the transducer. If only the input/demodulator section is considered (injecting an electric signal) the result would be more favourable.

The lateral dimension of the sample volume is determined by the local width of the ultrasound beam. The employed transducer (diameter $5 \mathrm{~mm}$ ) is focussed at a depth of $20 \mathrm{~mm}$ using a concave

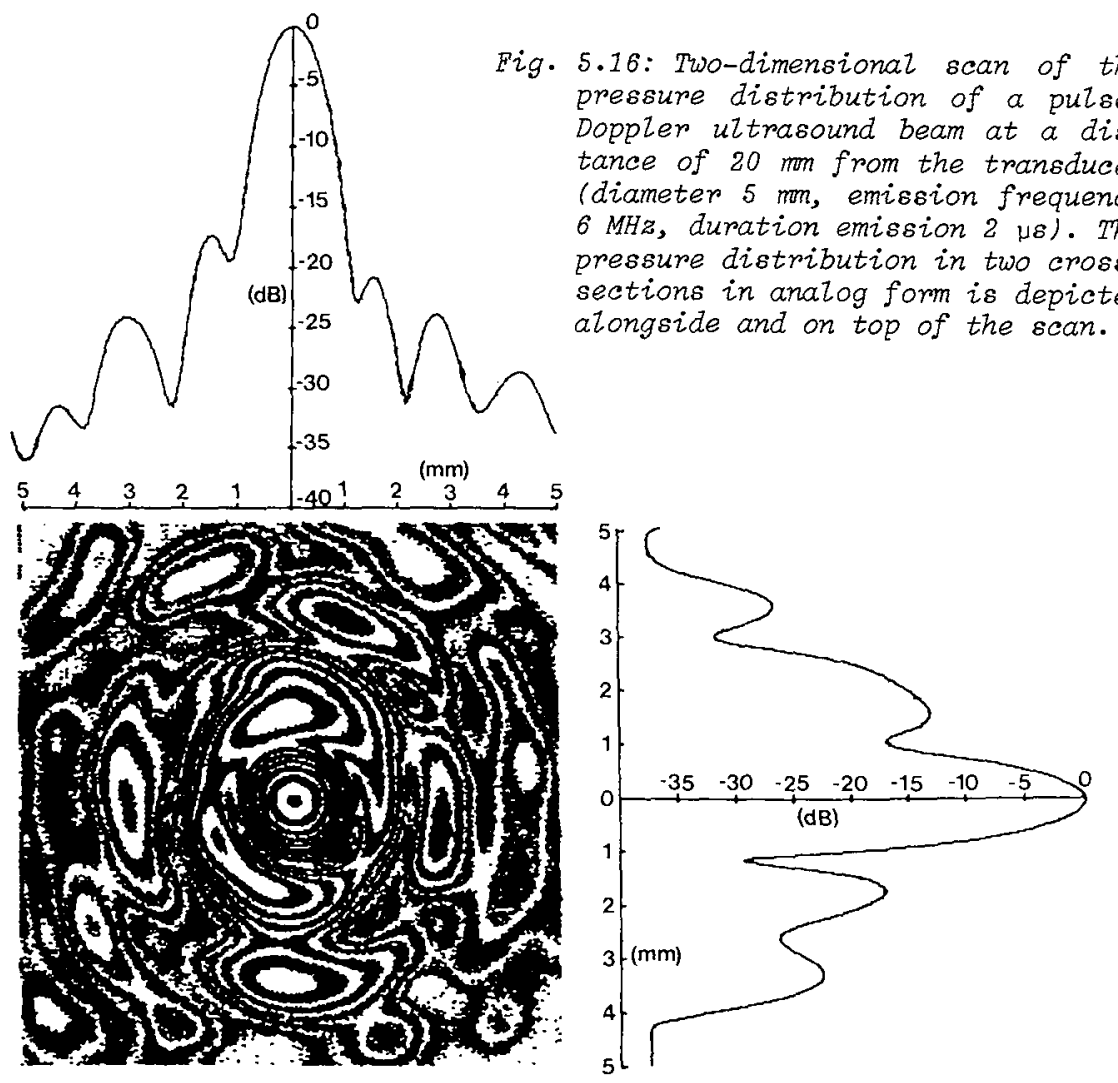


perspex lens. The beam plot as measured at a depth of $20 \mathrm{~mm}$ is given in fig. 5.16. The ultrasound beam is rather axisymmetric as is confirmed by the analog tracings alongside and on top of the diagram, representing the pressure distribution in two cross-sections passing through the center of the ultrasound beam. We will assume that the transducer characteristics for emission and reception are the same. The cumulative received signal intensity as function of the distance to the center of the beam is depicted in fig. 5.17. From this figure it can be

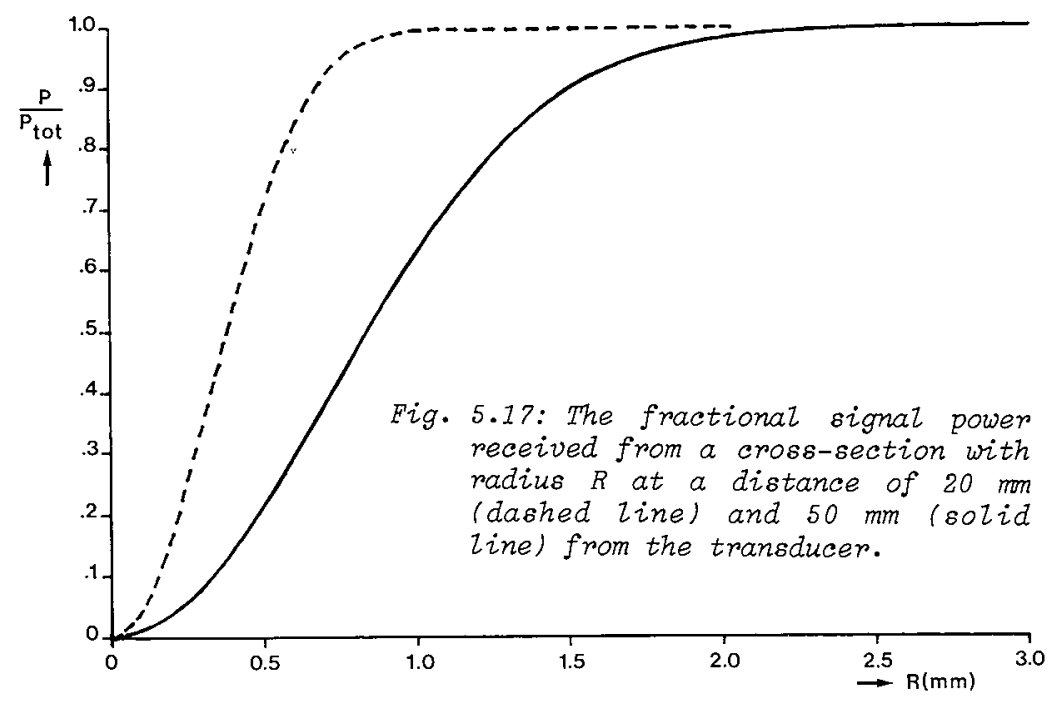

concluded that at a range of $20 \mathrm{~mm}$ (dashed line) a crosssection with a diameter of $1 \mathrm{~mm}$ contributes about $68 \%$ to the received signal energy while at a range of $50 \mathrm{~mm}$ (solid line) the same percentage originates from a cross-section with a diameter of $2.2 \mathrm{~mm}$. The increase in diameter at a greater range is due to the divergence of the ultrasound beam. We are now able to express the size of the sample volume based on the results presented in fig. 4.2 (axial performance) and fig. 5.16 (lateral performance). In fig. 5.18 the fractional received power is depicted as function of the size of the sample volume located at a depth of $20 \mathrm{~mm}$. It can be concluded that a volume $1 \mathrm{~mm}^{3}$ contributes $78 \%$ to the received signal power. 


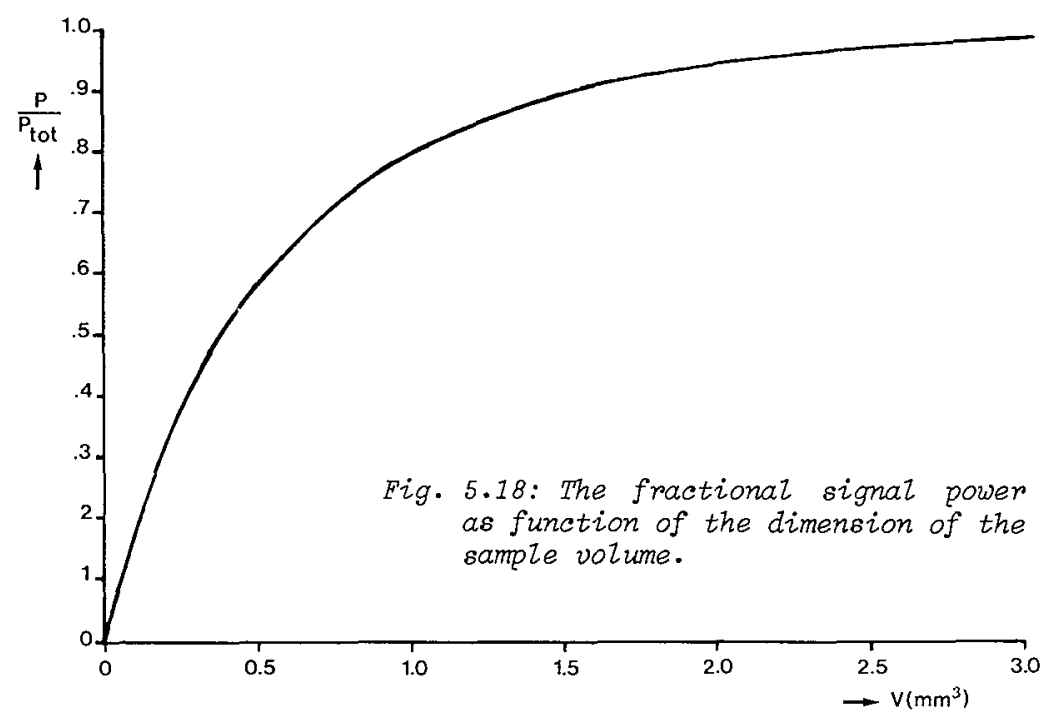

A concise block-diagram of the discussed pulsed Doppler system is depicted in fig. 5.19. A detailed description of the preprocessor was already given in fig. 5.15. The organization of the hybrid filter of the first order (including the analog to digital converter) was discussed in section 5.4. For detection of the relative changes in vessel diameter (relative

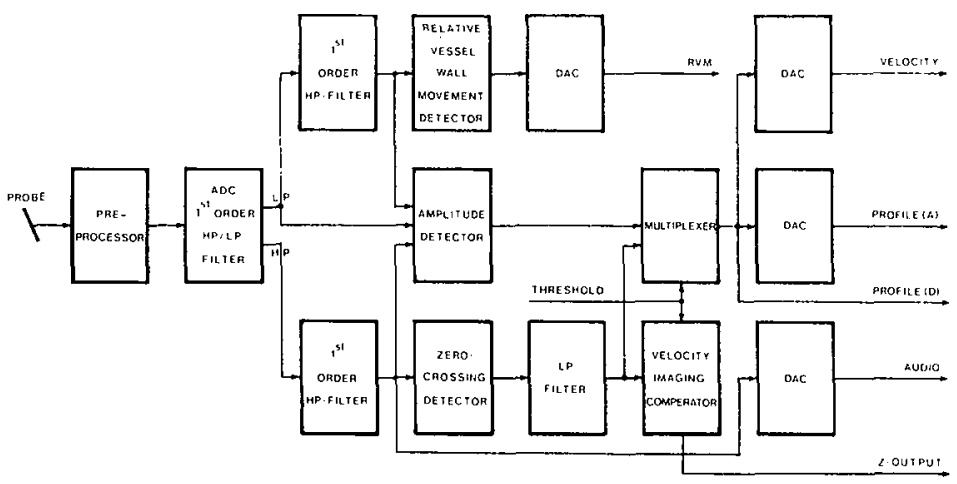

Fig. 5.19: Concise block-diagram of the multi-gate pulsed Doppler system with serial data-processing. The detected velocity-distribution along the ultrasound beam is available in both analog (A) and digital form (D). The pre-processor is depicted in fig. 5.15 . 
vessel wall movements) the low-pass output is high-pass filtered (see section 5.7). Employing a digital to analog converter the relative vessel wall movements (RVM) are obtained in analog form. The high-pass output of the hybrid filter is fed into a second high-pass filter of the first order to increase the roll-off. The filtered value of a selected sample volume is fed into a DAC for audio-evaluation of the assessed Doppler-signal. To obtain the velocity distribution along the ultrasound beam, the in quadrature Doppler signals are fed into a zero crossing detector followed by a digital low-pass filter (section 5.5). The detected velocity profile is available in both analog (A) and digital (D) form. For arterial velocity imaging (section 5.8) the velocity-signals of all the activated sample volumes are compared with a threshold. For those gates where the velocity exceeds the threshold the z-input of a memory CRT is activated. To detect the envelope of the assessed Doppler signals in the various frequency ranges the low-pass output of the hybrid filter, the high-pass output of the filter for vessel wall movements or the high-pass output of the Doppler filter is fed into the amplitude detector. To obtain a composite display of all the basic information (signal envelope, velocity, distribution, and threshold) on a single CRT a multiplexer followed by a DAC is employed. The display rate is fixed at 1200 $\mathrm{Hz}$.

\subsection{REAL-TIME DISPLAY OF TIME-DEPENDENT VELOCITY PROFILES}

On the monitor the instantaneous velocity distribution sensed along the ultrasound beam is displayed at a rate of $1200 \mathrm{~Hz}$. The presented velocity profile enables the selection of the sample volume for audio evaluation based on visusal inspection of the velocity distribution. It provides also information about the symmetry and the width of the profile and the peak velocities occurring in a cardiac cycle. However, this realtime display is unsuitable for the evaluation of the profile as it develops in a cardiac cycle since this requires the simul- 
taneous display of previous assessed velocity profiles, temporarily stored in a memory. A dedicated memory unit is incorporated in the multi-gate pulsed Doppler system for the on-line evaluation of the time-dependent behaviour of the velocity distribution.

A basic decision that has to be made is the way of representing the three degrees of freedom (position, time, velocity) on the screen. The decision is complicated by the fact that the velocity may appear with both polarities (positive and negative flow velocity). Associating the $x$ and $y$ coordinates of the display with time and position and representing the velocity in shades of grey will therefore lead to misinterpretation of the polarity of the velocity. That is why some investigators opted for a color coded display of velocities to enable easy evaluation of the assessed velocities with respect to each other (Brandestini, 1979; Eyer, 1981; Stevenson,1981). However, the time-dependent behaviour of the velocity in a given sample volume is obscured by the quantity of information displayed simultaneously. In a color coded display it will be problematic to extract some basic parameters as velocity, expressed in $\mathrm{Hz}$ as instantaneous function of time, the duration of the systolic peak or the width of the velocity profile. To maintain this kind of information we opted for a display format where the velocity is represented as a deflection of a known zero-level and the information of interest can be enhanced by selecting the proper display mode (Hoeks, 1979; Hoeks, 1981; Reneman, 1981a). Two display modes can be distinguished, the velocity as a continuous function of depth at aiscrete time intervals and the velocities in a number of adjacent sample volumes as a continuous function of time. The first display mode emphasizes the spatial behaviour while the second one stresses the temporal characteristics of the sensed velocities. Both display modes utilize the same set of data only the representation differs. At a fixed input rate of either 30 or $60 \mathrm{~Hz}$ the instantaneous velocities in 64 adjacent sample volumes are stored in a 16 kbyte memory organized as 256 (time) by 64 (depth) 
words of 8 bits. Since the multi-gate system is able to assess simultaneously velocities in a maximum of 128 sample volumes a selection can be made of the number of sample volumes to be skipped for the input of the memory. Since the Doppler system and the memory control run independently, data transfer between both systems is achieved via a 128 by 8 bits first in/first out (FIFO) memory. The input clock of the FIFO is provided for by the Doppler system while the output clock is under control of the memory unit. The transfer rate for each of the sample volumes can be selected at either 30 or $60 \mathrm{~Hz}$. The lowest transfer rate allows for a longer time span (more than 8 seconds) over which the velocity can be retained while the higher update rate maintains more detail in the temporal behaviour of the velocities. The update rate of $60 \mathrm{~Hz}$ is well beyond the maximum frequency content of the velocity signal that may be anticipated in peripheral vessels.

The velocity information is displayed in scroll mode i.e. the time axis is maintained without discontinuities while the velocities over the past maximal 8 seconds are represented. Freezing of the information by interrupting the update procedure can be executed at any time. Since for the recording of the relative change of the vessel diameter during the cardiac cycle an ECG trigger is available this trigger can also be utilized to freeze the data synchronously. This offers the advantage that the last cardiac cycle is completely displayed. In the absence of the ECG-trigger, freeze-mode is initiated at the moment the freeze command is issued. The display of data in scroll mode requires that for each new update cycle the write address for the velocity originating from the selected 64 adjacent sample volumes is lowered by one (modulo 256) while read-out has to start at this address. Increasing the memory address (modulo 256) enables the retrieval of velocity information stored previously.

The way the memory is addressed for read-out depends on the selected display mode. The lower 8 address bits are reserved 
for the time coordinate while the upper 6 memory address bits are associated with the depth coordinate i.e. with the 64 sample volumes. To display the velocity as a continuous function of time at a given depths the time coordinate is changed while the depth coordinate is altered at the completion of a tracing. For the representation of the velocity profile at given time points the addressing procedure is just the opposite.

The approach in velocity representation as introduced above will lead to the display of either 64 velocity tracings or 256 velocity profiles. Without zoomming facilities the velocity information will still be obscured by the quantity of information. That is why under cursor control the first and the number of sample volumes of which the velocity should be displayed over a selected time span (also under cursor control) can be adjusted without affecting the update procedure. Simultaneously the display format is adjusted so that the screen size is fully utilized. This requires scaling of the $x$ and $y$ coordinates and the data according to the selected display format. To provide for data calibration the scaling factor for the data is selected so that the distance between the zero level of two adjacent tracings is equivalent to a Doppler frequency of $2 \mathrm{kHz}$ for the velocity tracings and $1 \mathrm{kHz}$ for the profiles. A $2 \mathrm{kHz}$ calibration distance in the time mode is taken to avoid confusion about the zero level in case of a high diastolic velocity. In the profile mode this confusion will not occur since outside the observed vessel the assessed velocity will always tend to zero. To evaluate the velocities displayed in profile mode over a few cardiac cycles either 1 of 2 , 1 of 4 or 1 of 8 (under front panel control) of the stored velocity profiles are displayed giving an additional reduction in the number of profiles to be displayed.

Because of the scaling the display of each data point involves three multiplications ( $x$ and $y$ coordinates plus data). To achieve a high data output rate scaling is executed by a 12 by 12 bit parallel multiplier with a multiplicaton time of only 
120 nsec. Allowing some time for address control and memory access an output rate of $1 \mathrm{MHz}$ can thus be achieved. The display of, for example, a 128 (time) by 36 (sample volumes) display frame will require approximately $8 \mathrm{~ms}$ allowing a frame rate in the order of $120 \mathrm{~Hz}$. The frame rate, however, is reduced by a factor of 2 because of interpolation between 2 adjacent points to obtain a smooth recording. In these conditions the update procedure will not interfere with the display of data even if the highest update rate is utilized. Larger display frames may only be selected if the update rate is lower or in the freeze mode. To get information about the zero reference level in both aisplay modes the base lines of each tracing are displayed in every 4 display frames. They appear therefore on the screen with a lower intensity than the velocity tracings.

The bottom part of the display has been reserved for the display of the relative change in vessel diameter as function of time. For this tracing data scaling is independent of the selected display format, only the time axis is scaled accordingly. The occurrence of an ECG-trigger is represented by a negative deflection which for calibration purpose is made equivalent to a relative change in diameter of $10 \%$.

For filing purposes the displayed data can be transferred to an $x-y$ plotter. The time required to complete a plot depends on the format and will be in the order of 1 to 5 minutes.

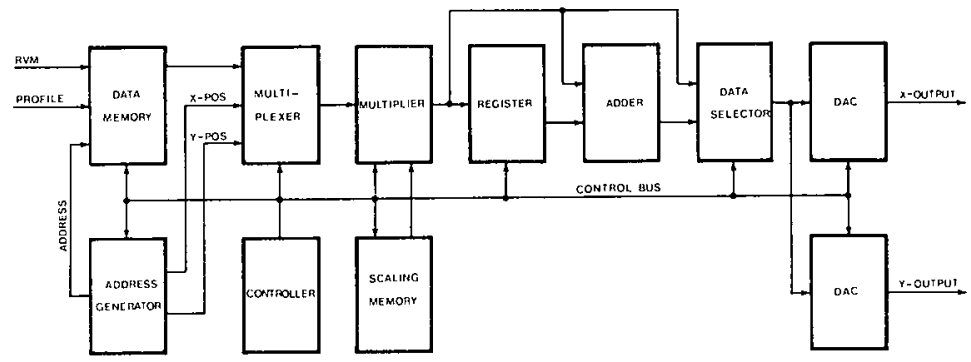

Fig. 5.20: Block-diagram for the display of either the observed velocity distribution as function of time or the velocity signals in a number of sample volumes as function of time. 
82

In fig. 5.20 the basic configuration of the memory unit is depicted. The address generator provides both the read/write address of the memory and the coordinates for the screen. The multiplexer at the multiplier input selects whether the $x$-coordinate, $y$-coordinate or data has to be scaled. The scaling factors are provided by a ROM which is addressed according to the selected display format. The register at the output is

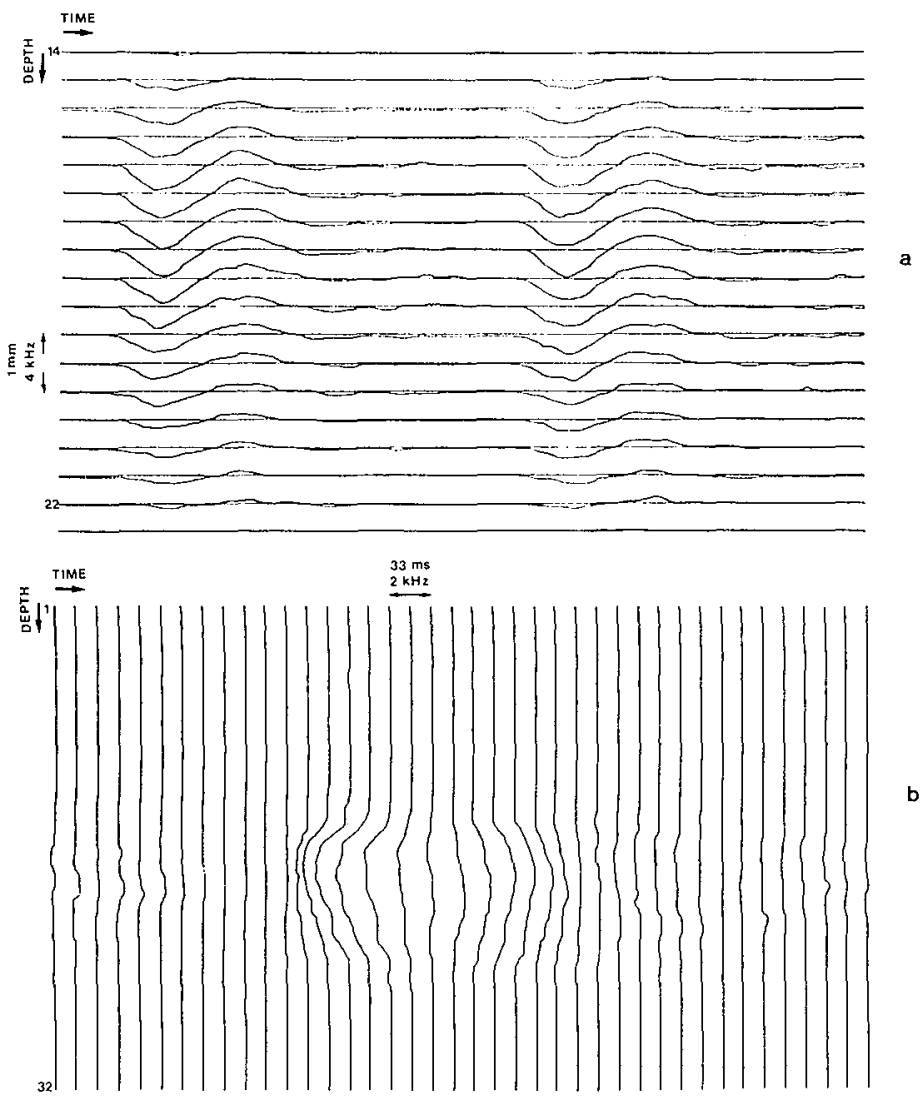

Fig. 5.21: The velocity in a number of adjacent sample volumes spaced at $0.5 \mathrm{~mm}$ intervals as a continuous function of time, recorded from the superficial femoral artery. The distance between the zero-level of two adjacent tracings is equivalent to a Doppler frequency of $2 \mathrm{KHz}$. Fig. 5.21 b shows the recorded velocity-distribution along the ultrasound beam at discrete time-intervals 133 ms) based on the last cardiac cycle shown in fig. 5.21a. Calibration-level $1 \mathrm{KHz}$. 
utilized for the intermediate storage of the off-set for each of the tracings. To which DAC the scaled data has to be transferred depends on the selected display mode. All the blocks are under control of a dedicated controller operating at $6 \mathrm{MHz}$. Fig. 5.21 shows an example of the output of the memory unit in both output modes. The profile mode is based on the same data as the velocity mode but, because of the way data is represented, different characteristics are emphasized.

\subsection{DISCUSSION}

In the system as described in the previous sections the processing of Doppler signals (filtering, detection of average Doppler frequency and signal envelope), originating from the activated sample volumes, is executed in serial form, ensuring that processing is identical for all signals. Therefore, the characteristics of the processing circuitry as discussed for a single sample volume automatically applies for all involved sample volumes. The required hardware is virtually independent of the selected number of sample volumes. However, the maximum number of sample volumes is limited by the maximum range of exploration set by the PRF. A high PRF is necessary to be able to assess reliably velocities under physiological and pathophysiological conditions.

Small sample volumes (in the order of $1 \mathrm{~mm}^{3}$ ) allow the assessment of a detailed velocity distribution along the crosssection of major peripheral arteries (e.g. carotid and femoral arteries). Local disturbances in the velocity pattern induced by abnormalities of the vessel wall can be detected by the presented system. This is illustrated by an in vitro experiment where an axisymmetric stenosis was simulated in an elastic tube with a diameter of $14 \mathrm{~mm}$. The velocity pattern was observed 1 $\mathrm{cm}$ from the constriction under an angle of $60^{\circ}$ with respect to the axis of the tube. The volume flow through the tube was sinusoidal (no DC component) with a frequency of $1 \mathrm{~Hz}$. Because 
the volume flow will change direction two times per second the velocity pattern is observed alternately distal and proximal to the constriction (both for 508 of the time). Fig. 5.22a shows the recorded velocity pattern for a constriction of 08 , pre-

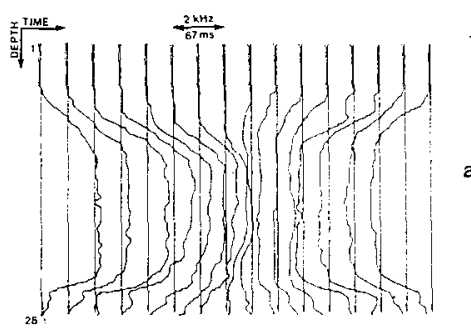

Fig. 5.22: In vitro recordings of velocity distribution along ultrasound beam. Diameter of elastic tube 14 m. Angle of observation $60^{\circ}$. Registrsations are made $1 \mathrm{~cm}$ from a local contriction of $0 \%$ (no wall movements) $8.3 \%$ in diameter (fig. $5.22 a$ and $\mathrm{fig} .5 .22 b$ ) respectively. Velocity averaged over cross-section is sinusoidal with a frequency of $1 \mathrm{~Hz}$. Since the flow reverses direction twice a second part of

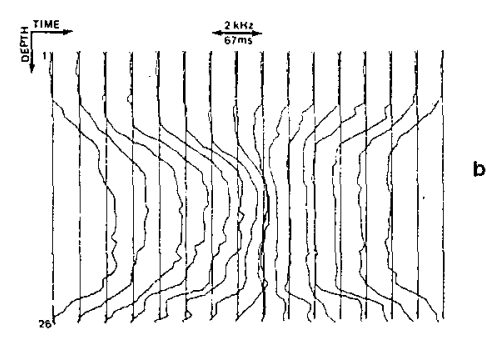
the registration is made distal to (Left) and part of it is made proximal (right) to the artificial stenosis. Even a constriction of $0 \%$ (fig. 5.22a) has considerable effect on the velocity distribution distal to the stenosis. A narrowing of the profile in combination with higher centrum stream velocities can be observed in fig. $5.22 b$.

venting thus movements of the wall of the tube (otherwise the relative change in diameter would be $10 \%$ ). The velocity distribution distal to the constriction (left part) is distinctly different from the recordings made proximal to the constriction (right part). In the left part the peak velocities are higher while the velocities recorded near the wall are lower. Fig. $5.22 \mathrm{~b}$ shows the velocity tracings under the same recording conditions except for a local reduction in diameter of $8.3 \%$. In the recordings made distal to the constriction (left part) the peak velocities near the axis of the tube are higher as compared to those shown in fig. 5.22a. Moreover, a narrowing of the profile can be observed, especially during the last $200 \mathrm{~ms}$ before flow reversal takes place. The recordings made proximal to the constriction (right part) do not differ significantly from those shown in fig. $5.22 \mathrm{a}$.

Although the system is capable to assess in detail the velocity distribution along the cross-section of the artery it is 
not able to detect volume flow. To estimate the volume flow one needs simultaneous information about the cross-section of the and the velocity averaged over the cross-section of the investigated artery (Brandestini, 1976). Since, no a priori information is availabe about the angle of observation the true diameter of the artery cannot be calculated straightforward from the diameter as observed along the ultrasound beam (see section 5.7). Even more complicated is the detection of the velocity averaged over the cross-section of the artery. Basic requirements are the intersection of the axis of the ultrasound beam and the axis of the vessel throughout the cardiac cycle and rotation symmetry of the profile. Under these conditions the average velocity can be calculated by integrating the velocity pattern where the contribution of the sample gate depends on its location with respect to the axis of the vessel (Roevros, 1974). The weighing function is necessary to correct for the partial observation of the off-axis velocities and depends on the diameter of the ultrasound beam with respect to the diameter of the artery. The center of the weighing function should coincide with the axis of the artery throughout the cardiac cycle. The complexity of the operations necessary to obtain the velocity averaged over the cross-section of the artery impedes an on-line approach. A simpler method to assess the average velocity would be the employment of a pulsed Doppler system where the axial and lateral dimensions of the sample volume are slightly greater than the diameter of the artery in combination with an echosystem. The combination of both systems provides information about the angle of observation and the diameter of the artery. To avoid interaction between both systems the echo and Doppler system should be activated alternatedly for a period long enough to assess either the diameter or the average velocity (in the order of $10 \mathrm{~ms}$ ). 



\section{IMPROVEMENT OF THE FREQUENCY DETECTOR CHARACTERISTICS}

\subsection{INTRODUCTION}

The operation of multi-gate pulsed Doppler systems heavily depends on the assessed velocity distribution rather than the audio-evaluation of the Doppler signals originating from a selected sample volume. It is therefore essential that the error in the detected average velocities remains small independent of recording conditions as signal-to-noise ratio, Doppler bandwidth and average Doppler frequency in relation to the PRF.

A widely recognized source of error in pulsed Doppler systems is frequency ambiguity occurring if the induced Doppler frequencies exceed the Nyquist frequency i.e. PRF/2. To circumvent this basic problem investigators (Newhouse, 1980; Cathignol, 1980) attempted to increase the PRF without introducing range ambiguity (see section 4.1). In principle this can be achieved by coding the emitted ultrasound bursts. The received signals are then analyzed to reveal to which emission burst they are related to. The unambiguous Doppler frequency range will be extended by a factor of 2 if an alternating code for the emitted signals is employed. This approach requires some additional pre-processing and can be implemented in single-gate pulsed Doppler systems. For multi-gate pulsed systems with serial data processing it is less attractive because of the necessary data manipulation requiring complicated digital circuits.

Another method to resolve frequency ambiguity without the associated increase in PRF is based on the evaluation of the time-dependent behaviour of the spectral composition of the detected Doppler signals. The distribution of the reciprocal values of the time-intervals elapsing between two successive zero crossings as obtained with a directional zero crossing detector (time interval histogram) provides a simple mean to 
88

get some insight in the spectral composition (Burckhardt, 1981). The display of the time interval histogram reveals the incorrectly detected zero crossings due to frequency aliasing. Based on the distribution of the time-intervals and its timeaverage a correction algorithm can be employed to correct for frequency aliasing (Hartley, 1981). Obviously, this procedure will extend the velocity range of the pulsed Doppler system, although the audio output does not reflect the spectral composition of the Doppler signal.

The above method can be employed in pulsed Doppler systems (single gate or multi-gate with parallel data processing) where the assessed Doppler signals are available as a continuous function of time. However, if the processing is based on the sampled values of the Doppler signals (multi-gate system with serial data processing) other errors are introduced. The timediscrete zero crossing detector (see section 5.5) is unable to resolve properly the direction of the observed velocity if the actual Doppler frequency exceeds PRF/4. The situation becomes more complicated if the Doppler frequency exceeds the Nyquist. frequency causing frequency aliasing (see section 6.2). To what extent the signal-to-noise ratio, the shape of the frequency distribution and the average frequency of the Doppler signal will affect the average frequency as detected with a time-discrete zero crossing detector is difficult to predict analytically. Therefore, we performed some computer studies (see section 6.3) to evaluate experimentally the relation between the observed error in the output of a frequency detector and the main signal characteristics (signal-to-noise ratio, bandwidth and average frequency of the Doppler signal).

The computer experiments confirm (see section 6.4) that for a poor signal-to-noise ratio, especially in combination with a high average Doppler frequency, large errors occur in the average frequency as detected with a time-discrete zero crossing detector. This can be partly attributed to the property of the 
frequency detector to resolve properly the sign of the frequency components of the assessed Doppler signal over a limited $(-\mathrm{PRF} / 4$ to $\mathrm{PRF} / 4)$ frequency range. Direction ambiguity occurs since the signs of the in quadrature Doppler signals are only able to indicate the position of the phase vector within an interval of 90 (quadrant of the phase plane). A more accurate calculation of the position of the phase vector will extend the frequency range for unambiguous detection of the direction, since then the change in position of the phase vector in subsequent processing cycles can be computed more precisely. The change in phase per unit of time is known as the instantaneous frequency (see section 6.5). The spectral distribution of the instantaneous frequency of a given signal (see section 6.6) is affected by the way the instantaneous frequency is obtained. Using the in quadrature sampled values of the Doppler signal to retrieve the instantaneous frequency will cause processing errors quite similar to frequency aliasing. Therefore, a frequency estimator based on the time-average of the time-discrete instantaneous frequency is unreliable. However, a simple algorithm can be employed to correct for processing and frequency aliasing errors (see section 6.6). This processing scheme is based on the assumption that detected instantaneous frequencies deviating more than $\mathrm{PRF} / 2$ from the detected average frequency are due to projecting (mapping) of instantaneous frequencies located originally outside the interval $-\mathrm{PRF} / 2$ to $\mathrm{PRF} / 2$ into the interval $-\mathrm{PRF} / 2$ to $\mathrm{PRF} / 2$. Adding (or subtracting) a proper number of the PRF so that the deviation gets less than $P R F / 2$ corrects for this mapping. The time-average of the corrected instantaneous frequency is used as reference in the correction procedure.

The procedure as outlined above has two distinct advantages. First, the application of the correction algorithm results in a distribution of the corrected instantaneous frequency which is more or less symmetrically around the detected average frequency. As a consequence the contribution of noise to the de- 
90

tected average frequency will vanish (see section 6.7) and, hence, the anticipated error in the output of the Erequency estimator will be minimal. Secondly, the correction algorithm takes automatically care of aliasing errors. Under specific restrictions with respect to the spectral distribution of the Doppler signal the frequency detector will be able to track properly average Dopoler Erequencies beyond the Nyquist frequency (see section 6.8 ).

A frequency detector based on the corrected instantaneous frequency can easily be realized in hardware. Since the involved data manipulation is straightforward (see section 6.9) no problems are encountered to implement the processing scheme in a system with serial data processing.

\subsection{FREQUENCY ALIASING}

If a monochromatic (single frequency) signal is injected electronically into a pulsed Doppler system then the detected frequency shift $f_{D D}$ will linearly follow the frequency shift $f_{D}$ of the injected signal for frequency shifts within the interval -PRF/2 through PRF/2 (fig. 6.1). However, if $\mathrm{f}_{\mathrm{D}}$ l $>\mathrm{PRF} / 2$ then the detected frequency will differ an integral number of the

Fig. 6.1: A one-to-one relationship between the detected Doppler frequency $f_{D D}$ and the induced frequency $f_{D}$ exists only for the interval -0.5 through 0.5 (times the PRF).

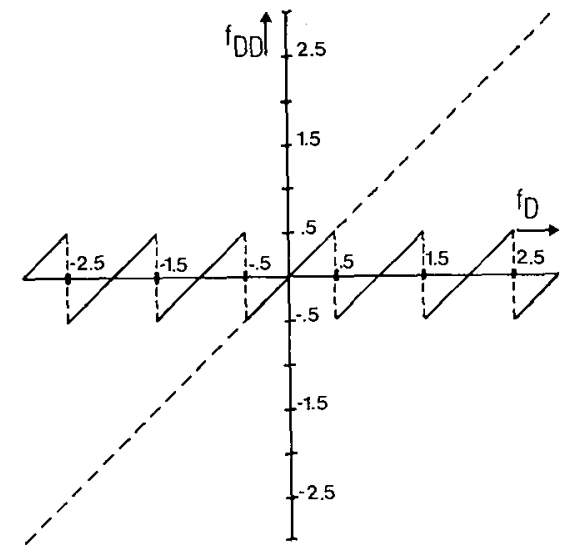


PRF from the true frequency. All the frequencies are mapped into the frequency interval -PRF/2 through PRF/2. The result will be a sawtooth as depicted in fig. 6.l. Only if $\left|f_{D}\right|<P R F / 2$ an unambiguous relation exists between $E_{D}$ and $E_{D D}$. The phenomenon of frequency mapping if the signal is not sampled properly is known as frequency aliasing.

The situation becomes more complicated if a signal is injected with a non-zero bandwidth around an average frequency $\overline{\mathrm{f}}_{\mathrm{D}}$. In fig. 6.2 the allowable frequency distribution (blank area) is depicted as function of $\overline{\mathrm{f}}_{\mathrm{D}}$. Both frequency axas are
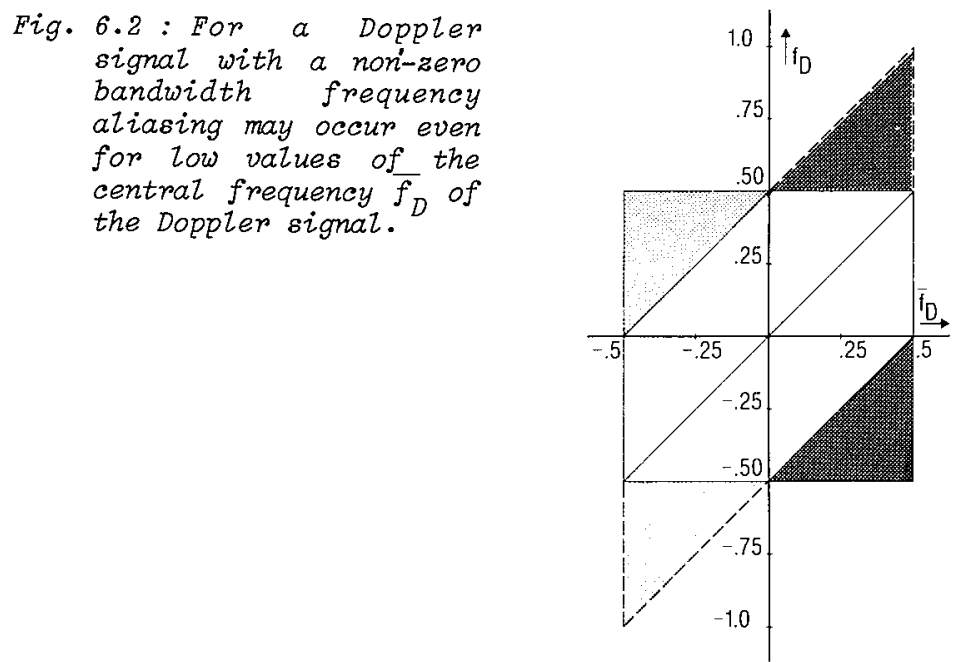

scaled with respect to the PRF. It is assumed that the bandwidth of the injected signal does not exceed the sampling frequency. The shaded areas for $\left|\bar{E}_{D}\right|>0.5$ indicate regions with possible frequency aliasing. Frequencies occurring in this range are mapoed within the Erequency range $\left|\overline{\mathrm{F}}_{\mathrm{D}}\right|<0.5$ (shaded area). Under the assumption that the frequency distribution of the injected Doppler signal is symmetric around $\overline{\mathrm{F}}_{\mathrm{D}}$ the deviation of $f_{D}$ from $\bar{f}_{D}$ that can be tolerated to avoid frequency aliasing is depicted in fig. 6.3. It can be observed that the allowable frequency range narrows for increasing $\mid \overline{\mathrm{E}}_{\mathrm{D}}$ and van- 
92

ishes for $\left|\bar{f}_{D}\right|=0.5$. Since at increasing $\left|\vec{E}_{D}\right|$ the bandwidth of the Doppler signal generally increases due to the transit time effect (see section 4.7) the maximum average Doppler frequency will never reach $\{P R F / 2\}$. This effect will become more and more

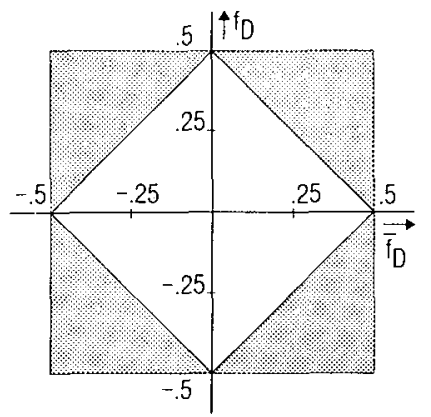

Fig. 6.3 : For increasing $\left|\overline{f_{D}}\right|$ the allowable frequency range (blank area) narrows and vanishes for $\left|\overline{f_{D}}\right|=0.5$. This is in conflict with the observation of increasing signal bandwidth with increasing average frequency.

prominent for a decreasing size of the sample volume. On the other hand increasing the dimension of the sample volume will reduce the spectral widening due to the transit time effect but will introduce spectral broadening induced by the velocity distribution observed within the sample volume.

\subsection{DOPPLER SIGNAL SIMULATION}

The accuracy of a frequency detector depends on several parameters (see section 3.5) like the signal-to-noise ratio, the spectral distribution of the Doppler signal and the spectral distribution of the noise. The effect of these parameters on the performance of various frequency detectors has been evaluated with a computer. Since our main interest is the detection of the average Doppler frequency in a multigate pulsed Doppler system with serial data processing we will only consider those frequency detectors operating on signals available in sampled form. The signal representation and processing in the computer model has been set up accordingly. All the frequencies are expressed with respect to the sampling frequency i.e. the PRF and will appear in the figures non-dimensional. To generate the in quadrature Doppler signals we started with a 
noise signal with a uniform frequency distribution up to $\mathrm{PRF} / 2$ and a Gaussian amplitude distribution (time average equal to zero). Applying a FFT-algorithm the noise signal is converted to the frequency domain (fig. 6.4) where the amplitudes of the negative frequencies are set to zero. Converting the modified frequency spectrum back to the time-domain results in an in quadrature Doppler signal containing only positive frequencies.

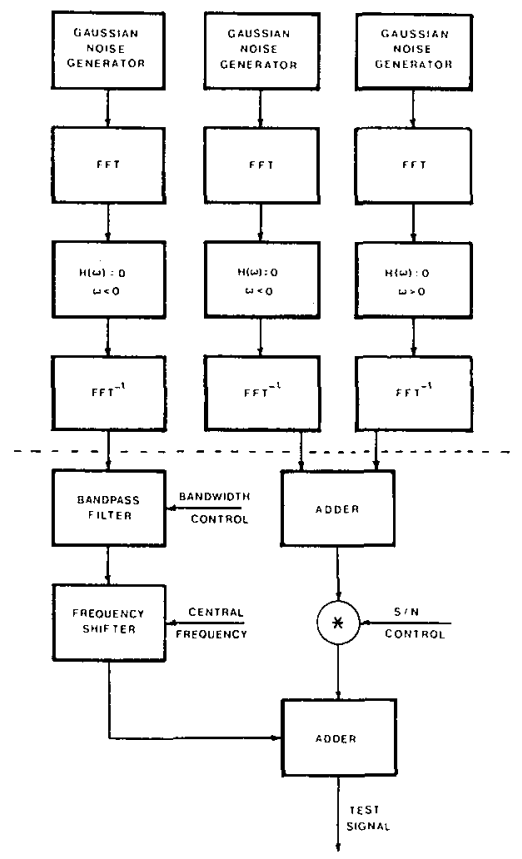

Fig. 6.4 : Flow chart for the synthesis of a Doppler signal. The operations indicated below the broken line are performed in the time-domain. The generated Doppler signal is characterized by its bandwidth, average frequency and the signatto-noise ratio.

Both components of the in quadrature signal are passed through a 10th order bandpass filter with a fixed center frequency (PRF/4) and a pre-settable bandwidth (maximum 0.4 PRF). The filter procedure is executed in the time domain. To generate a signal with a variable average frequency the in quadrature Doppler signal is multiplied by $\exp \left(2 \pi j f_{S} t\right)$ where $f_{S}$ represents the time dependent shift of the average frequency. Setting $f_{S}$ equal to $-\mathrm{PRF} / 4$ results in a Doppler signal with a frequency spectrum distributed symmetrically around zero. In quadrature noise signals with a uniform frequency distribution over the range $-\mathrm{PRF} / 2$ to $\mathrm{PRF} / 2$ are generated by adding two in quadrature 
94

noise signals of which one contains positive frequencies while the other contains negative frequencies. The in quadrature noise signal is added to the in quadrature Doppler signal in a given ratio to obtain a pre-selected signal-to-noise ratio. The generation of the in quadrature signals involves some time consuming Fourier transformations especially since long files are considered ( 8192 or 16.384 points) but for further evaluation the same files can be used. Only the processing in the timedomain (filtering, shifting of frequency, signal-to-noise ratio) has to be repeated for each simulation study. In fig. 6.5 the combined signal and noise spectrum, that can be generated with the aforementioned procedure, is depicted.

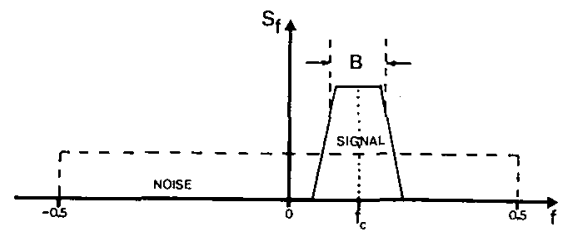

Fig. 6.5: Spectral distribution of the signal and the noise as generated by the computer procedure.

\subsection{EVALUATION OF THE TIME DISCRETE DIRECTIONAL ZERO CROSSING DETECTOR}

In section 5.5 the directional zero crossing detector as employed in the multi-gate pulsed Doppler system with serial data processing was introduced. It has been pointed out that the assignment of the direction was unambiguous if the sampled in quadrature Doppler signals did not change sign coincidently. However, due to the presence of wide band noise and/or high Doppler frequencles with respect to the sampling frequency, direction ambiguity will occur Erequently. In this condition the assigned direction was the direction of the time average of the detected zero crossings. Denoting the in quadrature Doppler signals by $x_{k}$ and $y_{k}$, where $k$ indicates the moment of observation and $y$ is the Hilbert transformed signal of $x$, then the output $\hat{f}_{i}(k)$ of the directional zero crossing detector can be estimated as: 


$$
\begin{aligned}
\hat{\mathrm{E}}_{i}(k)= & \left(-\operatorname{sgn}\left(x_{k}\right)+\operatorname{sgn}\left(x_{k-1}\right)\right) *\left(\operatorname{sgn}\left(y_{k}\right)+\operatorname{sgn}\left(y_{k-1}\right) / 8\right. \\
& +1-\operatorname{sgn}\left(x_{k}\right)+\operatorname{sgn}\left(x_{k-1}\right) ! * \operatorname{sgn}\left(y_{k}\right)-\operatorname{sgn}\left(y_{k-1}\right) ! * \operatorname{sgn}(\langle\hat{f}\rangle) / 8
\end{aligned}
$$

The time-average $\langle\hat{f}\rangle$ of $\hat{f}_{i}(k)$ is expressed as a fraction of the sampling frequency. The first term in expression 6.1 represents the detected zero crossings in $x$ under the condition that $y$ maintained its sign. It can be shown (Angelsen, 1979) that an estimator for the average frequency will have a bias which is related to the bandwidth and the central frequency of the Doppler signal under the condition that the frequency spectrum is limited to PRF/4. Wide band noise will deteriorate further the performance of this frequency estimator. The second term in expression 6.1 contains the zero crossings where $x$ and $y$ changed sign simultaneously. A statistical evaluation of this part of the expression is quite complicated since it involves the solution of a four-dimensional joint probability density function. We therefore prefer to evaluate the combined effect of both terms on the estimator performance under various signal conditions by computer simulation.

Fig. 6.6 demonstrates the effect of Doppler bandwidth and average frequency of the Doppler signal for various signal-tonoise ratios (SNR=30, 20 and $10 \mathrm{~dB}$ respectively) on the estimator output. The relative error is defined as the difference between observed and imposed central Doppler frequency with respect to the imposed central frequency (see expression 3.16). It can be observed that for a given central frequency the error increases with increasing bandwidth of the Doppler signal and decreasing signal-to-noise ratio. Doppler signals with a low central frequency are generally overestimated while at higher central frequencies a substantial underestimation, especially for wide band signals, can be observed. The overestimation for low central frequencies is partly due to the non-linear behaviour of a zero crossing detector (see section 5.5). Moreover, 

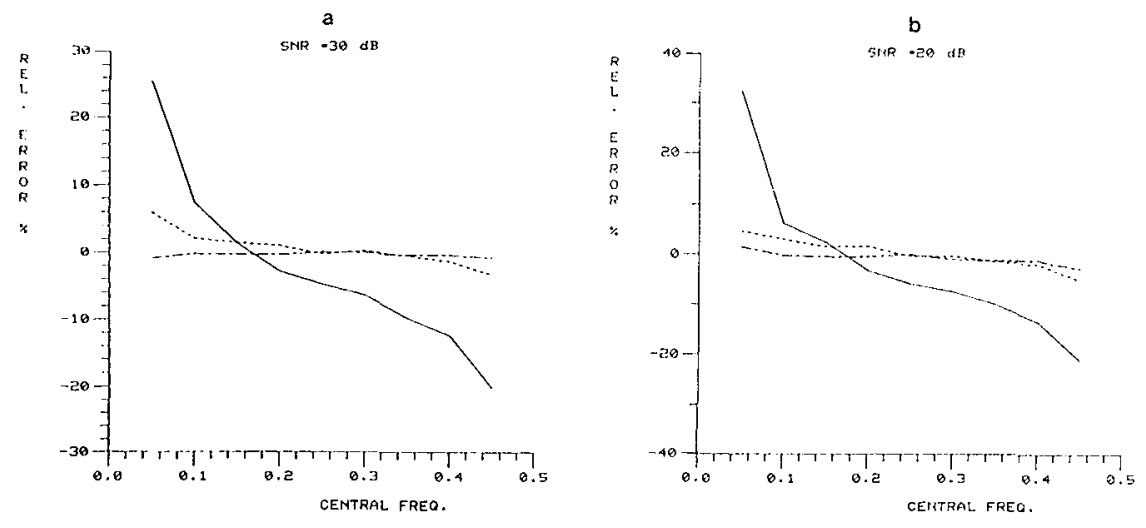

Fig. 6.6 : The observed error in the output of a timediscrete zero crossing detector as function of the average frequency of the simulated Doppler signal for 3 bandwidths $(B=0.01, \quad B=0.1$ and $B=0.3$ ) and for a signalto-noise ratio of $30 d B$ $(6.6 a), 20 \mathrm{~dB}(6.6 \mathrm{~b})$ and $10 \mathrm{~dB}(6.6 \mathrm{c})$. The fractional error is substantial for signals with a large bandwidth (this can be due to a poor SNR).

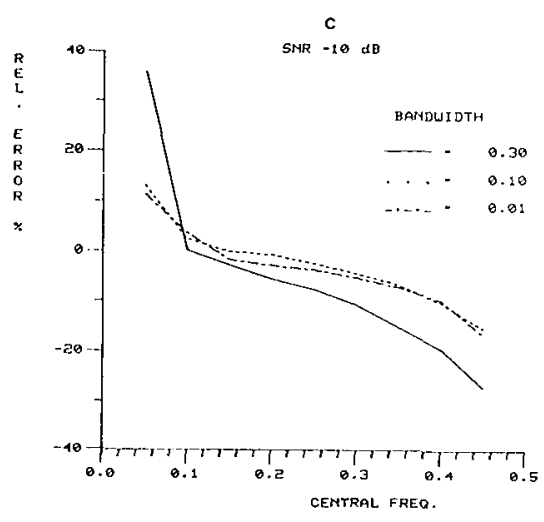

overestimation is caused by the non-linear behaviour of the second term in expression 6.1. Due to noise the signal contains frequencies over the range $-P R F / 2$ to $P R F / 2$. However, all frequencies within the range $-\mathrm{PRF} / 2$ to $-\mathrm{PRF} / 4$ are interpreted as positive frequencies resulting in an overestimation of the central frequency. The underestimation at high central frequencies (PRF/4 to PRF/2) can partly be attributed to the method of assignment of direction, partly to the noise contribution and partly to the way the signal is processed. Although all the information of a signal is contained in its sampled values, provided the sample frequency was properly selected, the sample values are insufficient to detect all the zero crossings. Based 
on the sampled data the minimum detectable interval between successive zero crossings is I/PRF. However, based on the probability density of zero crossings in a band-limited signal (Rice, 1944) it can be concluded that the distance between zero crossings can be much smaller and that, therefore, some zero crossings will be missed in a sampled system causing underestimation at higher central frequencies. This phenomenon should not be confused with frequency aliasing (see section 6.2) since it was assumed that the signal was properly sampled.

In the practical situation frequency aliasing will occur also at higher central frequencies depending on the signal bandwidth. Its effect on the detected average frequency is partly compensated by the way the second term in expression 6.1 takes care for the assignment of the detected velocity direction. However, if the signal frequency exceeds the Nyquist frequency by more than $\mathrm{PRF} / 4$ the detected frequency will fall again in the range $-\mathrm{PRF} / 4$ to $\mathrm{PRF} / 4$ and the first term in expression 6.1 becomes again responsible for the detection of zeroes. The above reasoning applies for both the signal and the noise. For good and fair signal levels (SNR is 30 and $20 \mathrm{~dB}$, respectively) the relative error in the estimator output can be ignored for Doppler signals with a narrow bandwidth (less than 0.1 PRF) but the error becomes substantial for a poor signal to noise ratio $(10 \mathrm{~dB})$.

Because the frequency detector as applied in the multi-gate pulsed Doppler system (see section 5.5) will fail to observe all zero crossings and, moreover, has a non-linear behaviour because of the method of direction assignment it is unsuited to use in a frequency correction scheme as pointed out in section 6.1. An alternative frequency detector, which maintains its direction resolving capability over the full (-PRF/2 to PRF/2) frequency range is based on the detection of the instantaneous frequency (Brandestini, 1978b; Forster, 1978). In the following sections we will pay attention to the basics underlying the 
98

detection of the (time-average of) the instantaneous frequency as applied into a time-discrete multi-gate pulsed Doppler system with serial data processing.

\subsection{THE INSTANTANEOUS FREQUENCY}

The in quadrature Doppler signal can be expressed as:

$$
z(t)=x(t)+j y(t)
$$

where $x(t)$ and $y(t)$ constitute the in quadrature Doppler signal. The instantaneous frequency $f_{i}(t)$ of $z(t)$ is defined as the derivative of the phase:

$$
f_{i}(t)=\frac{1}{2 \pi} \frac{d}{d t} \arctan (y(t) / x(t))
$$

For a time discrete system where the in quadrature Doppler components are only known at discrete time points the above expression changes into (Mandel, 1974):

$$
\hat{\mathrm{f}}_{i}(k)=\left(\arctan \left(y_{k} / x_{k}\right)-\arctan \left(y_{k-1} / x_{k-1}\right)\right) / 2 \pi
$$

where $\hat{f}_{i}(k)$ is expressed as a fraction of PRF. Thus the instantaneous frequency $\hat{\mathrm{f}}_{i}(k)$ is proportional to the rate of change of the phase of the in quadrature signal. Assuming that $z(t)$ contains a single frequency, which is a linear function of time, then the instantaneous frequency will also increase $1 i-$ nearly with time. However, if the signal frequency passes beyond the Nyquist frequency (PRF/2) then the detected instantaneous frequency will change sign abruptly. Hence, we have the same situation as depicted in fig. 6.1. The detected instantaneous frequency will be a periodic function (sawtooth) with a period equal to the $P R F$ and a maximum value equal to $P R F / 2$ (Nyquist frequency). Only if the signal frequency is limited to the interval -PRF/2 through $P R F / 2$ then a one-to-one relation- 
ship exists between the signal frequency and the instantaneous frequency as detected with a sampled system.

However, in the practical situation the Doppler spectrum will have a certain bandwidth due to the shape and size of the sample volume and the velocity distribution within the sample volume. Even if the average velocity (proportional to the average Doppler frequency) remains constant the instantaneous frequency will fluctuate around this average frequency. The probability density function of the instantaneous frequency is related in a complex way to the shape and size of the signal spectrum (section 6.6).

As the signal frequency exceeds the Nyquist frequency the detected value of the instantaneous frequency will differ a factor PRF from the actual value. This can be distinguished if the probability density function of the instantaneous signal frequency is limited to an interval of PRF around its average frequency. If the absolute value of the difference between average and detected instantaneous frequency exceeds $P R F / 2$ it indicates a wrong detection. A simple algorithm exploiting this difference can then be used to correct for the error by adding or subtracting an integral number of PRF such that the corrected instantaneous frequency falls again within the range of PRF around the average frequency. The time average of the corrected instantaneous frequency provides the information to execute the correction algorithm. The computation of the time average with, for instance, a low-pass filter, introduces a phase-lag between the time-average and the actual average frequency. This phaselag may affect the correction procedure more and more as the rate of change in the actual average signal frequency increases. This will be at the expense of the unambiguous range for the instantaneous frequency which becomes smaller at an increasing rate of change.

Introducing the concept of instantaneous frequency and con- 
100

sidering the frequency range of the signal rather than the maximal frequency allows for a less restrictive application of the sampling theorem and in a reduction of the minimal PRF to be used to analyse the sampled signal correctly. Moreover, as a consequence of the above introduced correction algorithm the spectrum of the noise present in the Doppler signal will be altered. Assuming that the noise had originally a flat frequency spectrum, symmetrically around zero, the altered noise spectrum will be distributed symmetrically around the detected average signal frequency. The effect of noise on the detected average frequency will be reduced considerably. This is a very important side-effect of the proposed correction algorithm since pulsed Doppler systems are rather susceptible for noise. It may be expected, therefore, that the error due to noise will be considerable less for a given signal-to-noise ratio if the correction algorithm is implemented.

\subsection{THE PROBABILITY DENSITY FUNCTION OF THE INSTANTANEOUS FREQUENCY}

In section 6.5 the instantaneous frequency was defined as the phase derivative of an in guadrature signal $z(t)$. For a sampled signal the differential equation is replaced by a difference equation. Both expressions will be only equivalent if the time increments are so small that the phase function over this interval can be approximated by a straight line. This condition is evidently not met by the signal $z(t)$ (expression 6.2) sampled by the pulse repetition frequency.

In order to obtain the probability density function $p\left(\hat{\mathrm{f}}_{i}(k)\right)$ for the instantaneous Erequency $\hat{\mathrm{f}}_{i}(k)$ of the sampled signal we recall the expression for $\hat{\mathrm{f}}_{i}(k)$ :

$$
\begin{aligned}
& \hat{\mathrm{E}}_{i}(k)=\left(\omega_{k}-d_{k-1}\right) / 2 \pi \\
& \dot{\phi}_{k}=\arctan \left(y_{k} / x_{k}\right)
\end{aligned}
$$




$$
\phi_{k-1}=\arctan \left(y_{k-1} / x_{k-1}\right)
$$

Under stationary signal conditions the probability for a change in phase of $\phi_{k}{ }^{-\phi} k-1$ is equivalent to $p\left(\phi_{k} ; \phi_{k-1}=0\right)$. Application of Bayes' rule transforms this conditional probability into a ratio of 2 probability density functions:

$$
p\left(\phi_{k} \mid \phi_{k-1}=0\right)=p\left(\phi_{k}, \phi_{k-1}\right) / p\left(\phi_{k-1}\right)
$$

The joint probability density function $\mathrm{p}\left(\phi_{k}, \phi_{k-1}\right)$ is derived by Davenport (Davenport, 1958, section 8.5):

$$
p\left(\phi_{k}, \phi_{k-1}\right)=\frac{D}{4 \pi^{2}} \frac{\left(1-A^{2}\right)^{\frac{1}{2}}+A(\pi-\arccos A)}{\left(1-A^{2}\right)^{3 / 2}}
$$

where $\quad D=11-\rho_{x x}{ }^{2}(T)-\rho_{x y}{ }^{2}(T) !$

$$
\begin{aligned}
& A=\rho_{x x}(T) \cos \left(\phi_{k}-\phi_{k-1}\right)+\rho_{x y}(T) \sin \left(\phi_{k}-\phi_{k-1}\right) \\
& \rho_{x x}(T)=R_{x x}(T) / R_{x x}(0) \\
& \rho_{x y}(T)=R_{x y}(T) / R_{x x}(0) \\
& R_{X X}(T)=\left\langle x_{k-1} x_{k}\right\rangle \\
& R_{X Y}(T)=\left\langle x_{k-1} Y_{k}\right\rangle
\end{aligned}
$$

The phase $\phi_{k}$ assumes all values within the range $0<\phi_{k}<2 \pi$ with the same probability, hence:

$$
p\left(\phi_{k}\right)=p\left(\phi_{k-1}\right)=1 / 2 \pi
$$

Using expressions $(6.5),(6.7)$ and (6.8) the probability density function of the instantaneous frequency can be expressed as: 


$$
p\left(\hat{f}_{i}(k)\right)=\frac{D}{\left(1-A^{2}\right)^{3 / 2}} \cdot\left\{\left(1-A^{2}\right)^{\frac{1}{2}}+A(\pi-\arccos A)\right\}(6.9)
$$

where

$$
\begin{aligned}
& A=\rho_{x x}(T) \cos \left(2 \pi f_{i}(k)\right)+\rho_{x y}(T) \sin \left(2 \pi f_{i}(k)\right) \\
& D=i 1-\rho_{x x}{ }^{2}(T)-\rho_{x y}{ }^{2}(T) !
\end{aligned}
$$

for the interval $-0.5<\hat{f}_{i}(k)<0.5$ where $\hat{f}_{i}(k)$ is normalized with respect to the $P R F$. The density function $p\left(\hat{f}_{i}(k)\right)$ is periodic with period 1 . It can be concluded that the function only depends on the signal parameters $\rho_{x x}(T)$ and $\rho_{x y}(T)$. In general the distribution of the instantaneous frequency will differ from the spectral distribution of the signal since the last one is determined by (Angelsen, 1980b) $\rho_{x y}(n T)$ and $\rho_{x x}(n T), n=$ $\ldots-2,-1,0,1,2 \ldots$

The distribution of instantaneous frequencies of a Doppler signal contaminated by noise with a spectral distribution as depicted in fig. 6.5 can easily be computed analytically. We assume that the signal has a rectangular spectrum with a spectral density $S_{0}$ while the noise spectrum is uniform over the full frequency range with density $\mathrm{N}_{\mathrm{O}}$. Hence,

$$
\begin{aligned}
R_{X x}(0) & =\frac{1}{T} \int_{-0.5}^{0.5} S_{0}(f)+N_{0}(f) d f \\
& =S+N \\
R_{X x}(T) & =\frac{1}{T} \int_{-0.5}^{0.5}\left(S_{O}(f)+N_{O}(f)\right) \cos 2 \pi f d f \\
& =S_{0}(f)\left(\sin \left(2 \pi\left(f_{C}+B / 2\right)\right)-\sin \left(2 \pi\left(f_{C}-B / 2\right)\right)\right) / 2 \pi T
\end{aligned}
$$




$$
\begin{aligned}
& =\left(S \cos 2 \pi f_{C}{ }^{*} \sin C \pi B\right) / 2 \\
R_{X Y}(T) & =\left(S \sin 2 \pi f_{C}{ }^{*} \sin C \pi B\right) / 2
\end{aligned}
$$

In the above expressions $f_{C}$ and $B$ are the normalized central frequency and bandwidth of the Doppler signal, respectively. The factor $I / T$ is due to this normalization. The normalized correlation functions are equal to:

$$
\begin{aligned}
& \rho_{X X}(T)=S\left(\sin c \pi B^{*} \cos 2 \pi f_{C}\right) / 2(S+N) \\
& \rho_{x y}(T)=S\left(\sin c \pi B^{*} \sin 2 \pi f_{C}\right) / 2(S+N)
\end{aligned}
$$

Substituting expressions (6.11a) and (6.11b) into (6.9) yields:

$$
A=S\left(\operatorname{sinc} \pi B^{*} \cos 2 \pi\left(f_{i}(k)-f_{C}\right)\right) / 2(S+N)
$$

The probability density function will be shifted along the frequency-axis over $f_{C}$. Mapping will occur for the values of $f_{i}(k)$ that $i f_{i}(k)-f_{c} j>0.5$. The expression for $p\left(\hat{f}_{i}(k)\right)$ takes automatically care for this mapping. Depending on the signal-tonoise ratio and signal bandwidth $p\left(f_{i}(k)\right)$ will be more spread. The distribution of instantaneous frequencies is not confined to the signal bandwidth (Salz, 1974). This is demonstrated by noise with a uniform spectral distribution over the range -0.25 to $0.25\left(\mathrm{f}_{\mathrm{C}}=0\right)$. Expression $(6.11)$ changes then into:

$$
\begin{aligned}
& \rho_{X X}(T)=1 / \pi \\
& \rho_{X Y}(T)=0
\end{aligned}
$$

and expression (6.12) into:

$$
A=\cos 2 \pi f_{i}(k) / \pi
$$

Substituting expressions (6.12) and (6.13) into (6.9) reveals that $p\left(\hat{\mathrm{F}}_{i}(k)\right)$ does not vanish for $; \mathrm{f}_{i}(k) i>0.25$. 
104

A typical distribution of the instantaneous frequency is given in fig. 6.7. The dashed Iine represents the theoretical distribution according to expression (6.9) for a signal with central frequency $f_{C}=0.4$ and a bandwidth $B=0.1$. The signal-tonoise ratio in this simulation was set at $5 \mathrm{~dB}$. The normalized correlation coefficients $\rho_{x x}(T)$ and $\rho_{x y}(T)$ were obtained experimentally using simulated Doppler signals (see section 6.3)

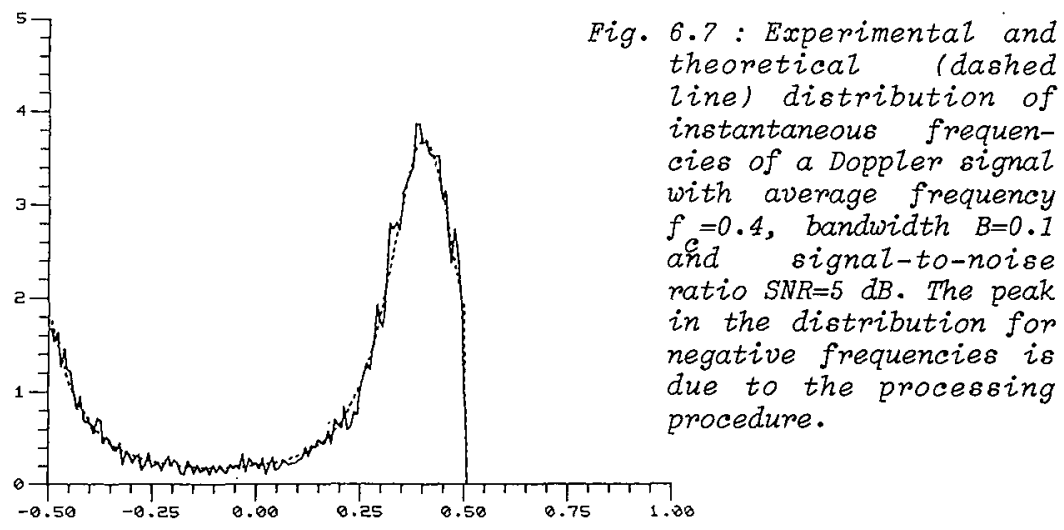

although the theoretical distribution hardly changed if expression 6.11 was used to obtain $\rho_{x x}(T)$ and $\rho_{x y}(T)$. This is not remarkable since the experimental signal spectrum hardly deviates from a rectangular distribution because of the employed loth order band-pass. The solid line in fig. 6.7 gives the observed distribution of instantaneous frequencies computed according to expression (6.4) from a simulated signal consisting of $2^{13}$ in quadrature samples. Both curves agree excellently, including the mapping of instantaneous frequencies exceeding 0.5 into the region with negative frequencies. Due to the shift over $f_{c}$ (see expression 6.12) the probability density function is truncated at $f=0.5$ and continues at $f=-0.5$.

The difference between the distribution of the instantaneous frequency of a (sampled) signal and its spectral distribution is demonstrated in fig. 6.8 where for the same signal as used in $\mathrm{fig} .6 .7\left(\mathrm{f}_{\mathrm{C}}=0.4, \mathrm{~B}=0.1, \mathrm{SNR}=5 \mathrm{~dB}\right)$ both the spectrum (dashed 1 ine) and the observed distribution of instantaneous frequen- 


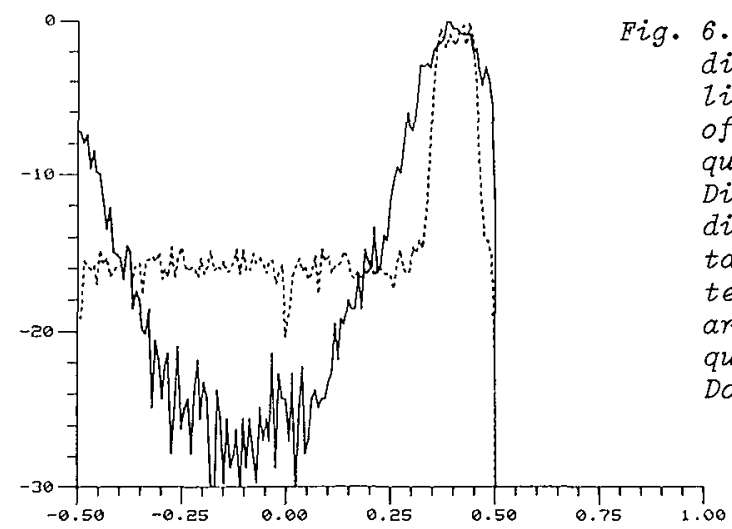

.8: Observed spectral line) and distribution of instantaneous frequencies (solid line). Disregarding mapping the distribution of instantaneous frequencies tends to concentrate around the central frequency of the generated Doppler signal.

cies (solid line) are depicted. For both curves the values are given in $d B$ with respect to the observed peak value (the observed distribution in fig. 6.7 was on a linear scale). Note that the instantaneous frequency tends to concentrate around the most dominant frequency $i . e$. the central frequency $f_{c}$.

Due to mapping of instantaneous frequencies it can be anticipated that the time-average of the instantaneous frequency will have an increasing error for increasing signal bandwidth and central frequency (Angelsen, 1979). The relative error in the time average will further increase for a decreasing signal to noise ratio. For a bandwidth of $B=0.01, B=0.1$, and $B=0.3$ respectively the relative error in the time-average of the instantaneous frequency was computed for a simulated signal as function of the central frequency $f_{C}$ for a signal-to-noise ratio of $30 \mathrm{~dB}$ (fig. 6.9a), $20 \mathrm{~dB}$ (fig. 6.9b) and $10 \mathrm{~dB}$ (fig. $6.9 \mathrm{C})$. For small bandwidths $(B=0.01$ and $B=0.1)$ the observed error is neglectable for $\mathrm{f}_{\mathrm{C}} \mathrm{i}<0.4$ and good ( $\mathrm{SNR}=30 \mathrm{~dB}$ ) or modderate $(S N R=20 \mathrm{~dB})$ signal-tonoise ratios. For a wide-band signal $(B=0.3)$ the observed relative error is substantial for almost all signal conditions. Comparing these results with those depicted in fig. 6.6 reveals that the time-discrete instantaneous frequency detector has a worse performance than the 


\section{6}

time-discrete directional zero crossing detector except for wide-band signals with a central frequency close to zero.
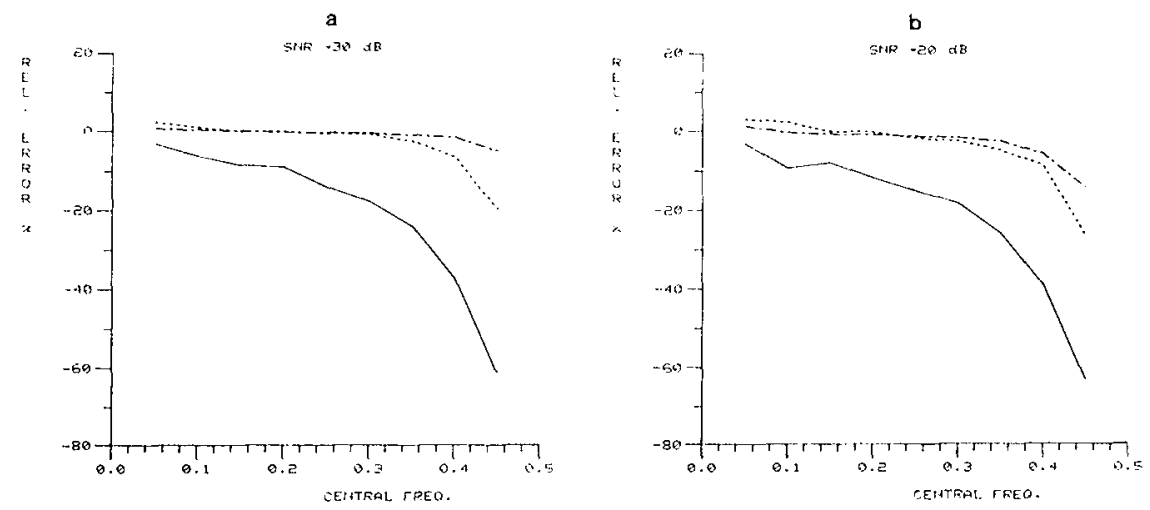

Fig. 6.9: The fractional error in the output of a frequency detector based on the time-average of the instantaneous frequencies. The observed error increases with increasing bandwidth of the Doppler signal and/ or decreasing SNR.

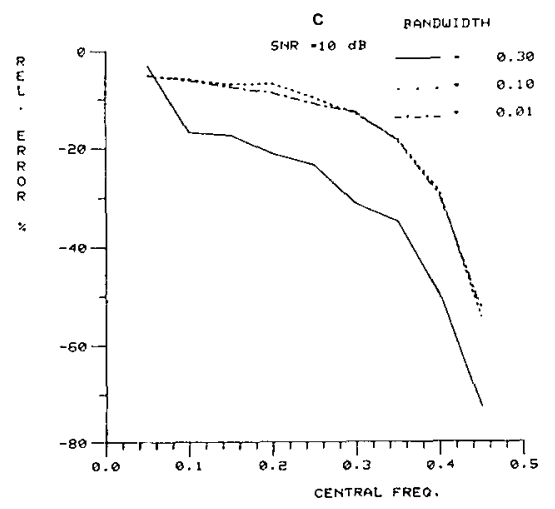

The situation will change dramatically if mapping of instantaneous frequencies is detected and corrected for. As pointed out in section 6.5 a simple algorithm to detect mapping is to consider the difference between the detected instantaneous frequency and its time-average. For correction an integral number of the PRF should be added to the detected instantaneous frequency in such a way that the difference between corrected instantaneous frequency and the time-average becomes less than $\mathrm{RRF} / 2$. The corrected instantaneous frequency is then used as the input for a moving average filter (i.e. a low-pass filter) 
to compute the time-average. As low-pass filter a digital lowpass filter as introduced in section 5.2 can be used. The filter-constant $K$ determines the response time of the low-pass filter. We will deal with the arguments for the selection of $k$ in sections 6.7 and 6.8 . The above algorithm is applied for the same signal as used for $f i g .6 .7$ ( $f_{C}=0.4, B=0.1, S N R=5 d B$ ). The result for $K=1 / 32$ is depicted in $f i g .6 .10$ (solid curve). The

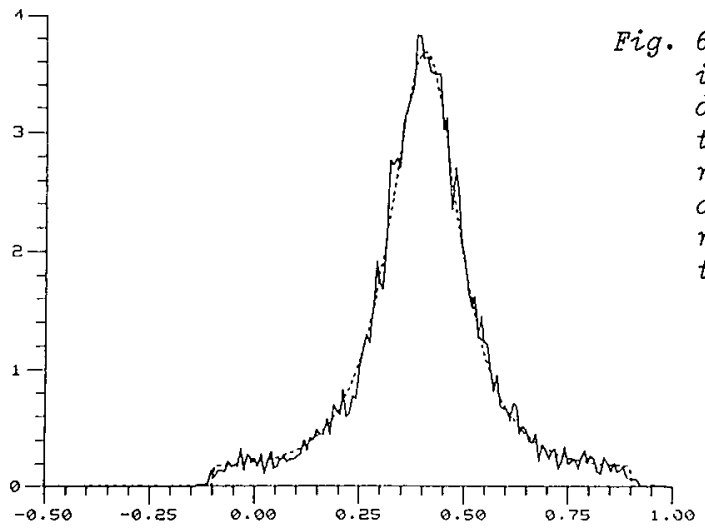

6.10: Experimental (solid line) and theoretical distribution of instantaneous frequencies corrected for aliasing. The distribution is symmetrically around the central frequency.

dashed curve gives the theoretical distribution of instantaneous frequencies based on expression (6.9) but without mapping. Again both curves are in excellent agreement. The distribution is symmetric around the central frequency as predicted in section 6.5. Hence, the error in the time-average due to noise and due to mapping will be reduced considerably. In the next section the effect of correction on the relative error in the estimated average frequency for various signal conditions will be discussed in more detail.

\subsection{RELATIVE ERROR AND STANDARD DEVIATION OF THE TIME AVERAGE OF THE INSTANTANEOUS FREQUENCY}

As stated in the previous section correction for aliasing of the instantaneous frequency will result in a symmetrical distribution of the instantaneous frequency around its central frequency, at least for signals with a symmetric spectral dis- 
108

tribution (fig. 6.5). It can therefore be anticipated that the bias in the estimator for the central frequency due to noise
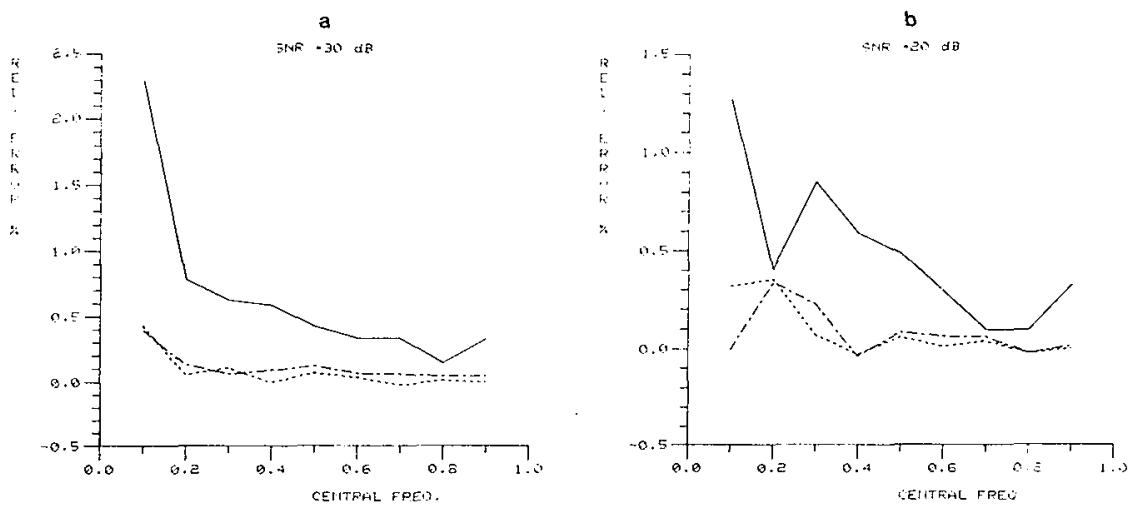

Fig. 6.11: The fractional error in the output of an estimator based on the instantaneous frequency with correction for aliasing. The error is negligible liess than $2 \%)$ for a wide range of central frequencies, bandwidths and signalto-noise ratios.

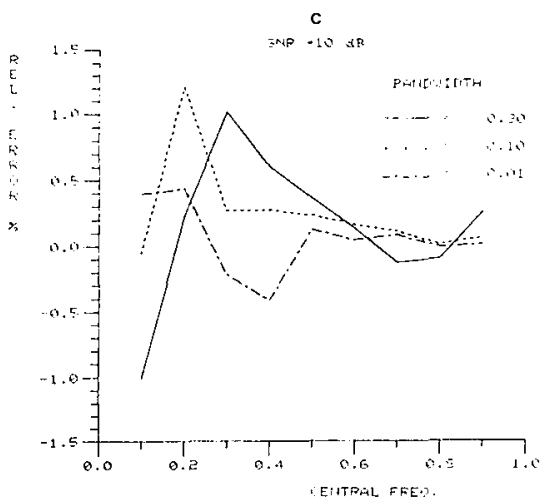

will vanish. In fig. 6.11 the relative error is depicted as function of the central frequency (ranging from 0.05 to 0.95 in steps of 0.05$)$ for three bandwidths of the signal $(B=0.3, B=0.1$ and $B=0.01$ ) and three signal-to-noise ratios ( $S N R=30 \mathrm{~dB}$, $S N R=20$ $d B$ and $S N R=10(d B)$. The result is based on the processing of 10.000 in quadrature samples. The filter constant $\mathrm{K}$ in the digital lowpass filter of the first order used to obtain the time average of the corrected instantaneous frequencies was set at $K=1 / 32$. As can be observed the relative error is surprisingly small for all of the above mentioned signal conditions (within 
$1 \%$ ) except for $f_{C}=0.05$ and a wide bandwidth ( $B=0.3$ ) where the relative error is in the order of $2.5 \%$. These results demonstrate that the instantaneous frequency detector with correction is superior with respect to an instantaneous frequency detector without correction (fig. 6.9) and a directional timediscrete zero crossing detector (fig. 6.6).
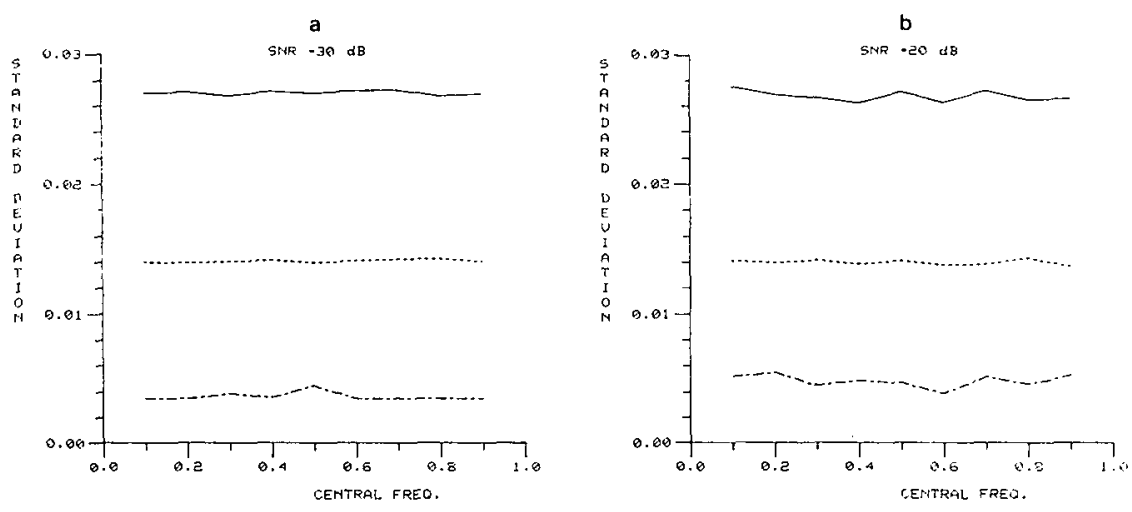

Fig. 6.12: The standard deviation of the output of an estimator based on the corrected instantaneous frequency increases with an increasing bandwidth of the similated signal. This effect will be especially prominent if the bandwidth of the Doppler signals is narrow and if the signal-to-noise ratio is poor.

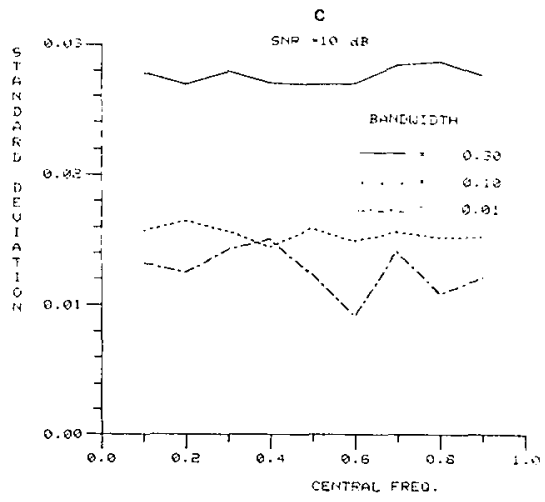

The excursions of the time-average of the corrected instantaneous frequency around its mean depend on the width and the shape of the distribution of the corrected instantaneous frequencies, hence on the signal-to-noise ratio, the spectral distribution of the simulated signal and the response time of the digital low-pass filter to obtain the time-average. In fig. 6.12 the observed standard deviation is given for the same 
110

signal- and processing conditions as for fig. 6.11. For good ( $S N R=30 \mathrm{~dB}$ ) and moderate (SNR=20 dB) signal-to-noise ratios the standard deviation is rather independent of the central frequency. For signals with a small bandwidth a decreasing signalto-noise ratio increases the standard deviation because of an increasing width of the distribution of instantaneous frequencies. This effect can be observed in fig. $6.12 \mathrm{~b}$ for $\mathrm{B}=0.01$ and in fig. $6.12 \mathrm{C}$ for $\mathrm{B}=0.01$ and for $\mathrm{B}=0.1$ as well.

In fig. 6.13 the observed relative error and standard deviation is given as function of the signal-to-noise ratio (ranging from $-5 \mathrm{~dB}$ to $30 \mathrm{~dB}$ in steps of $5 \mathrm{~dB}$ ) for a signal with a fixed
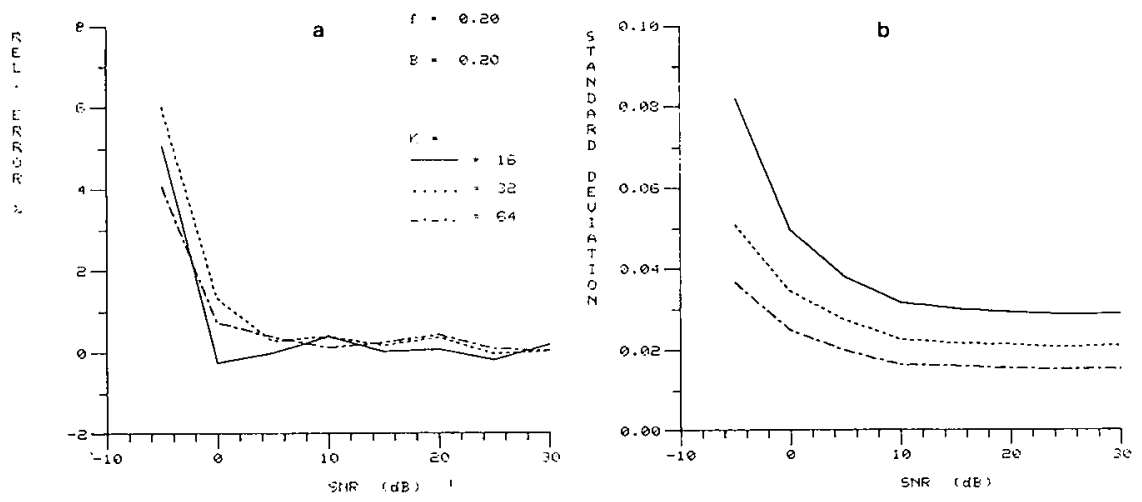

Fig. 6.13: The fractional error and standard deviation in the output of a frequency estimator based on the corrected instantaneous frequency as function of the signal-tonoise ratio (SNR) for $f=0.2$ and $B=0.2$. The response time of the low-pass filter used to compute the average of the corrected instantaneous frequency is characterized by its filter constant $K=1 / 16, K=1 / 32$ and $K=1 / 64$.

bandwidth $(B=0.2)$ and fixed central frequency $\left(f_{C}=0.2\right)$. The time-average is obtained using three filter constants $(K=1 / 16$, $K=1 / 32$ and $K=1 / 64)$. For a SNR of $0 \mathrm{~dB}$ or better the relative error falls again within $1 z$ independent of the choice of the filter constant. For a poor signal-to-noise ratio (-5dB) an increase in relative error can be observed (up to approximately 58 ). This can be attributed to the problem to trace the most 
dominant instantaneous frequencies. For a vanishing signal the normalized correlation coefficients $P_{x x}(T)$ and $P_{x y}(T)$ will tend to zero and a uniform distribution (see expression 6.9) for the instantaneous frequency will result. Under this condition no central frequency is present and the correction algorithm will result in false mapping. The time-average of the instantaneous frequency will follow a random course. This phenomenon becomes more important for a SNR of $-6 \mathrm{~dB}$ and becomes dominant for $a$

\begin{tabular}{|c|c|c|c|c|c|c|c|c|}
\hline \multirow[b]{2}{*}{ B } & \multirow[b]{2}{*}{$f_{c}$} & \multirow[b]{2}{*}{$\begin{array}{l}\text { SNR } \\
(\alpha B)\end{array}$} & \multicolumn{3}{|c|}{$\mathrm{K}=1 / 16$} & \multicolumn{3}{|c|}{$K=1 / 32$} \\
\hline & & & $s\left(\hat{f}_{i}\right)$ & 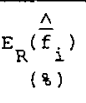 & $s\left(\hat{\bar{f}}_{i}\right)$ & $s\left(\hat{f}_{i}\right)$ & $\begin{array}{c}E_{R}\left(\overrightarrow{\hat{f}}_{i}\right) \\
(z)\end{array}$ & $s\left(\hat{\bar{E}}_{i}\right)$ \\
\hline \multirow{3}{*}{0.1} & 0.1 & $\begin{array}{r}-10 \\
0 \\
10 \\
20 \\
30\end{array}$ & $\begin{array}{c}- \\
0.218 \\
0.113 \\
0.063 \\
0.054\end{array}$ & $\begin{array}{r}- \\
6.3 \\
0.9 \\
0.2 \\
-0.2\end{array}$ & $\begin{array}{c}- \\
0.047 \\
0.023 \\
0.020 \\
0.020\end{array}$ & $\begin{array}{l}0.301 \\
0.216 \\
0.112 \\
0.063 \\
0.054\end{array}$ & $\begin{array}{r}53.8 \\
2.1 \\
0.7 \\
0.1 \\
-0.3\end{array}$ & $\begin{array}{l}0.124 \\
0.030 \\
0.016 \\
0.014 \\
0.014\end{array}$ \\
\hline & 0.3 & $\begin{array}{r}-10 \\
0 \\
10 \\
20 \\
30\end{array}$ & $\begin{array}{l}0.323 \\
0.217 \\
0.110 \\
0.064 \\
0.054\end{array}$ & $\begin{array}{r}6.1 \\
1.7 \\
0.4 \\
-0.0 \\
-0.0\end{array}$ & $\begin{array}{l}0.173 \\
0.050 \\
0.022 \\
0.020 \\
0.020\end{array}$ & $\begin{array}{l}0.296 \\
0.215 \\
0.110 \\
0.064 \\
0.054\end{array}$ & $\begin{array}{r}6.1 \\
1.9 \\
0.4 \\
-0.0 \\
-0.0\end{array}$ & $\begin{array}{l}0.114 \\
0.034 \\
0.015 \\
0.014 \\
0.014\end{array}$ \\
\hline & 0.6 & $\begin{array}{r}-10 \\
0 \\
10 \\
20 \\
30\end{array}$ & $\begin{array}{l}0.334 \\
0.219 \\
0.111 \\
0.064 \\
0.055\end{array}$ & $\begin{array}{r}-0.5 \\
0.3 \\
0.2 \\
0.0 \\
-0.0\end{array}$ & $\begin{array}{l}0.189 \\
0.051 \\
0.022 \\
0.020 \\
0.020\end{array}$ & $\begin{array}{l}0.293 \\
0.217 \\
0.111 \\
0.064 \\
0.055\end{array}$ & $\begin{array}{r}0.7 \\
0.1 \\
0.2 \\
0.0 \\
-0.0\end{array}$ & $\begin{array}{l}0.098 \\
0.033 \\
0.016 \\
0.015 \\
0.014\end{array}$ \\
\hline \multirow{3}{*}{0.3} & 0.1 & $\begin{array}{r}-10 \\
0 \\
10 \\
20 \\
30\end{array}$ & $\begin{array}{c}- \\
0.230 \\
0.153 \\
0.132 \\
0.129\end{array}$ & $\begin{array}{l}- \\
2.5 \\
2.9 \\
4.0 \\
3.0\end{array}$ & $\begin{array}{c}- \\
0.059 \\
0.038 \\
0.037 \\
0.037\end{array}$ & $\begin{array}{l}0.307 \\
0.228 \\
0.152 \\
0.129 \\
0.129\end{array}$ & $\begin{array}{r}27.4 \\
7.0 \\
2.5 \\
4.2 \\
2.4\end{array}$ & $\begin{array}{l}0.133 \\
0.043 \\
0.026 \\
0.026 \\
0.027\end{array}$ \\
\hline & 0.3 & $\begin{array}{r}-10 \\
0 \\
10 \\
20 \\
30\end{array}$ & $\begin{array}{c}- \\
0.228 \\
0.153 \\
0.132 \\
0.129\end{array}$ & $\begin{array}{l}- \\
0.9 \\
0.1 \\
0.7 \\
0.8\end{array}$ & $\begin{array}{c}- \\
0.053 \\
0.038 \\
0.038 \\
0.037\end{array}$ & $\begin{array}{l}0.295 \\
0.226 \\
0.153 \\
0.132 \\
0.129\end{array}$ & $\begin{array}{r}0.9 \\
-0.1 \\
0.2 \\
0.7 \\
0.8\end{array}$ & $\begin{array}{l}0.102 \\
0.036 \\
0.028 \\
0.028 \\
0.027\end{array}$ \\
\hline & 0.5 & $\begin{array}{r}-10 \\
0 \\
10 \\
20 \\
30\end{array}$ & $\begin{array}{l}0.315 \\
0.229 \\
0.154 \\
0.131 \\
0.129\end{array}$ & $\begin{array}{r}-5.2 \\
0.8 \\
0.6 \\
0.4 \\
0.5\end{array}$ & $\begin{array}{l}0.156 \\
0.058 \\
0.042 \\
0.038 \\
0.038\end{array}$ & $\begin{array}{r}0.290 \\
0.227 \\
0.154 \\
0.131 \\
0.128\end{array}$ & $\begin{array}{r}-1.2 \\
0.8 \\
0.4 \\
0.4 \\
0.5\end{array}$ & $\begin{array}{l}0.090 \\
0.041 \\
0.031 \\
0.027 \\
0.027\end{array}$ \\
\hline
\end{tabular}

Table 6.1: Observed standard deviation of the distribution of the instantaneous frequency and the observed standard deviation and fractional error in the estimated average (after correction) for 2 filter constants $(K=1 / 16$ and $K=1 / 32)$ as function of the bandwidth $(B)$ and center frequency $(f)$ of the simulated Doppler-signal and the signal-to-noise ratio (SNR). 
112

SNR of $-10 \mathrm{~dB}$ or less. For these values of the SNR the correction algorithm failed. The increasing standard deviation for low signal-to-noise ratios (fig. 6.13b) is due to the widening of the distribution of the instantaneous frequency. For values of the SNR of $10 \mathrm{~dB}$ or better the variance can be approximated by $B K / 12$ where $1 / K$ represents the normalized response time of the low-pass filter. This is equivalent to the expression for the standard deviation as derived by Gerzberg (Gerzberg,1980). It would be, therefore, advantageous to select $K$ as low as possible. However, a low $K$ affects the ability of the correction algorithm to adjust for changing signal conditions (section 6.8). In table 6.1 the standard deviation of the corrected instantaneous frequency and of its time-average is listed for two values of the filter constant ( $K=1 / 16$ and $K=1 / 32)$ and for various signal conditions. For low signal-to-noise ratios (SNR=-10 $\mathrm{dB}$ ) and $\mathrm{K}=1 / 16$ the correction mechanism failed to arrive at a correct estimate for the central frequency $\left(f_{C}=0.1\right)$. Low values of the filter constant have a benificial effect on the standard deviation of the corrected instantaneous frequency (the faedback loop incorporated in the correction mechanism affects the distribution of the instantaneous frequency). The selection of the central frequency (for a constant SNR and bandwidth) does not affect the result.

\subsection{TRACKING BEHAVIOUR OF THE CORRECTION MECHANISM}

In the previous section the correction mechanism was evaluated under stationary conditions (fixed SNR, bandwiath and central Erequency). However, in the practical situation these conations are a function of time and recoraing conditions (site of measurement). The correction mechanism should be able to adjust dynamically to a change in signal parameters.

In fig. 6.14 the transfar function of the filter to reject low Doppler frequencies with high amplitude is depicted. Due to the sample procedure the transfer function is periodic with the 
pulse repetition frequency. Therefore, a dip in the transfer function will show up at integral numbers of the pulse repetition frequency. Because of the correction mechanism only fre-

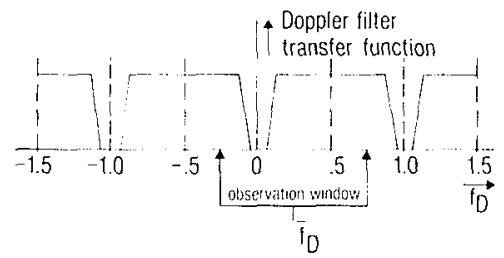

Fig. 6.14: A frequency detector based on the corrected instantaneous frequency considers only frequencies within a given observation window (width equal to 1) around the average frequency $\overline{f_{D}}$.

quencies within the observation window will be observed. Doppler frequencies outside this window will be mapped within this window. That is why the distribution of the Doppler signal should extend over a range of less than the PRF (otherwise no distinction can be made between aliased and non-aliased frequencies). The dip in the frequency spectrum due to the highpass characteristics of the Doppler filter will affect the spectral distribution of the corrected instantaneous frequency but a dip will not show up (unless the Doppler signal has a very narrow bandwidth). For a Doppler signal with a given bandwidth and increasing central frequency the distribution of the instantaneous frequency will temporarily widen as the central Erequency passes over the rejection zone. Instabilities in the correction mechanism may appear if the central frequency is at an integral number of the PRF. The situation will be worse if the Doppler signal has a large bandwidth and/or the response time of the averaging filter is short. A larger response time will reduce the standard deviation of the time-average of the instantaneous frequency and enhance stability. However, a large rasponse time will introduce a phase-lag between the detected instantaneous frequencies and their time-average. At higher rates of change of the central frequency this phase-lag introduces a difference between the time-average and actual central frequency. This difference is at the expense of the interval over which the instantaneous Erequencies can be corrected adequately. 
114

In fig. 6.15 the response of the correction mechanism is given for a signal consisting of 9500 samples with a fixed bandwidth $(B=0.3)$ and a central frequency changing quadratically with time (dashed line). The total time is equivalent to one second. The rate of change is expressed as the number of
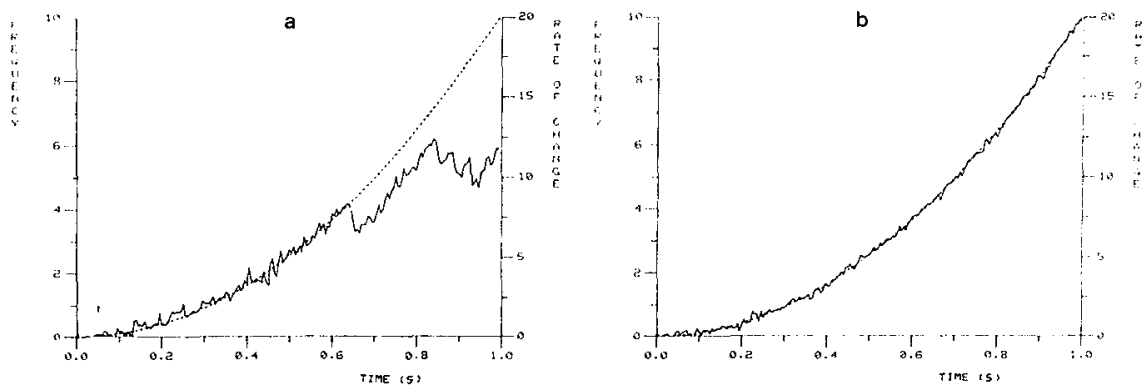

Fig. 6.15: The tracking behaviour of the frequency estimator for a Doppler signal with fixed bandwidth $(B=0.3)$ and a central frequency changing quadratically with time (horizontal axis). Fig. $6.15 a$ gives the result for a filter constant $K=1 / 18$ and $S N R=-5 d B$. Tracking is lost for a rate of change of $7 P R E / 8$. If the SNR is set at 0 dB the system is able to track the central frequency even if the rate of change goes up to $20 \mathrm{PRF} / \mathrm{s}$ ( $\mathrm{fig}$. $6.15 b)$. A reduction in the fluctuations in the time average can be obtained by increasing the response time of the moving average filter (fig. 6.15c).

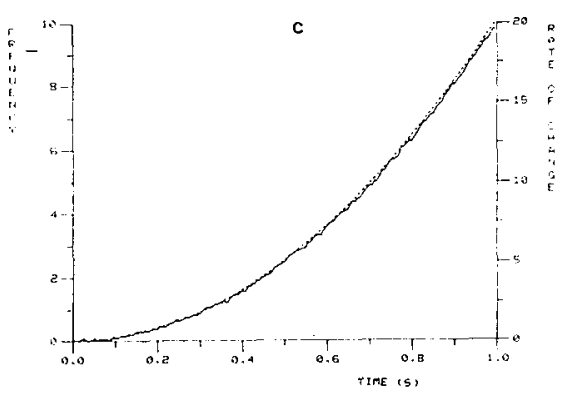

PRF per second i.e. a rate of change of 10 is equivalent to a change in the central frequency of 10 times the PRF per second. In the early systolic phase the peak velocity of the blood flow as recorded, for example, in the common femoral artery may change $60 \mathrm{~cm} / \mathrm{s}$ within $80 \mathrm{msec}$. For a $6 \mathrm{MHz}$ Doppler system with a PRF of $9.5 \mathrm{kHz}$ and an angle of observation of $45^{\circ}$ this is equivalent to a rate of change in the order of $5 \mathrm{PRF} / \mathrm{s}$. In cardiac applications the rate of change can easily reach 10 $\mathrm{PRF} / \mathrm{s}$ even if a lower emission frequency is taken into account. 
The correction mechanism should be able to track these rates of change. A short response time of the moving averager (fig. $6.15 a)$, where the filter constant is set at $K=1 / 8$, and a poor signal-to-noise ratio ( SNR $=-5 \mathrm{~dB}$ ) causes that tracking is lost at a rate of change of $7 \mathrm{PRF} / \mathrm{s}$. But even at lower rates of change large fluctuations in the time-average of the corrected instantaneous frequency appear. These excursions are considerably reduced for a somewhat better signal-to-noise ratio ( $S N R=0$ $\mathrm{dB}$ ) and the system is able to track the center frequency of the Doppler signal up to a rate of change of at least $20 \mathrm{PRF} / \mathrm{s}$ (fig. 6.15b). Increasing the response time of the moving averager (setting the filter constant at $K=1 / 32$ ) gives a further reduction in the fluctuations in the time average although at high rates of change a phase-lag between the detected timeaverage (solid line) and the theoretical central frequency (dashed line) can be observed (fig. 6.15c).

In fig. 6.15 the central frequency of the signal reaches 10 times the PRF. It is tempting to conclude that the frequency range of a pulsed Doppler system with an instantaneous frequency detector with correction is unbounded. However, the bandwidth of the Doppler signal (disregarding frequency dispersion due to the observed velocity distribution) is related to the central frequency due to the transit time effect. Taking a Doppler bandwidth of 0.5 PRF as a maximum (for larger bandwidths the spectral distribution of the instantaneous frequency becomes more and more uniform) and assuming the following relation between bandwidth and central frequency

$$
B=0.2 f_{C}
$$

then the following relation for the maximum central frequency $f_{c, \max }$ holds:

$$
\begin{aligned}
& 0.2 f_{c, \max }=0.5 \\
& E_{C, \max }=2.5
\end{aligned}
$$


116

Although the application of the correction algorithm allows for a more flexible selection of the pulse repitition frequency the PRF still limits the maximum velocity that can be detected but this limitation depends now also on the dimensions of the sample volume. If the bandwidth of the Doppler signal increases due to velocity distribution within the sample volume then the maximum center frequency will be lower accordingly. It can be concluded from fig. 6.15 that for a wide range of signal-tonoise ratios ( $S N R=-5 \mathrm{~dB}$ represents a worst case) the tracking procedure functions flawless for $\mathrm{K}=1 / 16$ or $\mathrm{K}=1 / 32$ even for Doppler signals with a relative wide bandwidth $(B=0.3)$. Replacement of the directional time-discrete zero crossing detector by a time-discrete instantaneous frequency detector with correction for aliasing will improve the performance of a multigate pulsed Doppler system with serial data-processing.

\subsection{HARDWARE REALIZATION OF THE INSTANTANEOUS FREQUENCY DETECTOR}

In the multi-gate pulsed Doppler system with serial data processing the detection of the average Doppler frequency within the observed sample volumes is achieved by computing a moving time average of the zero crossings. The application of a detector based on correction of the detected instantaneous frequency requires the implementation of another frequency detector circuit. Since the instantaneous frequency is equivalent to the rate of change in the phase of the in quadrature Doppler signals as observed in successive samples the zero crossing detector has to be converted into a phase-detector able to compute

$$
o_{i}=\arctan \left(y_{k} / x_{k}\right)
$$

where $\rho_{i}$ is periodic with a period of 2 . The instantaneous phase $\rho_{i}$ is then automatically expressed with respect to the PRF. The quadrant in which the phase vector is located can be 


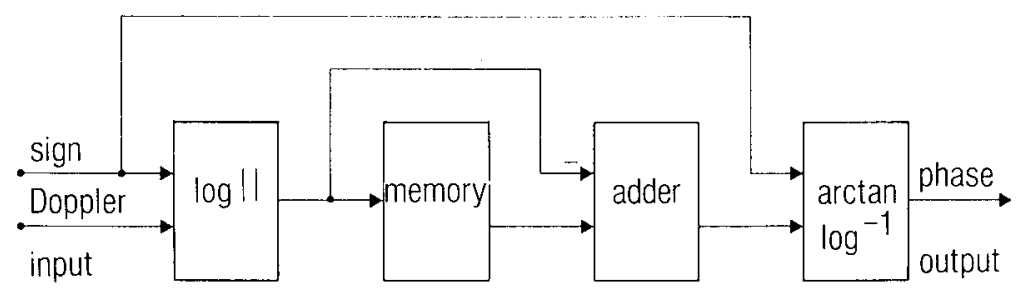

Fig. 6.16: Block-diagram for an instantaneous phase detector. The calculation of the ratio of the in quadrature components is facilitated by the conversion of the instantaneous signal amplitude into logarithmic form.

retrieved from the signs of the in quadrature Doppler signals. The sign bits provide 2 bits of information for the instantaneous phase $\rho_{i}$. The computation of the arctan can, therefore, be based upon the ratio of the absolute value of the in quadrature components. In $\mathrm{fig}$. 6.16 the basic block diagram for the instantaneous phase detector is depicted. To calculate the ratio in a fast way the absolute value of the in quadrature components are converted into logarithmic form. Since the Doppler signals are on an 8-bit base (including sign) the logarithm (base 2) of the absolute value will give 3 bits before the binary point. Four bits are added to increase the accuracy of the conversion $\left({ }^{2} \log 0\right.$ is set to zero). Since the in quadrature components arrive in serial form at the input of the phase detector a register is incorporated to save sign and the 7-bits logarithmic value of the first component while the conversion of the second one takes place. The sign of the difference $x$ of both logarithmic values indicates, in combination with the signs of the quadrature components, the octant in which the phase vector is located ( 3 bits of information) while a PROM takes care of the conversion arctan $2^{x}$. The output of the PROM will change most rapidly if $x$ is around 0 . Since $x$ has an accuracy of 3 bits behind the binary point (one bit is lost due to subtraction) the accuracy of the phase detection can be estimated by computing the result for $x=0$ (phase angle $45^{\circ}$ ) and $x=1 / 8$ (phase angle $47.48^{\circ}$ ). Expressing the phase-angle on a 7 bit phase ( 3 bits provided by the sign-information and 4 bits 
118

by the PROM) will give an angular resolution of $\pm 360^{\circ} /\left(2 \times 2^{7}\right)=$ $\pm 1.4^{\circ}$ which is in accordance with the estimated resolution. Of course, this angular resolution is only obtained for larger amplitudes of the Doppler signal. A single $256 \times 4$ bit PROM is needed for the conversion: 6 input bits for the difference, 1 input bit for the sign of $x$ and 1 input bit to indicate whether the phase vector is located in the right or left half of the phase plane (this information is provided by the sign of the second component of the in quadrature signals). The instantaneous phase detector output on a 7-bit base is scaled such that for increasing angle the output ranges from 0 to 2 ( 2 excluded).

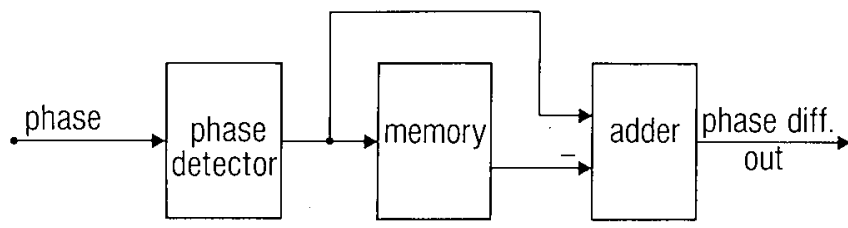

Fig. 6.17: In order to obtain the change in phase in two consecutive processing cycles the output of the instantaneous phase detector is temporarily stored in a memory.

The calculation of the instantaneous frequency involves subtraction of the detected instantaneous phases (modulo l) in subsequent processing cycles, requiring a memory to retain the detected phase (fig. 6.17). The computed phase difference is then equivalent to an instantaneous frequency in the range from $-\mathrm{PRF} / 2$ to $\mathrm{PRF} / 2$ (PRF/2 excluded).

To execute the correction algorithm a moving time average of the instantaneous frequency is needed, requiring a low-pass filter. A cut-off frequency in the order of $20 \mathrm{~Hz}$ demands for a small filter constant $\left(K=2^{-6}\right)$ for a PRF of $9.5 \mathrm{kHz}$. However, the computer simulations indicated that such a low-pass filter will affect the tracking capability unacceptably (see section 6.8). That is why low-pass filtering is executed in 2 steps. The first step involves low-pass filtering with a relative high 
cut-off freguency $(K=1 / 16)$ and the implementation of the correction algorithm while in the second step the output of the first low-pass filter is again low-pass filtered (to achieve a further reduction in variance). The cut-off frequency of a digital filter can be manipulated with the filter constant and/or the input frequency. Activating the second filter at a fixed rate of $1200 \mathrm{~Hz}$ gives for $\mathrm{K}=1 / 16$ or $\mathrm{K}=1 / 8$ a cut-off frequency of $12 \mathrm{~Hz}$ and $25 \mathrm{~Hz}$, respectively.

The subtraction (fig. 6.18) to detect the difference between input and the time-average is an integral part of a digital filter (cf. fig. 5.5). Extending the word length of the time average with 3 additional bits allows for a time-average in the range from $-7 / 2$ to $7 / 2$ times the PRF. The deviation of the input from the time-average of the instantaneous frequency will be within the same range. The least significant bit of the 4 bits preceding the binary point (including sign bit) provides the information to execute the correction algorithm. If this

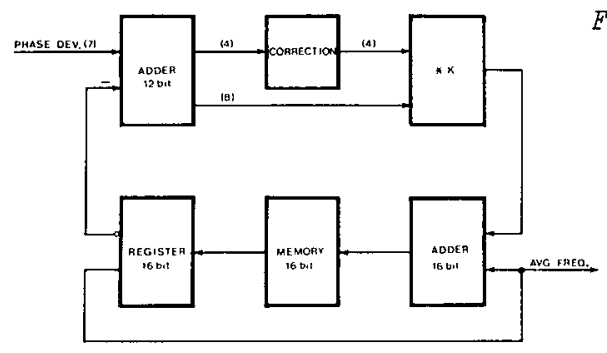

Fig. 6.18: Block-diagram for the correction of the detected change in phase. The correction involves only a test on the additional bits in the difference between input (phase deviation) and the time average of the (corrected) vaiues.

bit is zero the 4 most significant bits are set to zero, otherwise to 1. The corrected deviation will be in the range of -1 to 1 ( 1 excluded) equivalent to a difference of -PRF/2 to $\mathrm{PRF} / 2$. Figure 6.19 clarifies that the action based upon the value of the bit preceding the binary point results in proper correction. In this figure the difference between input and time-average is depicted as function of the time-average. The deviation is in the range $n * P R F / 2$ to $(n+1) \star P R F / 2$ where $n$ is either an odd or an even integer (this information is provided 
120

by the bit preceding the binary point). Setting the 4 most significant bits equal to the bit preceding the binary point results in a proper shift of the deviation over an integral number of PRF.

Fig. 19 : The value of
the bit preceding the
binary point in the
difference between
the detected frequen-
cy and the time-aver-
age of the corrected
values determines
whether the corrected
difference should be
positive (vertical
lines) or negative
(horizontal lines).

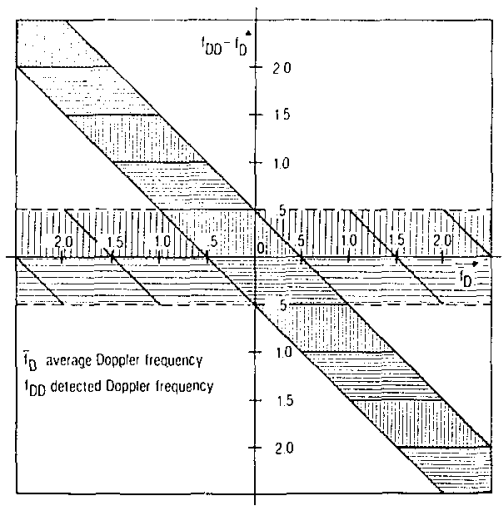

Both low-pass filters (the first one for automatic correction of the instantaneous frequency, the second one to obtain the time average with low variance) can be folded into each other (fig. 6.20), requiring an additional register and an input selector. The control signal indicates what signal is presently processed. Combining both low-pass filters in this

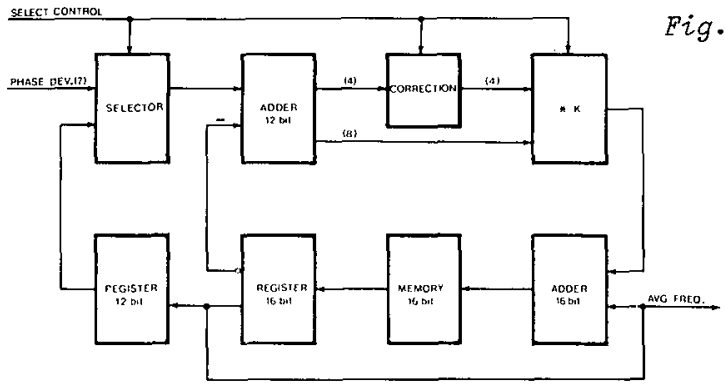

ig. 6.20: To reduce the variance in the estimator output without degeneration of the tracking behaviour the detection is performed in two steps (cf. fig. 6.18). The first step involves the short term calculation of time average employed in the correction algorithm. In the second step the long term average of the result of the first step is computed.

way only demands for doubling the number of available memory locations. Taking the input rate of the first filter at PRF=9.5 $\mathrm{kHz}$ will give for $\mathrm{k}=1 / 16$ a cut-off frequency in the order of 
$100 \mathrm{~Hz}$. This is well below the maximum signal frequency for the second low-pass filter which is $600 \mathrm{~Hz}$ for a processing rate of $1200 \mathrm{~Hz}$.

The implementation of the correction algorithm extends the range over which Doppler frequencies can be detected unambiguously. Adding 3 additional bits, as is done in the hardware realization, allows for a maximal Doppler frequency that can be processed properly of 7 times $P R F / 2$ which is $33.25 \mathrm{kHz}$ for a PRF of $9.5 \mathrm{kHz}$. A drawback, however, is that due to high-pass filtering of the Doppler signals the processed Doppler signal spectrum will show dips around an integral number of the PRF (fig. 6.14). If the Doppler spectrum is very narrow then it may happen that at integral numbers of the PRF signal information is lost. Broadening of the signal spectrum due to transit time effects will make it unlikely that narrow Doppler spectra occur at higher average Doppler frequencies.

In the absence of true Doppler signals the distribution of the detected instantaneous frequencies will be uniform over the frequency range $-\mathrm{PRF} / 2$ to $\mathrm{PRF} / 2$. The correction mechanism will fail for such a distribution. Therefore, an amplitude detector is incorporated in the phase-detector. A detected phase is considered true only if the envelope exceeds a given threshold. The corrected instantaneous frequency will have its computed value if the detected instantaneous phase in 2 subsequent processing cycles is true, otherwise it will be set to zero. The threshold is adjusted so that due to noise only the velocity output just start to fluctuate. The incorporation of the level detector ensures that in case of wrong correction the velocity output automatically returns to its true value if the amplitude of the Doppler signals vanishes. However, this condition is not met if in the diastolic phase the blood flow velocity does not return to zero. An additional protection against the detection of average Doppler frequencies differing an integral number of the PRF from the actual value is achieved by incorporating a 
check on the velocity outputs of adjacent sample gates. Assuming that the first sample gate is located outside or near the blood stream the Doppler frequency within the first sample volume will be always within the frequency range of $-P R F / 2$ to $\mathrm{PRF} / 2$. If the correction algorithm leads to the detection of frequencies outside this range then the value is automatically reset to values within this region by adding or subtracting an integral number of the PRF. It is furthermore assumed that no sharp boundaries in the velocity pattern will occur. In that condition it is unlikely that the detected average Doppler frequency in adjacent sample volumes differs more than $\mathrm{PRF} / 2$. However, if larger differences are observed then the observed frequency is automatically corrected in such a way that the frequency will fall again within the anticipated range. This is achieved by adding (for a negative average) or subtracting (for a positive average) a suitable number of the PRF. In this way unlikely discontinuities in the observed velocity pattern along the ultrasound beam are avoided.

Sampling the Doppler signal with a sample frequency less than twice the maximum Doppler frequency causes an audio output that does not reflect the spectral composition of the Doppler signal. Contradictory situations can occur, e.g. an increasing velocity output while the perceived Doppler frequencies decrease. In principle it is possible to restore the audio signal taking into account the detected average Doppler frequency. However, we opted for a simpler method to synthesize the audio output. The output of the low-pass filter used for the correction of the instantaneous frequencies is employed as input signal of a voltage controlled oscillator, adjusted so that the oscillator frequency corresponds to the short-term average Doppler frequency. The instantaneous envelope of the Doppler signal (provided by the envelope detector incorporated in the phase detector) is used to modulate in amplitude the oscillator output. The audio output generated in this way does not reflect the true Doppler signal (and can not be used for frequency 
analysis) but it provides global information about the Doppler signal amplitude and frequency.

The correction algorithm and the audio signal synthesizer was evaluated, using a string target driven by a DC-motor. The velocity of the string as function of time (fig. 6.21 bottom trace) was recorded by a tachometer. No efforts are made to simulate a smooth velocity waveform. The top trace in fig. 6.21 gives the detected average velocity without (left) and with (right) correction. Both curves are scaled with respect to each other so that they can be compared rightaway. The peak velocity with respect to the probe (mounted under an angle of $45^{\circ}$ ) is $1.25 \mathrm{~m} / \mathrm{s}$ ( $P R F=9.5 \mathrm{kHz}$ ) while the velocity changes direction twice a second. For low velocities the detected velocity waveform follows the bottom trace but at increasing velocities a

Fig. 6.21: The velocity of a string target (bottom trace) as detected with a pulsed Doppler system (top trace) with (right) and without (left) correction for frequency aliasing in the detected instantaneous frequencies. The level $F_{N}$ indicates the Nyquist frequency. The frequency of the velocity waveform is $1 \mathrm{~Hz}$.
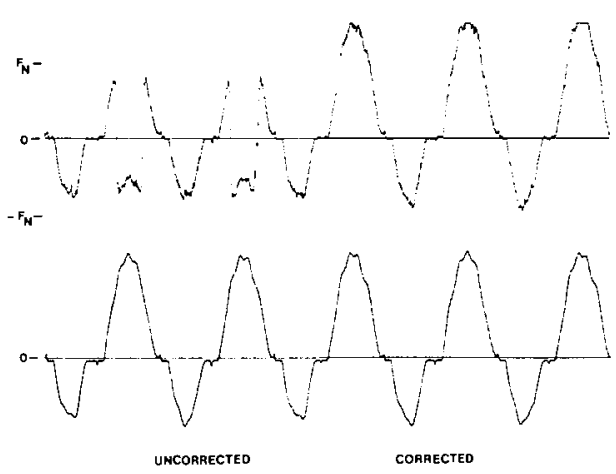

deviation can be observed (especially apparent for negative velocities) which is in accordance with section 6.6. Due to aliasing high velocities are falsely detected explaining the discontinuous velocity waveform. Incorporation of the correction algorithm restores the original waveform. The fluctuations at higher velocities are due to widening of the Doppler spectrum due to the transit time effect and instabilities of the string target (lateral motion induced by vibrations and by the knot in the string). Fig. 6.22 (left) gives the non-directional sonogram of the original Doppler signal (showing aliasing) and 


\section{4}

of the synthesized audio signal (right) with implementation of the correction algorithm (the line indicates the position of the Nyquist frequency). The widening of the spectrum at higher central frequencies can be clearly observed.
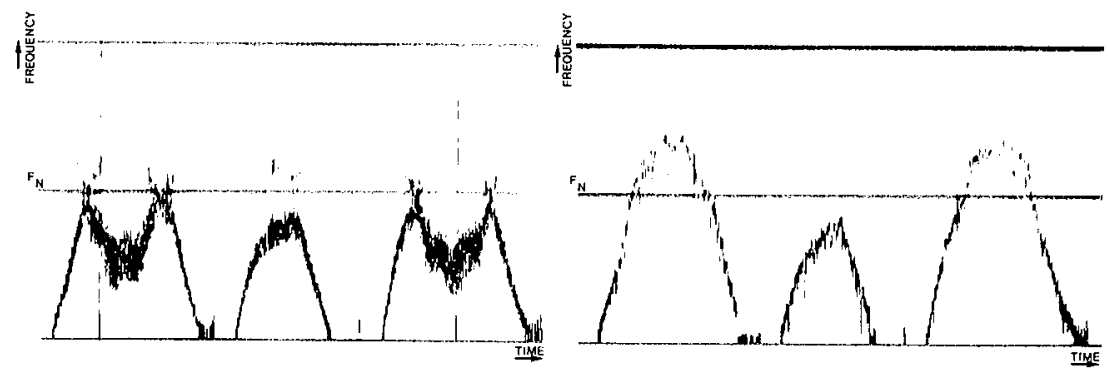

Fig. 6.22: Frequency aliasing shows up in the non-directional sonogram of a string target (fig. 6.22a). Implementation of a signal synthesizer utilizing the detected average frequency (after correction) and signal amplitude results in a sonogram as depicted in fig. $6.22 b$. 


\section{CLINICAL APPLICATIONS}

The applicability of multi-gate pulsed Doppler systems to visualize the velocity distribution along the cross-section of an artery has been demonstrated by several authors (Peronneau, 1974b; Anliker, 1976; Keller, 1976; Green, 1977; Brandestini, 1977; Stevenson, 1981; Bruins Slot, 1981; Hoeks, 1981; Reneman, 1981a; Reneman, 1981b). To avoid manipulation of the position of the sample volumes they should cover a range substantially greater than the diameter of the investigated arteries. Detail in the assessed velocity distribution can only be obtained if the dimensions of the sample volumes are small with respect to the diameter of the arteries (high resolution systems). Application of multi-gate pulsed Doppler systems with a low emission frequency (in the order of $3 \mathrm{MHz}$ ) and, therefore, large sample volumes (low resolution systems) will result in smooth velocity profiles which appear to be rather parabolic (Anliker, 1976; Keller, 1976). Low resolution systems rely heavily on the spectral content of the Doppler signal originating from a selected sample volume rather the assessed velocity distribution. The spectral content reflects thus the disturbed velocity distribution within the sample volume and can be revealed by spectrum analysis (Blackshear, 1979). Decreasing the size of the sample volume, which is possible if a higher emission frequency is selected, reveals the blunt nature of the pulsatile velocity distribution in peripheral arteries (Reneman, 1981b). High resolution systems show a lower signal-to-noise ratio and its transcutaneous application is limited to the investigation of superficial arteries like carotid and femoral arteries or, for example, pediatric cardiology.

Only a few authors (Reneman, 198la; Reneman, 198lb; Bruins Slot, 1981) reported about the use of a high resolution system in the evaluation of the peripheral circulation. Although the system used by Bruins slot (Bruins slot, 1981), which was the first generation of the system developed in Mastricht (Hoeks, 1981), had an axial resolution worse than $1 \mathrm{~mm}$, the number of 
126

gates indicating the presence of blood flow in the femoral arteries provided an estimation of the arterial diameter that correlated highly with the angiographic findings. Waveform analysis (pulsatility index, transit time measurements) executed on the velocity tracing recorded in the center of the blood stream with that multi-gate system and with a conventional $\mathrm{CW}$-system did not provide conclusive information about what system should be preferred to diagnose atherosclerotic lesions in the common and superficial femoral arteries (Bruins slot, 1981).

In other reports (Reneman, 1981a; Reneman, 1981b) a preliminary evaluation has been given of the time-dependent velocity profiles assessed with the system as described in chapter 5. It was demonstrated that the anatomical structure (diameter, bifurcation) and/or the presence of a stenosis affects the time dependent shape of the velocity distribution along the crosssection of the carotid arteries. To obtain a profound insight in the behaviour of velocity profiles as recorded in the carotid arteries with this improved system, a survey study was recently initiated. For this purpose 100 male volunteers, ranging in age from 20 to 70 years, were selected randomly from the population registers. The volunteers (pooled in age categories of 10 years) had normal blood pressures (diastolic blood pressure below $90 \mathrm{~mm} \mathrm{Hg}$ ) and they did not show any evidence of an atherosclerotic disease.The Doppler examination consisted of the construction of a global ultrasonographic image (section $5.8)$ to determine site and orientation of the carotid bifurcation, whereafter velocity profiles were recorded at specific points in the common, internal and external carotid arteries. Although up to now some 100 volunteers and patients have been examined the study of the reference group is not yet completed. A separate report will deal with the final results. The examples given below about the time-dependent behaviour of velocity profiles and the change in artery diameter during the cardiac cycle are intended to illustrate the applicability and limitation of a high-resolution multi-gate pulsed Doppler system. 
Under physiological conditions the velocity waveforms recorded simultaneously at $0.5 \mathrm{~mm}$ intervals along the ultrasound beam within the artery with a high-resolution system are rather similar. Fig. 7.1 shows the velocity signals assessed trans-

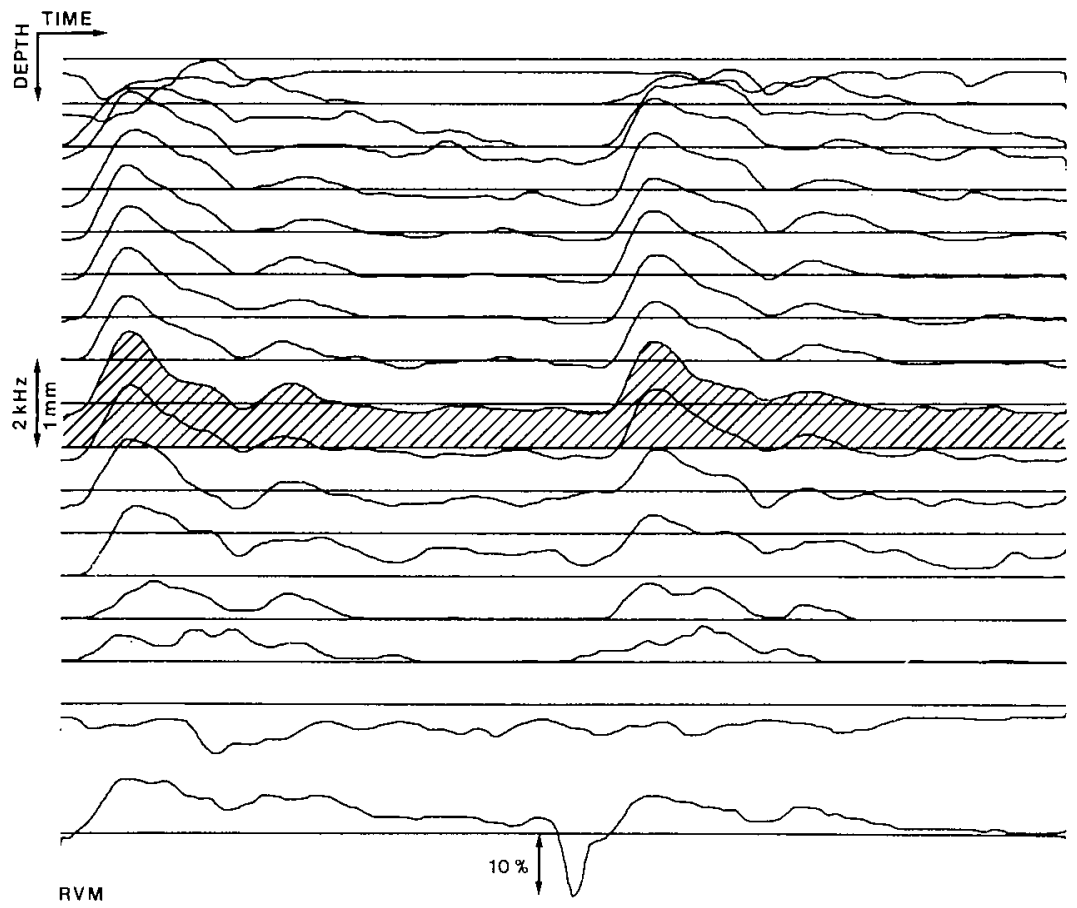

Fig. 7.1 : The velocity as continuous function of time as recorded at range intervals of $0.5 \mathrm{~mm}$ in the common carotid artery of a young healthy volunteer. To indicate the velocity tracing with respect to its zero line the area under the velocity recorded center stream is shaded. The bottom tracing represents the relative change in artery diameter.

cutaneously in the common carotid artery of a young healthy adult. In the upper tracing a steady negative velocity is observed, indicating that this flow signal is originating from the adjacent jugular vein. The center stream average Doppler frequency (based on the density of zero crossings) is reaching a maximum value in the order of $2.6 \mathrm{kHz}$ while in the diastole the average Doppler frequency decreases to $1 \mathrm{kHz}$. For clarity the area under the velocity tracing is shaded. Without this 
128

shading the corresponding zero-level can only be distinguished if the adjacent tracings are taken into account. The same velocity pattern can be observed in a large number of sample volumes confirming the blunt nature of the velocity profile in this artery. The bottom tracing shows the relative change in vessel diameter as function of time recorded simultaneously with the velocity signals. The negative deflection indicates the occurrence of the top of the R-wave of the ECG and serves as calibration level ( $10 \%)$ for the relative vessel wall movements (RVM). It can be observed that the maximum change in artery diameter in this subject is in the order of $10 \%$. In the first displayed heart cycle the signal does not completely return to zero. This is typical for most of the recordings that have been made up to now. One explanation for this phenomenon is a varying diastolic pressure causing a change in diastolic vessel diameter. Other factors contributing to an irregular waveform are system and/or operator oriented. Only Doppler signals with frequencies in the range from 5 to $300 \mathrm{~Hz}$ are taken into account to detect a change in the position of the vessel wall with respect to the probe. Slow variations as may occur in the diastolic phase of the cardiac cycle will therefore not be observed. Because the system automatically corrects for movements of the probe with respect to the vessel (see section 5.7) probe movements along the axis of the ultrasound beam will not affect the result. However, lateral movements of the ultrasound beam wih respect to the vessel will result in a change of the observed vessel diameter and will cause an irregular RVM-waveform. In practice it may be difficult to recognize the source of the error. It is assumed that the RVM-waveform reflects the relative change in artery diameter as function of time if the shape of the RVM-waveform is reproduced in subsequent heart cycles.

The feature to detect transcutaneously the relative change in the diameter of the common carotid artery is evaluated by subjecting the male volunteers to test measurements. In the age category of 20 to 30 years the average peak value of the RVM- 
waveform appeared to be $10.28 \quad(n=10$, standard deviation 1.148$)$. In the age category of 40 to 50 years the peak value decreased to $6.18(\mathrm{n}=10$, standard deviation $0.74 \%)$. The initial measurements in the other age categories indicate a sharp decline in the peak value in the age category of 30 to 40 years and a stabilization at a value around $6 z$ for subjects above 50 years of age. Only if the measurements of the RVM-waveform in the other age categories are completed and the effect of lesions on the RVM-waveform is estabiished it can be concluded whether the RVM-waveform can serve as a diagnostic tool in the diagnosis of vessel wall abnormalities.

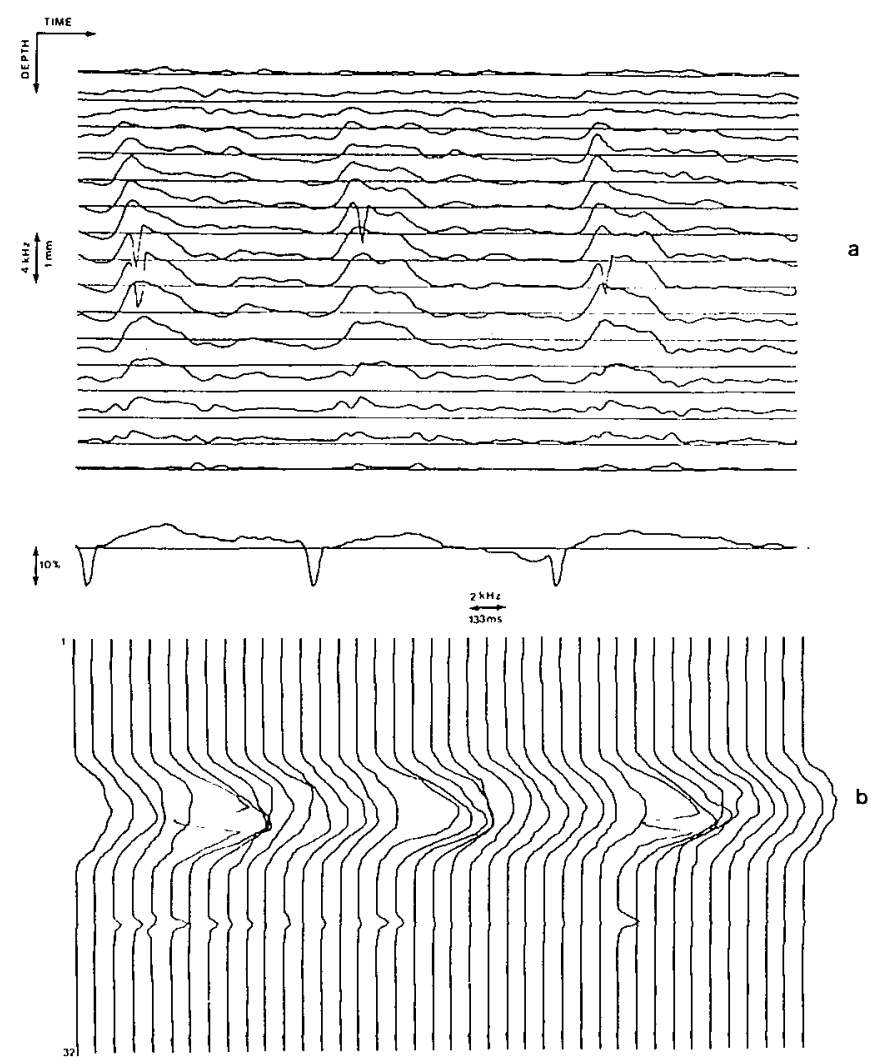

Fig. 7.2 : Velocity pattern as recorded distal to a stenosis in the common carotid artery of an asymptomatic volunteer. Both the velocity (7.2a) and profile (7.2b) mode exhibit the asymmetric velocity distribution along the cross-section of the artery, indicating a stenosis located in the anterior wall. 
Eig. 7.2 demonstrates the effect of a stenosis in the common carotid artery on the temporal and spatial behaviour of the velocities recorded distal to the stenosis. Since the stenosis was asymptomatic (discovered by chance with a volunteer in the age category of 50 to 60 years) angiography was not performed and data about the exact position of the stenosis and the extent of narrowing are not available. The presence of the stenosis was established by a conventional pulsed Doppler system with two-dimensional B-mode imaging showing locally a spectral broadening of the Doppler signal (Blackshear, 1979). A rather steady forward flow is observed near the proximal wall (fig. 7.2a) while near the anterior wall the velocity is pulsatile. From anterior to posterior wall the velocity waveform changes gradually in shape. The peak average Doppler frequency is beyond $4.75 \mathrm{kHz}$. The sudden negative deflections appearing in mid-systole are due to the selected output range of the Doppler system and should not be attributed to frequency aliasing (then a reversal in flow direction should occur). Because the anticipated average Doppler frequency will generally be substantially lower than the maximum Doppler frequency (see section $6.2)$ the output range of the system is limited to $4.75 \mathrm{kHz}$ by discarding the most significant bit. This does not affect the detection of the average Doppler frequency. The sudden negative deflections are, therefore, due to digital overflow, and average Doppler frequencies in the range of $P R E / 4$ to $P R E / 2$ will appear in the range of 0 to $\mathrm{PRF} / 4$. The recorded RVM-waveform appears to be rather smooth. The rapid change in artery diameter in early systole as present in $\mathrm{fig} .7 .1$ is not present in fig. 7.2a. Moreover, the peak value is reduced to approximately $5 \%$ indicating less elastic vessel walls and/or a change in the pulsatile behaviour of the local blood pressure. Fig. $7.2 a$ emphasizes mainly the temporal characteristics of the velocity signals. The spatial behaviour appears more clearly in fig. $7.2 b$ where the velocity distribution along the ultrasound beam down to a depth of $32 \mathrm{~mm}$ is presented at distinct time intervals. The sudden deflections are again due to overflow of the velocity output. Because the velocity profiles are only dis- 
played at time intervals of $66 \mathrm{~ms}$ not all the overflow situations shown in fig. $7.2 \mathrm{a}$ are present in $\mathrm{fig} .7 .2 \mathrm{~b}$. It is apparent that the velocity profile is asymmetric while the shape changes during the cardiac cycle. In early systole the velocity profile tends to move to the posterior wall and in late systole in the opposite direction. In the diastolic phase the observed profile is rather symmetric. This temporal behaviour of the velocity profile indicates the presence of an anterior wall stenosis proximal to the site of measurement. A posterior wall stenosis shows the opposite effect as demonstrated in fig. 7.3. Again the stenosis was discovered by chance (age category volunteer 50 to 60 years) and diagnosed on the basis of local spectral broadening (Blackshear, 1979). The exact site and the

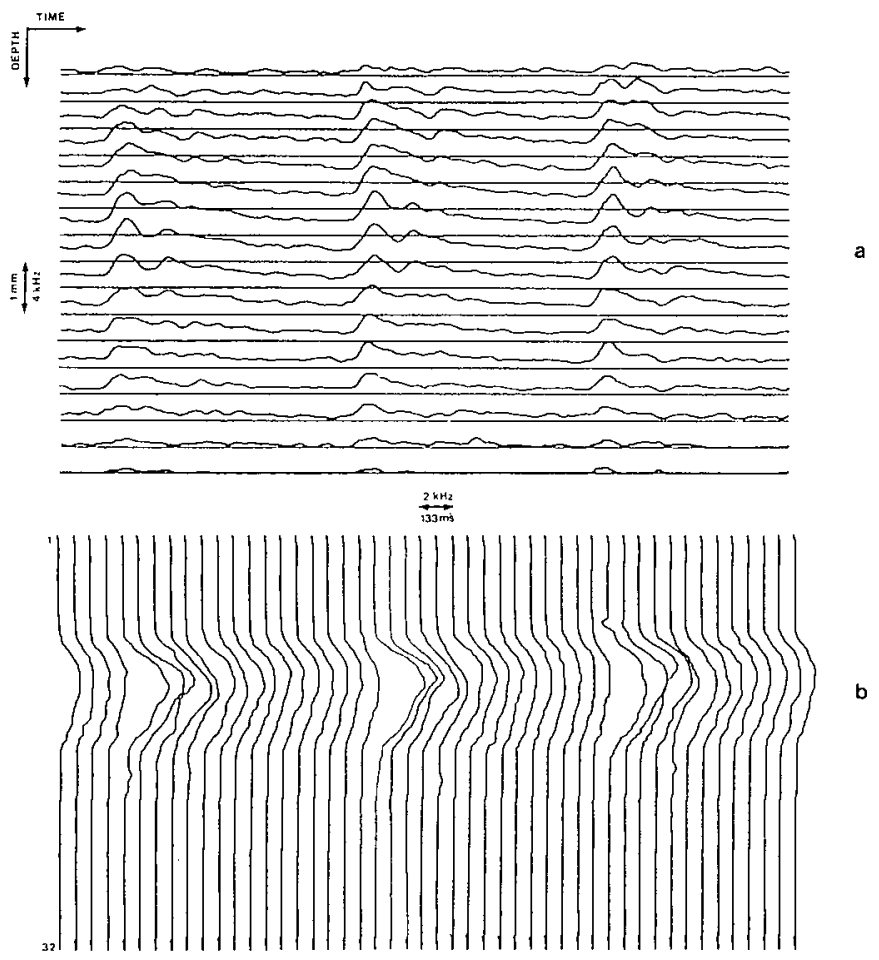

Fig. 7.3 : Velocity pattern as recorded distal to a posterior wall stenosis in the common carotid artery. This stenosis affects the spatial behaviour of the velocity patterm in an opposite way as an anterior wall stenosis (cf. fig. 7.2). 
spectral broadening (Blackshear, 1979). The exact site and the extent of the stenosis was not substantiated by angiographic findings (no symptoms). The tracings in fig. 7.3b, recorded distal to the stenosis located in the common carotid artery, show that in early systole the peak velocity is observed near the anterior wall while in late systole the peak shifts towards the posterior wall. In late diastole the velocity profile regains its symmetry. The way the stenosis affects the temporal behaviour of the velocity at distinct sites within this artery is demonstrated in $\mathrm{fig} .7 .3 \mathrm{a}$.
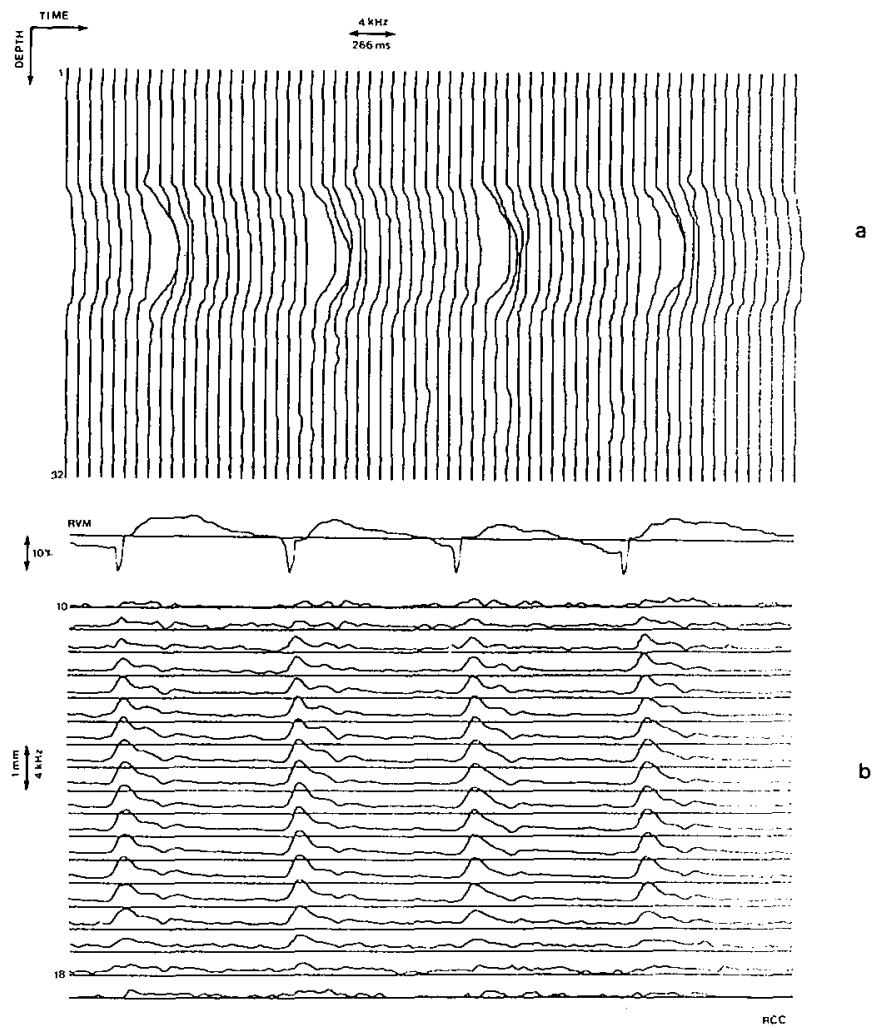

Fig. 7.4 : Velocity recordings made in the right common carotid artery $3 \mathrm{~cm}$ proximal to the bifurcation of a patient with a $30 \%$ stenosis located at the orifice of the left intermal carotid artery (see fig. 7.5). 
The differences in temporal and spatial behaviour of velocities between normal and obstructed flow can be better appreciated if the recorded velocities on both the unaffected ( $f$ ig. 7.4) and affected (fig. 7.5) side are presented simultaneously. The recordings were made in the right (fig. 7.4) and left common carotid artery of a patient in the age category of 60 to 70 years at approximately the same site with respect to the carotid bifurcation ( $3 \mathrm{~cm}$ proximal to $i t$ ). Angiography showed a local narrowing estimated at $30 \%$ due to a stenosis at the posterior site of the left carotid bifurcation. Fig. 7.4 shows a normal velocity behaviour for the age group of the subject. The
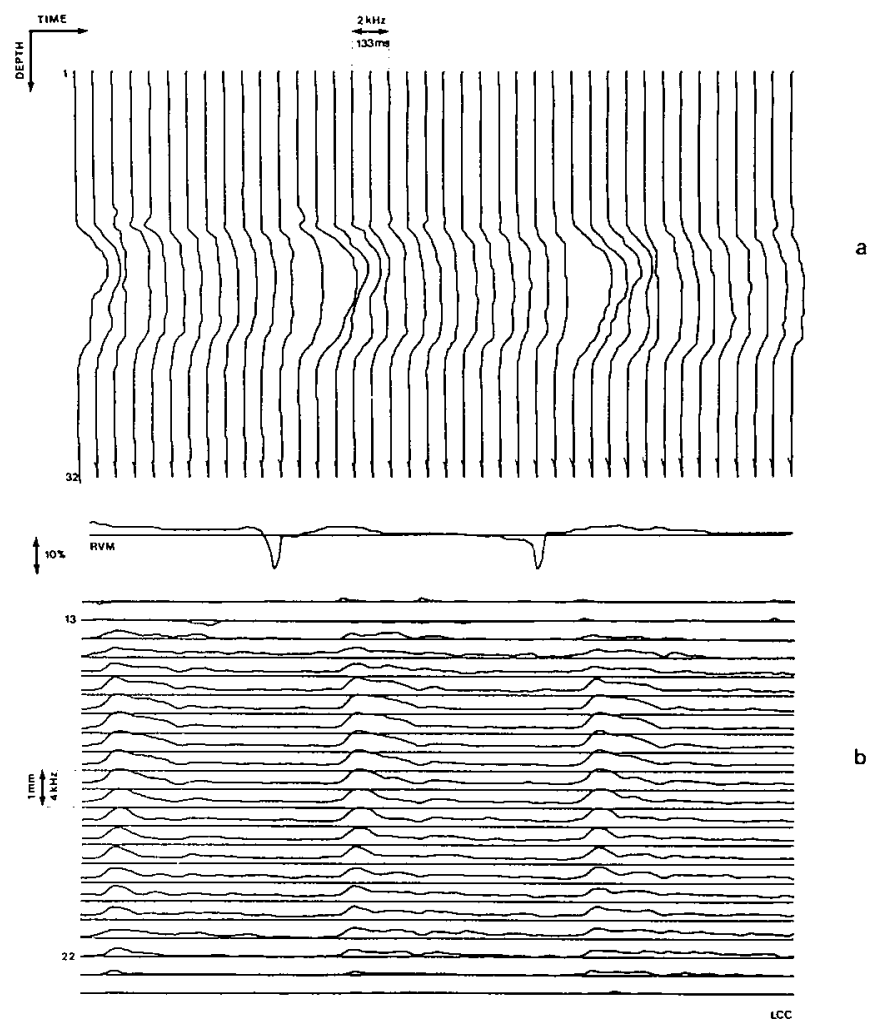

Fig. 7.5 : Velocity pattem recorded in the left common carotid artery $3 \mathrm{~cm}$ proximal to the bifurcation. The subject had a stenose at the orifice of the left internal carotid artery causing $30 \%$ reduction in diameter. The recordings made at the unaffected site of this patient were given in fig. 7.4. 
134

RVM-waveform is rather smooth and its peak value is in the order of 5\%. The velocity recordings at the diseased site (fig. 7.5) are quite different. Although the recordings are made proximal to the hemodynamic obstruction the temporal behaviour of the velocities (fig. 7.5a) near the posterior wall (diseased site) is quite different from the velocities recorded near the anterior wall. The asymmetric velocities along the cross-section of the left common carotid artery is confirmed by the velocity profiles as shown in fig. $7.5 \mathrm{~b}$. The peak value of the relative change in vessel diameter waveform is very low (in the order of 3\%) indicating (atherosclerotic) vessel walls and/or less pulsatile blood pressure at the site of recording. The last possibility is not likely since the subject had a normal blood pressure, the recording is made proximal to the stenosis and the angiogram did not show atherosclerotic disease proximal to the site of the recording. Fig. 7.5 indicates that a change in hemodynamical behaviour proximal to the site of the lesion can be detected with a high resolution multi-gate pulsed Doppler system.

In the applications as discussed above the registrations were made with a multi-gate pulsed Doppler system with an average frequency detector based on the density of zero crossings. In section 6.4 it was shown that at a high average frequency with respect to the $\mathrm{PRF}$, the zero crossing detector would cause a substantial error in the estimated average signal frequency, especially for large signal bandwidths. It is complicated to verify the effect of processing errors on the output of the Erequency detector because these errors are not only due to the sampled format of the Doppler signals but probably also due to frequency aliasing as well. Fig. 7.6 shows the velocity and profile tracings as function of time and depth in the common carotid artery of a young healthy male volunteer. The pulse repetition frequency was set at $9.5 \mathrm{kHz}$. The average Doppler frequency reaches a maximum in the order of $3.75 \mathrm{kHz}$. At these elevated flow rates the underestimation of the average frequency (being a function of the average frequency) will cause a 
distortion in the velocity tracings (see section 6.4). Since there was no evidence for vessel wall abnormalities the smooth top in the velocity tracing should be attributed to processing errors. The saturation of the velocity detector also shows up in the velocity distribution across the vessel (velocity profile) which is extremely flat. As an alternative for a frequency detector based on the density of zero crossings a frequency detector based on the instantaneous frequency corrected for processing errors was proposed (see section 6). Fig. 7.7 shows the recordings made with the second system in the common carotid artery of the same volunteer under the same recording conditions (although the angle of observation may deviate). During peak systole maximum velocities in the same order of magnitude and even some what higher (up to $4 \mathrm{kHz}$ ) are observed, but the temporal behaviour is distinctly different. The velocity distribution across the vessel exhibits a smoother appearance as well (since a memory unit with slightly different
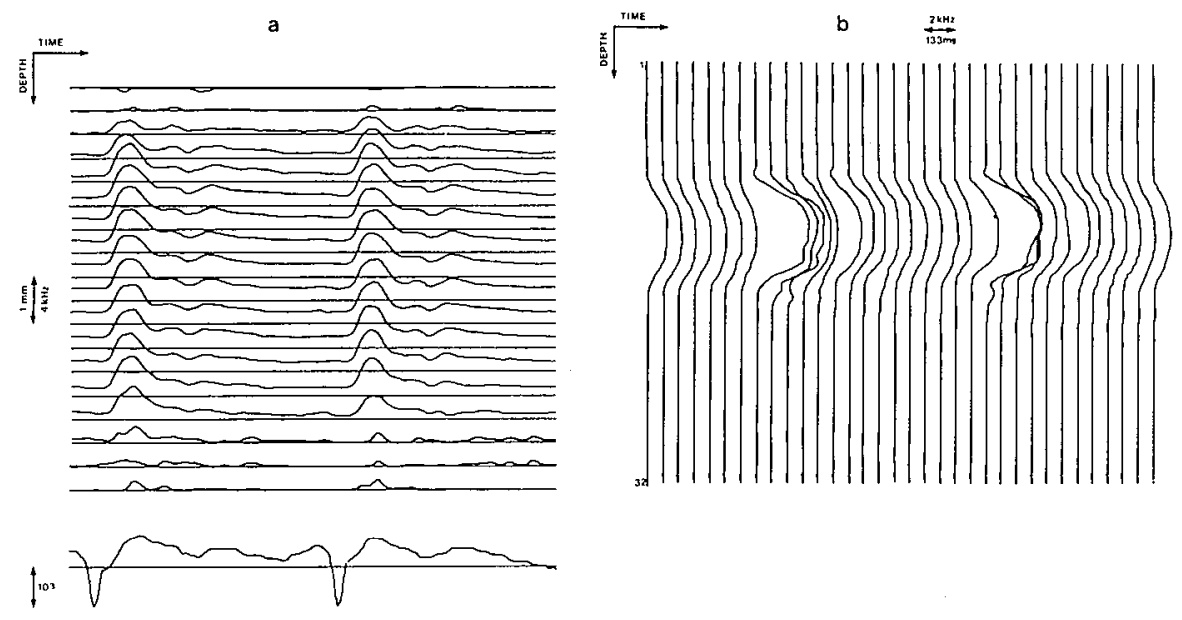

Fig. 7.6 : Velocity recordings made in the common carotid artery of a young healthy volunteer exhibiting elevated flow rates. The recording was made with a PRF of $9.5 \mathrm{kHz}$ while the peak velocities are in the order of $3.75 \mathrm{kHz}$. The rounded velocity tracings at peak systole (fig. 7.6a) indicate truncation due to the limited frequency range of the system. The saturation of the velocity detector causes an extremely flat profile (fig. 7.6b) at peak systole. 
characteristics is used the calibration level and time scale deviates with respect to examples given earlier). The system has yet to be evaluated in patients with disturbed velocity distributions.
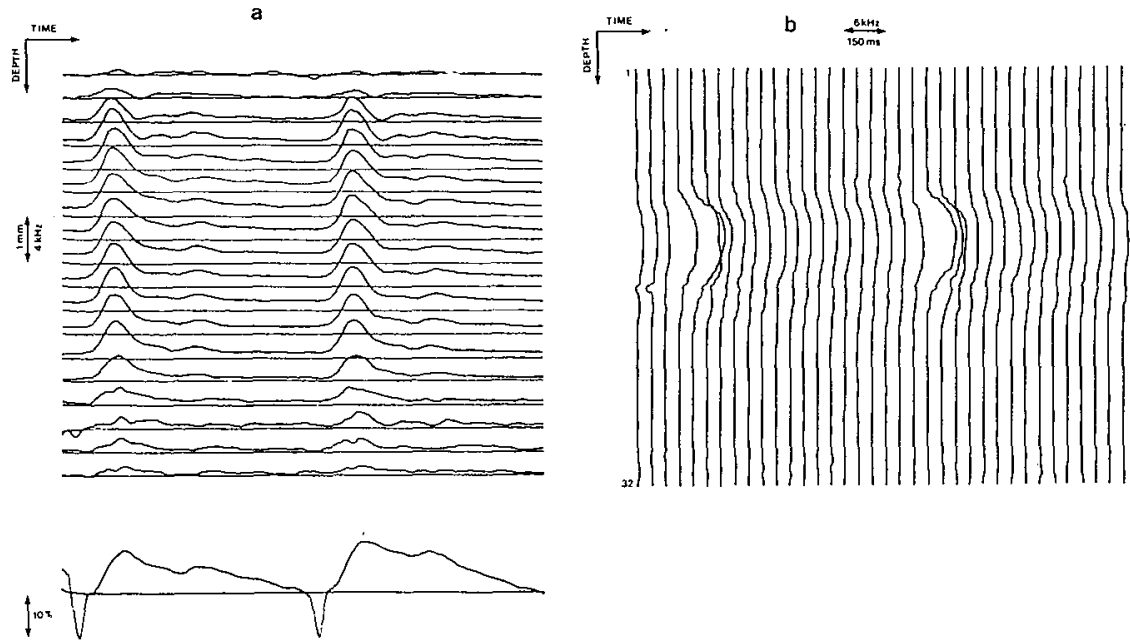

Fig. 7.7: Velocity pattern recorded at the same site of the same volunteer (see fig. 7.6) with a system equipped with a frequency detector based on the instantaneous frequency corrected for processing errors. A pulse repetition frequency of $9.5 \mathrm{kHz}$ was used. The systolic peaks (fig. 7.7a) are somewhat higher (which may be due to a change in the angle of observation) and sharper. The profile $(f i g .7 .7 b)$ has a smoother appearance. 
8. CONCLUSION

The application of pulsed Doppler systems sets requirements to the design of the system, and especially the dimension of the sample volume. A close collaboration between the clinician and the design engineer ensures a proper selection of the system parameters. If it is the only objective to reject Doppler signals originating from adjacent vessels and/or adjacent vessel walls one will opt for a mono-gate pulsed Doppler system employing a sample volume with a dimension slightly less than the diameter of the arteries of interest. A further reduction of the size of the sample volume will make it more complicated to position the sample volume and maintain it in a particular position with respect to the arterial walls. To reveal disturbed flow patterns further processing (spectral analysis, waveform analysis) is required. The analysis of the Doppler signal will become more delicate for smaller hemodynamic disturbances, since its contribution to the assessed Doppler signal will be minor. The disturbance can be singled out if a high resolution (small sample volume with respect to the diameter of the investigated artery) pulsed Doppler system is employed. With a small sample volume more sites have to be explored for adequate detection of flow disturbances. This will prolong the time of measurement. A combination with an echo system is required to get feedback about the site of measurement. Unfortunately, the echo system will affect the performance of the pulsed Doppler system. Since both systems should share the available pulse repetition frequency (PRF) simultaneous operation of both systems demanas for a reduction in the up-date rate of the echo image and a reduction in the Doppler frequency range of the Doppler system.

High resolution can be achieved if a relatively high emission frequency is selected and other system parameters (duration of emission, bandwidth and dimensions of the transducer as well as bandwidth of the receiver and the phasedetector) are dimensioned accordingly (see chapter 4). Scaling of system 
138

parameters as a consequence of a change in emission frequency is facilitated if the parameters are expressed with respect to the emission frequency or to the wavelength of the acoustic signal. To obtain a narrow ultrasound beam (small lateral dimension of the sample volume) over a long range the diameter of the transducer should be in the order of 20 wavelengths $(5 \mathrm{~mm}$ for an emission frequency of $6 \mathrm{MHz}$ ). Then the ultrasound beam starts to diverge at a range of $d^{2} / 4 \lambda=5 d$ (see section 2.2). The point of divergence will move along the axis of the ultrasound beam in relation to the selected emission frequency. For a slightly focussed transducer the diameter of the ultrasound beam at the focal point will be in the order of $4 \lambda$. From the point of the receiver the ultrasound beam appears more narrow because the focussing action occurs twice, reducing the apparent width of the ultrasound beam to about $3 \lambda$ (see section 5.9). The axial resolution should be in the same order or slightly less. This requires the emission of a pulse containing 4 periods. The limited bandwidth of transducer and preprocessing section will tend to elongate the pulse to the equivalence of 3 wavelengths, where the emit/receive action along the same line is taken into account. To accommodate a pulse containing 4 periods the bandwidth of the transducer and receiver should be in the order of $1 / 4$ of the employed emission frequency, while the bandwidth of the phase detector is half of the bandwidth of the receiver. If one maintains the above mentioned relations, the lateral and axial dimensions of the sample volume at the focal point in terms of wavelengths will be independent of the choice of emission frequency. The only effects are the signalto-noise ratio and the point of divergence of the ultrasound beam. Since now both the Doppler frequency and the point of divergence are linearly related to the emission frequency the maximum velocity that can be detected by a common pulsed Doppler system up to the point of divergence is constant.

As stated before the employment of a single channel pulsed Doppler system with a small sample volume with respect to the diameter of the artery under investigation requires elaborate 
exploration. The time needed for the examination can be reduced considerably if simultaneously the velocities along the crosssection are made available.This can be achieved with multi-gate pulsed Doppler systems. With these systems one is assured that the recordings are made under the same physiological conditions (pressure gradient, heart rate, respiration, condition vessel) facilitating evaluation of the assessed velocity signals. Detail in the velocity distribution can only be obtained if a large number of sample volumes with small dimensions are recorded simultanesouly. To construct a multi-gate pulsed Doppler with parallel processing (high-pass filtering and detection of average Doppler frequency) will evoke a lot of technical problems. Not only the processing channels for the sample volumes should be and remain identical but also electrical interference may occur, impairing the functioning of the instrument. Processing of the Doppler signals in a serial way by a single processing circuit ensures a minimum of tuning and maintenance. Identical processing of the signals is automatically taken care of. The required hardware is virtually independent of the number of sample volumes. Serial processing can be realized in analog form (Grandchamp, 1975; Nowicky, 1981) but this limits the performance and application of the system. Performing the required signal processing by digital circuitry allows a more versatile approach (see chapter 5) like the on-line display of the signal amplitude in different frequency ranges, the detection of vessel wall movements and the on-line display of the recorded velocity signals. Although digital processing offers distinct advantages over processing in analog form it has some drawbacks, which are mainly caused by the required processing rate. For a multi-gate pulsed Doppler system with a sample interval of $0.5 \mathrm{~mm}$ the in quadrature Doppler signals should be processed within $330 \mathrm{~ns}$. This limits the arithmetical operations that can be executed. A cascade of two digital filters of the first order is employed to suppress low frequency Doppler signals. This requires two additions and hardwired scaling. A digital filter of the second order would result in a more efficient rejection of unwanted Doppler signals but this requires 
140

multiplication by constants that are quite critical. A true digital multiplication would consume too much time and can, therefore, only be implemented if the required processing rate is considerably lower. In the proposed system the analog to digital conversion is incorporated in the digital filter. Analog to digital conversion as part of a digital filter of the second order would cause additional problems. To compensate for the transfer characteristic of the digital filter of the first order the cut-off frequency (see section 5.3) of the filter had to be selected somewhat higher then would be necessary for a filter of the second order. The above mentioned limitations are less significant if the sample volumes are larger (as in cardiology) because then the processing rate is accordingly lower.

Although the discussed system has been designed for peripheral examinations it can easily be converted into a system suited for cardiac applications because of its digital nature. This would involve the replacement of the internal oscillator (lower frequency) and transducer, adjustment of the bandwidth of receiver and phase detector and, if necessary, the selection of another cut-off frequency of the Doppler filter. The pulse repetition frequency and processing rate are scaled automatically with the oscillator frequency. As outlined before this adjustment will not affect the dimensions of the sample volume in terms of wavelengths. The ease with which the system parameters can be manipulated demonstrates another advantage of the digital approach.

In a system with serial data processing (either analog or digital) the signals are available in sampled form. The way the signal appears at the input of the frequency detector (continuous or sampled) affects the performance of the employed frequency detector (Angelsen, 1979). Although the signal is sampled adequately (no frequency aliasing) the average Doppler frequency will be underestimated, especially if the average frequency is high with respect to the sampling frequency and the bandwidth of the Doppler signal is large with respect to the 
average frequency (see section 6.4). Assuming a constant relation between bandwidth and average frequency the output of the conventional frequency detector based on the density of zero crossings per unit of time becomes less reliable at increasing average Doppler frequencies. Because of this error it may be doubted whether the output of the frequency detector truly reflects the average blood velocity in the sample volume. The situation becomes considerably worse if frequency aliasing occurs which is often not noticed. This would lead to the conclusion that a frequency detector based on the density of zero crossings only will function properly in systems with serial data processing if the average Doppler frequency remains below a frequency in the order of $\mathrm{PRF} / 4$ (dependent on the relative bandwidth of the Doppler signal and the error that can be tolerated) putting a severe constraint on the application of the system (only applicable under hemodynamically normal conditions).

As an alternative a frequency detector based on the instantaneous frequency is proposed. Since the signals are available in sampled form a true instantaneous frequency cannot be derived. This leads to a distribution of instantaneous frequencies of a Doppler signal with a non-zero average frequency contaminated by noise quite similar to the spectral distribution of an aliased signal. However, under the assumption that the bandwidth of the Doppler signal is limited to PRF/2 a correction can be made for processing and aliasing errors, provided that the initial average frequency of the signal is known. Under these conditions average Doppler frequencies exceeding the Nyquist-frequency can be detected correctly. Moreover, the proportional error in the output hardly depends on the average signal frequency and the signal-to-noise ratio. A conventional frequency detector (see section 3.5) exhibits a relative error which is strongly related to the signal-to-noise ratio. To obtain an error of less than 108 a signal-to-noise ratio of better than 10 is required. It has been demonstrated (see section 6.7 ) that the relative error in the output of a frequency 
142

detector based on the instantaneous frequency with correction for processing and aliasing errors is neglibly small, even for a signal-to-noise ratio of $0 \mathrm{~dB}$. The shape of the distribution of the instantaneous frequencies of a signal plus noise depends on the signal-to-noise ratio and the spectral distribution of the signal (it is assumed that the noise spectrum is uniform over the range from $-P R F / 2$ to $P R F / 2$ ). Decreasing the bandwidth of the Doppler signal without changing the signal-to-noise ratio, diminishes the width of the distribution of instantaneous frequencies. The variance in the estimator output is related to the shape of the distribution. A low signal-to-noise ratio and a large bandwidth of the Doppler signal causes a large variance in the estimator output. In the absence of a true Doppler signal (noise only) large fluctuations in the detected average frequency should be anticipated. Sample volumes located outside. the blood stream will then exhibit a very disordered velocity pattern. To suppress this behaviour the detected instantaneous frequency is set to zero if the envelope of the signal plus noise falls below a given level. Since the Doppler signal, because of its limited bandwidth, tends to suppress the noise contribution to the corrected instantaneous Erequencies the depth dependent gain can be set at a higher level for those regions where blood velocities are detected.

The application of this frequency detector makes it possible to detect velocity profiles under poor signal-to-noise conditions. Moreover, the demand for a high pulse repetition frequency to avoid frequency ambiguity (see section 4.1), is circumvented. The system is able to detect average Doppler frequencies exceeding the Nyquist frequency. To achieve this a correction algorithm is executed which takes in consideration the behaviour of the assessed velocities as function of time and of place. Up to now a real-time operation of a combined echo/Doppler system is impaired by the constraints put by the Doppler system the pulse repetition frequency (PRF). Since in the new approach the PRF for the Doppler system can be selected more freely a combined real-time echo/Doppler system, which 
functions properly also if high velocities are observed, becomes feasible.

The applicability of the high resolution multi-gate pulsed Doppler system with serial data processing to visualize in detail the velocity patterns proximal and distal to a stenosis has been demonstrated in chapter 7. The display of velocity patterns in either velocity or profile mode emphasizes the temporal and spatial behaviour, respectively. For this the combination with a memory unit that can store and display velocity distributions in scroll mode over the last 3 seconds of recording in essential. The storage module incorporated in the multi-gate system releaves the examiner from distracting tasks. If no recording is made of the relative change in vessel diameter during the cardiac cycle the only manipulations he has to perform are the selection of the sample volume for aural evaluation of the Doppler signal (by a footswitch) and the initiation the FREEZE-mode of the storage unit (also by a footswitch). The selection of the output mode and the data to be copied can be made afterwards (see section 5.10). Only the recording of the movements of the vessel walls involves an interactive procedure and requires an assistant, whose task it is to select the sample volumes coinciding with the anterior and posterior wall, based on the envelope distribution of the low frequency Doppler signals and the assessed velocity distribution. The peak value of the relative change in artery diameter during the cardiac cycle clearly depends on the age of the subject (see chapter 7). It has still to be investigated to which extent the change in diameter can be used as an additional tool in the diagnosis of vessel wall abnormalities because a number of factors contribute to a change in shape of the waveform reflecting the relative vessel wall movements. A reduction in its peak value can be due to a stiffening of the arterial walls, plaque formation, high blood pressure and/or a reduction in local fluctuations in blood pressure during the cardiac cycle. 
144

In this study no attempts have been made to qualify disordered flow patterns in an objective way. Although up to now 100 volunteers have been subjected to a bilateral examination of the carotid arteries objective rules to qualify the flow pattern other than its symmetry with respect to the vessel axis have not been developed yet. One reason for this is the unfamiliarity with the velocity pattern under pulsatile flow conditions and, henceforth, with the effect of an obstruction on the local velocity distribution. The situation becomes even more complicated if the obstruction is located at the lateral vessel wall with respect to the ultrasound beam rather than at the anterior or posterior wall. Most likely, this multi-gate system can contribute valuable information about the hemodynamic behaviour under physiological and pathophysiological conditions. 
A local narrowing of a blood vessel due to stenoses (partial occlusions) or plaque-formation affects the velocity distribution alongthe cross-section of the blood vessel in the vicinity of the constriction. The visualization of the disturbances in the time-dependent velocity profile requires a system, capable to detect non-invasively the velocity pattern. Pulsed Doppler systems render the possibility to assess transcutaneously the velocity of blood within a small sample volume. The position of the sample volume can be manipulated by the operator. A large sample volume is at the expense of the spatial resolution. On the other hand a small sample volume complicates the procedure to position the sample volume properly and to maintain it at the selected site. A multi-gate system, capable to detect simultaneously the velocity in a large number of adjacent sample volumes, circumvents the problems encountered in the localisation of the sample volume. Moreover, it provides the velocity distribution along the cross-section as an instantaneous function of time. This demands for sample volumes with small dimensions (requiring a large number of sample volumes to cover the cross-section). Reducing the spatial dimension of the sample volumes affects the accuracy with which the blood velocities, including its direction, can be detected. This thesis describes the development of a high resolution multi-gate pulsed Doppler system. Data-processing is accomplished in a serial way.

In chapter 2 a first orientation is given about Doppler systems based on ultrasound employing emission frequencies ranging from 2 to $10 \mathrm{MHz}$. Specific characteristics of two kinds of Doppler systems are discussed in chapter 3 (continuous wave systems) and in chapter 4 (pulsed systerns). Chapter 5 deals with the development of a high-resolution multi-gate pulsed Doppler system with serial data-processing. This system combines the capability to detect the velocity distribution along the ultreasound beam simultaneously with the detection of the relative change in artery diametar during a cardiac cycle. The 
146

spatial average of the velocity in a sample volume is detected by means of a conventional zero crossing detector. However, due to the specific nature of the Doppler signals and due to the way in which the signals are processed an error is introduced in the detected average velocity, while the maximal velocity, which can be estimated reliably, is limited. Chapter 6 deals with an alternative method to detect the average velocity within a sample volume, based on the time average of the instantaneous frequency of the Doppler signals. Computer simulations and in vitro experiments demonstrate that this approach circumvents some of the basic problems of pulsed Doppler systems (upper bound to the assessed velocities and the signal-to-noise ratio). In chapter 7 some clinical examples are presented to demonstrate the applicability of a high resolution multi-gate system. The assessment of the instantaneous velocity distribution over the cross-section of an artery simultaneous with the detection of the relative change in the diameter of the artery contributes to the understanding of the hemo-dynamic behaviour around a local constriction of a blood vessel. 


\section{SAMENVATTING}

Een lokale vernauwing (stenose, plaque) in een bloedvat beinvloedt de snelheidsverdeling over le jwarsdoorsnede voor, in en na de vernauwing. Om deze verstoringen in het snelheidsprofiel zichtbaar te kunnen maken heeft men een systeem nodig dat door de intakte bloedvatwand heen het (tijd-) afhankelijke snelheidspatroon kan registreren. Gepulste Doppler systemen bieden de mogelijkheid om door de intakte huid heen de bloedsnelheid te meten in een klein meetvolume warvan de positie binnen een bepala gebied gemanipuleerd kan worken. Is het meetvolume groot dan verliest men plaatsresolutie (men weet niet meer welke snelheidsinformatie van welke plaats komt). Is het meetvolume daarentegen klein dan is het een probleem om hat meetvolume op een gewenste plaats in het bloedvat te krijgen en het daar te houden. Met een meer-kanaals gepulst Doppler systeem, dat in staat is om gelijktijdig de snelheid te meten in een groot aantal aangrenzende meetvolumes vermijat men deze problemen ten aanzien van le lokalisatie. Bovendien verkrijgt men de snelheidsverdeling over de dwarsdoorsnede van een bloedvat als functie van de hartcyclus. Voorwarde is wel dat de meetvolumes voldoende klein zijn (dit vereist een groot aantal meetvolumes) en dat dit niet ten koste gat van de nauwkeurigheid warmee men de snelheden, inclusief de richting, kan meten. In dit proefschrift worat de ontwikkeling van een meer-kanaals gepulst Dopplersysteem beschreven. De gegevens-verwerking vindt op een seriele manier plats (waardoor de "kanalen" hun betekenis verliezen) in tegenstelling tot eerder beschreven systemen warin de gegevens via aparte kanalen werjen verwarkt.

In hoofdstuk 2 wordt een eerste orientatie gegeven van Doppler systemen die gebaseer 3 zijn op ultrageluid ( 2 - 10 MHz), terwijl in hoofdstuk 3 en 4 dieper wordt ingegaan op de specifieke eigenschappen van twee types Doppler systemen (continuous wave in hoofdstuk 3 en gepulst in hoofdstuk 4). In hoofdstuk 5 wordt gedetailieerd ingegaan op het ontwikkelde gepulste systeem met seriele data-verwerking. Het systeem is in staat om 


\section{8}

tegelijkertijd de snelheidsverdeling langs de geluidsbundel en de relatieve veranderingen in de diameter van het bloedvat te detekteren. Voor de bepaling van de gemiddelde snelheid in een meetvolume worat gebruik gemaakt van een nulpuntenteller. Vanwege het specifieke karakter van de signalen en de manier warop deze verwerkt worden ontstat er niet alleen een fout in de gedetekteerde gemiddelde snelheid mar wordt ook de maximale snelheid die men betrouwbaar (inclusief richting) kan meten beperkt. In hoofdstuk 6 wordt ingegaan op een alternatieve methode om de gemiddelde snelheid in een meetvolume te bepalen, gebaseera op het tijayemiddelde van de instantane frequentie van het Dopplersignaal. Met behulp van computersimulaties en in vitro metingen wordt aangetoond dat deze benadering een oplossing biedt voor de fundamentele problemen van een gepulst Doppler systeem nI. de beperkte mogelijkheid om hoge snelheden te meten en de invloed van de signaal-ruis-verhouding op het resultat. In hoofdstuk $7 \mathrm{zijn}$ een aantal klinische voorbeelden gegeven van de toepassingsmogelijkheden van een meer-kanals gepulst Doppler systeem. Geconcludeerd kan worden dat de registratie van de instantane snelheidsverdeling over de dwarsdoorsnede van een bloedvat gecombineerd met de gelijktijdige detectie van vaatwandbewegingen een belangrijke bijarage levert tot een beter inzicht in het hemodynamische gedrag rondom lokale vernauwingen in bloedvaten. 


\section{REFERENCES}

Ackroyd M.H. (1973): Digital filters.

In: Computers and Medicine series. Ed.: D.W. Hill. Publ.: Butterworth \& Co., London.

Angelsen B.A.J. (1975): Transcutaneous measurement of aortic blood velocity by ultrasound. A theoretical and experimental approach. Dissertation, report 75-78-W, University of Trondheim, Norway.

Angelsen B.A.J., Kxistoffersen K. (1979): On ultrasonic MTI measurement of velocity profiles in blood flow.

IEEE Trans. on Biomedical Engineering, vol. BME-26, nr. 12, 665-671.

Angelsen B.A.J. (1980a): A theoretical study of the scattering of ultrasound from blood.

IEEE Trans. on Biomedical Engineering, vol. BME-27, $\mathrm{nr} .2,61-67$.

Angelsen B.A.J. ( $1980 \mathrm{~b})$ : Spectral estimation of a narrow-band gaussian process from the distribution of the distance between adjacent zeros.

IEEE Trans. on Biomedical Engineexing, vol. BME-27, nr. 2, 108-110.

Anliker M. (1976): The instantaneous velocity profile in large arteries as a diagnostic indicator of abnormalities of the vessel wall surface.

In: Blood vessels, Ed.: S. Effert, J.D. Meyer-Erkelenz, Publ.: Springer Verlag, Berlin, 97-103.

Arndt J.O., Klauske J., Mersch F. (1968): The diameter of the intact carotid artery in man and its change with pulse pressure.

Pfluegers Archiv, 301, 230-240.

Arts (1972): On the instantaneous measurement of blood flow by ultrasonic means.

Med. \& Bio. Engng., vol. 10, 23-34.

Atkinson P. (1976): A fundamental interpretation of ultrasonic Doppler velocimeters.

Ultrasound in Med. \& Biol., vol. 2, 107-111.

Bakex D.W., Yates W.G. (1973): Technique for studying the sample volume of ultrasonic Doppler devices.

Med. and Biol. Engng., vol. 11, 766-770.

Barnes R.W., Toole J.F., MCKinney W.H. (1977): An ultrasound digital moving target indicator system for diagnostic use.

IEEE Trans. on Sonics and Ultrasonics, vol. SU-24, nr. 6, 350-354.

Baskett J.J., Beasley M.G., Murphy G.J., Hyams D.E., Gosling R.G. (1977): Screening for carotid junction disease by spectral analysis of Doppler signals.

Cardiovascular Research, vol. 11, nr. 2, 147-155.

Berry S.M., O'Donnell J.A., Hobson R.W. (1980): Capabilities and limitations of pulsed Dopplex sonography in carotid imaging.

J. Clin. Ultrasound, vol. 8, 405-412. 
Blackman R.B., Tukey J.W. (1959): The measurement of power spectra. Publ. Dover Publications, New York.

Blackshear W.M., Phillips D.J., Thiele B.L., Hirsch J.H., Chikos P.M., Marinelli M.R., Ward K.J. (1979): Detection of carotid occlusive disease by ultrasonic imaging and pulsed Doppler spectrum analysis. Surgery, vol. 86, nx. 5, 698-706.

Blackshear W.M., Phillips D.J., Chikos P.M., Harley J.D., Thiele B.L., Strandness D.E. (1980): Carotid artery velocity patterns in normal and stenotic vessels.

stroke, vol. 11, nr. 1, 67-71.

Bournat J.P., Peronneau P.A., Herment A. (1977): Yes-no ultrasonic Dopplex method of detection for vascular imaging.

Ultrasound in Medicine and Biology, vol. 3, 105-115.

Brandestini M. (1976): Die Signalverarbeitung in perkutanen Ultraschall Doppler Blutflussgeraeten.

Thesis E.T.H.-Zuerich, Suisse.

Brandestini M. (1978a): Topoflow - a digital full range Doppler velocity meter.

IEEE Trans, on Sonics and Ultrasonics, vol. SU-25, 287-293.

Brandestini M., Forster F.K. (1978b): Blood flow imaging using a discretetime frequency meter.

Ultrasonics Symp. Proc. IEEE cat. nr. 78, CH 1344-1SU, 348-352.

Brandestini M.A., Eyer M.A., Stevenson J.G. (1979): M/Q mode echocardiography - the synthesis of conventional echo with digital multigate Doppler.

In: Echocardiology. Ed.: C.T.Lancée. Martinus Nijhoff Publ. The Hague, 441-446.

Bruins slot H. (1981): Doppler studies in the femoro-popliteal pathway. Dissertation, University of Iimburg, Maastricht, the Netherlands.

Burckhardt C.B. (1981): Comparison between spectrum and time interval histogram of ultrasound Doppler signals.

Ultrasound in Med. \& Biol., vol. 7, 79-82.

Cathignol D.J., Fourcade C., Chapilon J.-Y. (1980): Transcutaneous blood flow measurements using pseudo-random wise Doppler system.

IEEE Trans, on Biomedical Engineering, vol. BME-27, nr. 1, 30-36.

Davenport W.B., Root W.I. (1958): An introduction to the theory of random signals and noise.

Publ.: McGraw-Hill, New York.

Day T.K., Fish P.J., Kakkar V.V. (1976): Detection of deep vein thrombosis by Doppler angiography.

Bxitish Medical Journal, vol. 1, 618-620. 
Eyer M.K., Brandestini M.A., Phillips D.J., Baker D.W. (1981): Color digital echo/Doppler image presentation.

Ultrasound in Med. \& Biol., vol. 7, 21-31.

Farrar D.J., Green H.D., Peterson D.W. (1979): Non-invasively and invasely measured pulsatile haemodynamics with graded arterial stenosis.

Cardiovascular Research, vol. 13, 45-57.

Fish P. (1973): Visualizing blood vessels by ultrasound.

In: Blood flow measurements. Ed.: C.Roberts. Publ.: Sector Publ. Ltd., London.

Fish P.J., Wilson I.M., Holt B., Walters D. (1978): Multichannel pulsed Doppler imaging: Measurement accuracy and beam/vessel angle estimation. In: Ultrasound in Medicine. Vol.4. Ed.: D. White, E.Lyons. Publ.: Plenum Press, New York, 359-362.

Forster F.K. (1977): The applications and limitations of Doppler spectral broadening measurements for the detection of cardiovascular disorders. Ultrasound in Med., Ed.: D. White, R. Brown, Publ.: Plenum Press, vol. $3 \mathrm{~B}, 1223-1226$.

Forster F.K., Baker D.W. (1978): Quantitative flow measurement utilizirig a time interval histogram of Doppler shifted ultrasound.

In: Ultrasound in Medicine. Vol. 4. Ed.: D. White, E.Lyons. Publ.: Plenum Press, New York, 349-353.

Gabor D. (1946): Theory of communications. Journal of IEE, nr. 93, 429-457.

Gerzberg I, Meindl J.D. (1980): Power-spectrum centroid detection for Doppler systems applications.

Ultrasonic imaging, vol. 2, 232-261.

Gill R.W., Meindl J.D. (1973): Optimal system design of the pulsed ultrasonic blood flowmeter.

IEEE Ultxasonics Symposium (nov. 1973), 88-93.

Gill R.W. (1979): Performance of the mean frequency Doppler modulator. Ultrasound in Med. \& Biol., vol. 5, 237-247.

Grandchamp P.-A. (1975): A novel pulsed directional Doppler velocimeter; The phase detection principle.

In: Proc. of the 2nd Eur. Congress on Ultrasonics in Medicine, Ed.: E. Kazner, Publ.: Exc. Medica, Amsterdan, 122-132.

Green P.S., Taenzer J.C., Ramsey S.D., Holzemer J.F., Suarez J.R., Marich K.W., Evans T.C., Sandok B.A., Greenleaf J.F. (1977): A real-time ultrasonic imaging system for carotid arteriography.

Ultrasound in Medicine and Biology, vol. 3, 129-142.

Hartley C.J. (1981): Resolution of frequency aliases in ultrasonic pulsed Doppler velocimeters.

IEEE Trans. on Sonics and Ultrasonics, vol. SU-28, nr. 2, 69-75. 
Haskway T., Raines J; (1980): Real-time ultrasonic imaging of the peripheral arteries; technique, normal anatomy and pathology.

Cardiovascular Disease, Bulletin of the Texas Heart Institute, vol. 7, nr. 3, 257-265.

Hoeks A.P.G., Reneman R.S., Ruissen C.J., Smeets F.A.M. (1979): Possibilities and limitations of pulsed Dopplex systems.

In: Echocardiology, Ed.: C.T. Lancée, Pụb1.: M. Nijhoff, The Hague, 413-419.

Hoeks A.P.G., Reneman R.S., Peronneau P.A. (1981): A multi-gate pulsed Doppler sytem with serial data processing.

IEEE Trans. on Sonics \& Ultrasonics, vol. SU-28, nr. 4, 242-247.

Hokanson D.E., Mozersky D.J., Summer D.S., Strandness D.E. (1972): A phase locked echo-tracking system for recording arterial diameter changes in vivo.

J. of Appl. Physiology, vol. 32, 728-733.

Hutchison K.J., Oberle K., Scott J.A. (1981): A comparison of Doppler ultrasonic waveforms processed by zero-crossing and spectrographic techniques in the diagnosis of peripheral arterial disease. Angiology, vol. 32, nr. 4, 277-289.

Jerri A.J. (1977): The shannon sampling theorem - its various extension and applications: a tutorial review.

Proc. of the IEEE, vol. 65, nr. 11, 1565-1596.

Jorgensen J.E., Campau D.N., Baker D.W. (1973): Physical characteristics and mathematical modeliing of the pulsed ultrasonic flowmeter. Med. and Biological Engineering, 404-421.

Keller H.M., Meier W.E., Anliker M., Kumpe D.A. (1976): Non-invasive measurement of velocity profiles and blood flow in the common carotid artery by pulsed Doppler ultrasound.

Stroke, vol. 7 , nr. 4, 370-377.

Khalifa A.M.A., Giddens, D.P. (1981): Characterization and evolution of poststenotic flow disturbances.

J. Biomechanics, vol. 14, nr. 5, 279-296.

Kossoff G. (1966): The effects of backing and matching on the performance of piezo-electric ceramic transducers.

IEEE Trans. on Sonics \& Ultrasonics, vol. SU-13, nr. 1, 20-30.

Lewis R.L., Beasly M.G., Hyams D.E., Gosling R.G. (1978): Imagining the carotid bifurcation using continuous wave Doppler shift ultrasound and spectral analysis.

Stroke, vol. 9, nr. 5, 465-471.

Lunt M.J. (1975): Accuracy and limitations of the ultrasonic Doppler blood velocimeter and zero-crossing detector.

Ultrasound in Med. \& Biol., vol. 2, 1-10. 
Lusby R.J., Woodcock J.P., Skidmore R., Jeans W.D., Hope D.T., Blaird R.N. (1981): Carotid artery disease: a prospective evaluation of pulsed Doppler imaging.

Ultrasound in Med. and Biol., vol.7, nr. 4, 365-370.

Mandel L. (1974): Interpretation of instantaneous frequencies. Am. J. of Physics, vol. $42,840-846$.

McDonald D.A. (1974): Blood flow in arteries. Camelot Press Lta., Southampton.

McLeod F.D. (1967): A directional Doppler flowmeter.

Digest of the 7 th International Conf. on Med. \& Biol. Engng, Stockholm, 196-217.

Mozersky D.J., Hokanson D.E., Sumuer D.S., Strandness D.E. (1972): Ultrasonic visualization of the arterial lumen. Surgery, vol. $72,253-259$.

Newhouse V.L., Furgason E.S., Chung T. Ho (1978): A technique for increasing the maximum range-velocity product of pulsed Doppler systems.

In: Ultrasound in Medicine. Vol. 4. Ed.: D.White, E.Lyons. Publ.: Plenum Press, New York, 355-358.

Newhouse V.L., Lecong P., Furgason E.S., Ho C.T. (1980): On increasing the range of pulsed Doppler systems for blood flow measurements. Ultrasound in Med. \& Biol., vol. 6, 233-237.

Olsen C.F. (1977): Doppler ultrasound: A technique for obtaining arterial wall motion parameters.

IEEE Trans. on Sonics and Ultrasonics, vol. SU-24, nr. 6, 354-358.

Oppenheim A.V., weinstein C.J. (1972): Effects of finite register length in digital filtering and the fast Fouxier transform.

Proc. IEEE, vol. 60, nr. 8, 957-967.

Peronneau P.A., Bournat J.-P., Bugnon A., Barbet A., Xhaard M. (1974a): Theoretical and practical aspects of pulsed Doppler flowmetry: real-time applications to the measure of instantaneous velocity profiles.

In: Cardiovascular applications of ultrasound, Ed.: R.S. Reneman, Publ.: North-Holland Publ. Co., Amsterdam-London, 66-84.

Peronneau P.A., Hinglais J.R., Xhaard M., Delouche P., Philippo J. (1974b): The effects of curvature and stenosis on pulsatile flow in vivo and in vitro.

In: Cardiovascular applications of ultrasound, Ed.: R.S. Reneman, Publ.: North-Holland Publ. Co., 203-215.

Reneman R.S., Hoeks A. (1977): Continuous wave and pulsed Doppler flowmeters - a general introduction.

In: Echocardiology, Ed.: N. Bom, Publ.: M. Nijhoff, The Hague, 189-205.

Reneman R.S., Spencer M.P. (1979a): Local Doppler audio spectra in normal and stenosed carotid arteries in man.

Ultrasound in Medicine and Biology, vol. 5, nr. 1, 1-11. 
Reneman R.S., Hoeks A., Spencer M.P. (1979b): Doppler ultrasound in the evaluation of the peripheral arterial circulation.

Angiology, vol. $30, \mathrm{nr} .8,526-538$.

Reneman R.S., Hoeks A., Ruissen C., Smeets F. (1981a): Pulsed Dopplex systems and cardiovascular disease.

In: Echocardiology, Ed.: H. Rijsterborgh, Publ.: M. Nijhoff, The Hague, $269-278$.

Reneman R.S. (1981b): What measurements are necessary for a comprehensive evaluation of the peripheral arterial circulation?

Cardiovascular Diseases. Bulletin of the Texas Heart Institute. Vol. 8, nr. 3, 435-454.

Rice S.O. (1944): Mathematical analysis of random noise. Bell system Tech. Journal, vol. 23, 282-332.

Rittgers S.E., Putney W.W., Barnes R.W. (1980): Real-time spectrum analysis and display of directional Doppler ultrasound blood velocity signals. IEEE Trans. on Biom. Engng., vol. BME-27, nr. 12, 723-728.

Roevros J.M.J.G. (1974): Analogue processing of C.W.-Doppler flowmeter signals to determine average fxequency shift momentaneously without the use of a wave analyzer.

In: Cardiovascular applications of ultrasound, Ed.: R.S. Reneman, Publ.: North-Holland Publ. Co., Amsterdam-London, 43-54.

Salz J., Stein S. (1974): Distribution of instantaneous frequency for signàl plus noise.

IEEE Trans. on Information theory, 272-274.

Sandmann W., Peronneau P., Schweins G., Bournat J., Hinglais J. (1978): Turbulenzmessung mit dem Doppler Ultraschallverfahren: eine neue Methode der Qualitatskontrolle in dex Arterienchirurgie.

In: Ultraschall-Dopplex-Diagnostiek in der Angiologie, Ed.: A. Kriesmann, A. Bollinger, Publ.: Thieme Verlag Stuttgart, 77-81.

Shung K.K., Sigelmann R.A., Reid J.M. (1976): Scattering of ultrasound by blood.

IEEE Trans. on Biomedical Engineering, vol. BME-23, nx. 6, 460-467.

Skidmore R., woodcock J.P. (1980): Physiological intexpretation of Doppler shift waveforms-I.

Ultrasound in Med. \& Biol., vol. $6,7-10$.

Skidmore R., Woodcock J.p. (1980): Physiological interpretation of Doppler shift waveforms-II.

ultrasound in Med. \& Biol., vol. 6, 219-225.

Skidmore R., Woodcock J.P., wells P.N.T., Bird D., Baird R.N. (1980): Physiological interpretation of Dopplex shift waveforms-III.

Ultrasound in Med. \& Biol., vol. 6, 227-231.

Skolnik M.I. (1962): Introduction to radar systems. MoGraw-Hill, New York. 
Stevenson J.G., Kawabori I., Brandestini M.A. (1981): A twentymonth experience comparing conventional pulsed Doppler echocardiography and colorcoded digital multigate Doppler for detection of atrioventricular valve regurgitation and its severity.

In: Echocardiology. Ed.: R.S.Rijsterborgh. M. Nijhoff Publ. The Hague, 399-407.

Uematsu, S. (1981): Determination of volume of arterial blood flow by an ultrasonic device.

J. Clin. Ultrasound, vol. 9, 209-216.

Wells P.N.T. (1969): Physical principles of ultrasonic diagnosis. Publ.: Academic Press, London and New York.

Wetterer E. (1968): Grundlagen dex Dynamik des Arterienpulses.

Publ.: Springer Verlag, Berlin, 238-241.

Wildi E., Knutti J.W., Allen H.V., Meindl J.D. (1980): Dynamics and limitations of blood/muscle interface detection using Doppler power returns.

IEEE Trans, on Biom. Engng., vol. BME-27, nr. 10, 565-573.

Wille s.0., Walloe I. (1981): Pulsatile pressure and flow in arterial stenoses simulated in a mathematical model.

J. Biomed. Engng., vol. 3, 17-24. 

NAWOORD

De projectgroep die in 1976 aan de Rijksuniversiteit Limburg begon met een onarrzoek naar de mogelijkheden van detectie van afwijkingen in bloedvaten met behulp van ultrageluid bestond voor het grootste gedeelte uit personen die niet vertrouwd waren met de mogelijkheden en problemen van deze techniek. Het hier beschreven werk, dat slechts een onderdeel vormt van het totale werk van de projectgroep, kon slechts uitgevoerd worden doordat het gebrek aan ervaring met Dopplertechnieken ruimschoots gecompenseerd werd met bezieling voor het werk en vertrouwen in de gekozen oplossingen. Daardoor was het mogelijk om een nieuwe benadering te kiezen. Kees Ruissen en Frans Smeets hebben een onmisbare rol gespeeld in het hele proces van orientatie, bezinning on experimenteren. zonder hun zou veel van wat nu bereikt is, onmogelijk zijn geweest. Ik bedank jullie beiden voor jullie bijdrage en hoop dat onze samenwerking zich verder zal ontplooien.

De basis voor het geheel werd gelegd door Rob Reneman, die reeds voor 1976 te maken had gehad met Dopplertoepassingen. Rob, je stond steeds klaar met je reeds verworven ervaring. Ondanks het feit dat de jekozen richting het voor een technische buitenstaander nagenoeg onmogelijk makt om de zaak tot in detail te volgen bleek je steeds in stat om de mogelijkheden ervan in te schatten en klaar te staan met nieuwe suggesties. Ik ben je dankbaar voor de mogelijkheden (o.a. de stage bij Pierre Peronneau in Parijs) en de steun die je me geboden hebt. Ik verheug me op een verdere en vruchtbare samenwerking.

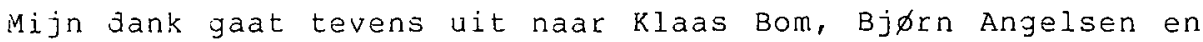
Jan Mol die met hun kritische kommentaren een waardevolle bijdrage geleverd hebben aan de uitvoering en de verslaglegging van dit promotiewerk.

Het vervaardigen van experimentele klinische diagnostische meetapparatuur is een zinloze zaak als er geen klinici vol on- 
geduld staan te wachten om deze apparatuur in de klinische praktijk uit te proberen en toe te passen. De door ons ontwikkelde apparatuur heeft zijn bestaansrecht gekregen door de manier warop Harm Bruins slot de mogelijkheden en beperkingen ervan buiten het laboratorium heeft weten te ontdekken. Dit gelat tevens voor Jan Mol, die vanaf het begin de ontwikkelingen gevolgd heeft.

In een later stadium wera de "Dopplergroep" geleidelijk aan uitgebreid met Huub Loete (transducerbouw en fysische experimenten), Tiny van Merode en Paul Hick (klinisch diagnostische metingen). Ik bedank hen voor hun bijdrage aan de ontwikkeling van meetmethodieken voor de diagnose van afwijkingen in bloedvaten. Mijn dank gat tevens uit nar Harry Peeters voor de enthousiaste manier warop hij de evaluatie (met behulp van een computermodel) van een nieuwe methode voor de detectie van de bloedsnelheid heeft uitgevoera.

Het zoeken naar nieuwe methoden kwam tevens tot uiting in de manier warop dit proefschrift gestalte heeft gekregen. Rosy Borgman, je wil om ae mogelijkheden van nieuwe apparatuur te ontdekken heeft ertoe geleid dat alles op een vloeiende en prettige manier is verlopen. 


\section{CURRICULUM VITAE}

Arnold Hoeks was born on November 16,1946 in Bergeyk, the Netherlands. From 1959 to 1964 he attended the Rythovius College in Eersel and from 1964 to 1971 the technical university of Eindhoven, department of electrical engineering. He concluded his study with a MSc thesis on a computer model on the interaction between thalamo-cortical relay cells and interneurons and the associated signal analysis at the Institute of Medical Physics, Utrecht, The Netherlands. He served as a Dutch peace corps volunteer from 1972 to 1974 in Iligan-City, Lanao del Norte, the Philippines, where he was assigned to the Mindanao state university - Iligan Institute of Technology as teacher in a 3-year experimental post-highschool technician training course. In 1975 he worked with the department of medical physics, Catholic University of Nijmegen, the Netherlands, where he participated in the development of a real-time spectral analyser to be used in the study of the auditory track of the cat and the grass-frog. From 1976 he was assigned to the department of biophysics, University of Limburg, Maastricht, the Netherlands, where he undertook a study on the development and applications of multi-gate pulsed Doppler systems suited for the investigation of the hemodynamic behaviour in peripheral arteries. 